\title{
Forward-backward correlations and charged-particle azimuthal distributions in $p p$ interactions using the ATLAS detector
}

\section{The ATLAS collaboration}

\begin{abstract}
Using inelastic proton-proton interactions at $\sqrt{s}=900 \mathrm{GeV}$ and $7 \mathrm{TeV}$, recorded by the ATLAS detector at the LHC, measurements have been made of the correlations between forward and backward charged-particle multiplicities and, for the first time, between forward and backward charged-particle summed transverse momentum. In addition, jet-like structure in the events is studied by means of azimuthal distributions of charged particles relative to the charged particle with highest transverse momentum in a selected kinematic region of the event. The results are compared with predictions from tunes of the PYTHIA and HERWIG++ Monte Carlo generators, which in most cases are found to provide a reasonable description of the data.
\end{abstract}

KEYwORDS: Hadron-Hadron Scattering 


\section{Contents}

1 Introduction 1

$\begin{array}{llr}2 & \text { Forward-backward correlations } & 3\end{array}$

3 Azimuthal distributions 4

4 Detector and event selection $\quad 6$

5 Monte Carlo models $\quad 6$

6 Analysis method $\quad 7$

6.1 Forward-backward correlations 8

$\begin{array}{lll}6.2 & \text { Azimuthal distributions } & 9\end{array}$

$\begin{array}{lll}7 & \text { Systematic uncertainties } & \mathbf{1 0}\end{array}$

$\begin{array}{lll}7.1 \text { Forward-backward correlations } & 10\end{array}$

$\begin{array}{ll}7.2 & \text { Azimuthal distributions } \\ \end{array}$

8 Results $\quad 11$

$\begin{array}{lll}8.1 & \text { Forward-backward correlations } & 11\end{array}$

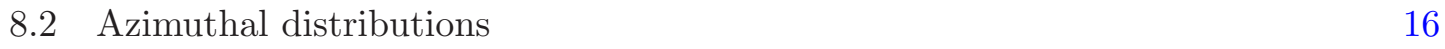

$\begin{array}{lll}9 & \text { Conclusions } & 17\end{array}$

$\begin{array}{ll}\text { A Summary of Monte Carlo tunes } & 21\end{array}$

The ATLAS collaboration $\quad 24$

\section{Introduction}

Two general classes of quantum chromodynamic processes combine to generate the events that are seen in high energy hadron collisions. The first of these comprises soft processes, which are commonly pictured in terms of multiple parton interactions at low transverse momentum, $p_{\mathrm{T}}$, modelled on multiple $t$-channel gluon exchanges. Processes of this kind, together with non-perturbatively described contributions such as the formation of beam remnants, are characterised by correlations between numbers of particles per unit of pseudorapidity over an extended pseudorapidity range [1]. At higher $p_{\mathrm{T}}$ values, harder processes arise that are dominated by perturbatively-described radiation or partonic scatters and are produced by single- and few-parton exchanges. These can generate particle jets, which are by definition characterised by short-range correlation; here high particle multiplicity is 
found within one unit in pseudorapidity, quickly diminishing as the pseudorapidity distance increases. Thus, soft processes produce lower particle multiplicities with weaker correlations over a wider pseudorapidity distance, so-called "long-range correlations", in comparison to the processes where a small number of jets are formed. As particle $p_{\mathrm{T}}$ values increase above a few hundred $\mathrm{MeV}$, there is a gradual transition between the soft regime and the hard. Hence, the correlation measured as a function of separation in pseudorapidity and of $p_{\mathrm{T}}$ probes the strength of each contribution.

A number of different event generators are available that combine the basic physical processes appropriately [2]; however, the more detailed aspects of the models need to be determined from data, a process referred to as "tuning" the models. For example, there are choices to be made as to whether the initial- and final-state gluon radiation are interleaved with the multiple parton interactions (MPI) and whether the modelling of the showers is to be ordered in terms of $p_{\mathrm{T}}$, angle, or the momentum transfer squared, $Q^{2}$, of the parton scatter. String or cluster fragmentation may be selected, and diffractive processes should be taken into consideration. Factors such as these can affect the correlations between the generated particles.

The tunings of the non-diffractive models have made use of output from many experiments; a variety of event characteristics were taken into account, such as distributions in particle multiplicity, transverse momentum and rapidity. A number of different tunes of the leading-order plus leading-logarithm generator PYTHIA [3-5] exist that provide a reasonably good description of events up to LHC energies [6-8]. The PYTHIA model uses string fragmentation $[9,10]$, in contrast to the cluster fragmentation used in the HERWIG model [11-13].

The correlations in pseudorapidity between the particles in an event have not been explicitly considered in the existing Monte Carlo (MC) tunes, and provide a further means of improving and helping to discriminate between the models [14]. Such correlations are the subject of the first part of the present study, which was carried out using proton-proton collisions recorded with the ATLAS detector [15] at the LHC. ${ }^{1}$ The measurements were inspired by earlier work by the UA5 experiment [16] and follow published studies at the LHC of the multiplicities themselves [17-21]. They also complement an earlier analysis by CMS of charged-particle correlations [22] and a recent analysis by ATLAS of two-particle correlations [23]. The approach taken here is to choose pairs of pseudorapidity intervals equal in size and symmetrically located in the forward and backward direction, allowing a measurement of what will be termed a forward-backward (FB) correlation between quantities in the two intervals. To determine the effects of both short-range and long-range physical correlations, the FB correlations are measured for increasing separation between the two selected pseudorapidity intervals.

\footnotetext{
${ }^{1}$ ATLAS uses a right-handed coordinate system with its origin at the nominal interaction point (IP) in the centre of the detector and the $z$-axis along the beam pipe. The $x$-axis points from the IP to the centre of the LHC ring, and the $y$-axis points upward. Cylindrical coordinates $(r, \phi)$ are used in the transverse plane, $\phi$ being the azimuthal angle around the beam pipe. The pseudorapidity is defined in terms of the polar angle $\theta$, with respect to the $z$-axis, as $\eta=-\ln \tan (\theta / 2)$. Throughout the paper the term "forward" is used to refer to the direction of positive $z$ or $\eta$, and "backward" to refer to the negative direction.
} 
Two types of FB correlation are presented: correlations in charged-particle multiplicity and correlations in the summed values of the transverse momenta of the charged particles. A further perspective comes from increasing the value of the minimum transverse momentum, $p_{\text {Tmin }}$, of the particles that are considered. In this way the effects of the soft processes can be gradually reduced, while the effects of the harder processes remain. The use of data taken at two very different proton-proton centre-of-mass energies, $\sqrt{s}=900 \mathrm{GeV}$ and $7 \mathrm{TeV}$, gives further information that can be compared with MC tunes based on $p \bar{p}$ interactions at the intermediate energy of the Tevatron, at $\sqrt{s}=1.96 \mathrm{TeV}$. The $7 \mathrm{TeV}$ data provide access to a new energy regime, which is the main goal of the LHC. It is important to understand the relationship between softer and harder processes in these two regimes in order to confirm our understanding of the background processes at the energies where new physics might be found.

Although such correlation measurements are sensitive to the presence of jets in events, no jet features are explicitly sought or exploited. Jets are by definition associated with short-range correlations in both pseudorapidity and azimuthal angle, and to obtain additional information about these correlations, a further analysis was performed using a different technique. Since the direction of a jet is often close to that of the highest- $p_{\mathrm{T}}$ particle in the event, the charged particle with the highest $p_{\mathrm{T}}$ is identified and the distributions of the other charged particles are studied as a function of their azimuthal angle relative to its direction.

The distribution of particles in the azimuthal plane of an event has been studied before at Tevatron energies [24-26] and at the LHC [27]. Taken relative to the highest$p_{\mathrm{T}}$ particle, the azimuthal distribution of particles shows two peaks, centred at zero and at $\pi$, that can indicate jet structure. In the events studied in the present measurement, however, these peaks are typically not observed in single events, most of which contain too few hard particles to form clearly defined jets, but become visible statistically when assembling the distribution over many events. The particle distributions also display the so-called "underlying event", the effects of which are particularly apparent at azimuthal angles perpendicular to the jets [27]. However, the principal goal of the present study is an understanding of the shape of the first jet-like peak, which is described differently depending on the MC model used. Again, it is found that the transition from $900 \mathrm{GeV}$ to $7 \mathrm{TeV}$ LHC energies provides a significantly different perspective on the physics.

This paper is structured as follows. The statistical methods for the correlation analysis and the azimuthal analysis are described in sections 2 and 3. Section 4 describes the ATLAS detector and the event selection, while the MC samples are described in section 5. Details of the analysis method are given in section 6 . The systematic uncertainties are discussed in section 7 , and the results and conclusions are presented in sections 8 and 9 .

\section{Forward-backward correlations}

All the correlations measured in this study, whether from symmetric or asymmetric pseudorapidity intervals, are referred to as forward-backward (FB) correlations and are measured using charged particles only. The FB multiplicity correlation, $\rho_{f b}^{n}$, between two particle 
multiplicities is the normalised covariance between the two distributions, relative to the mean value of each. For a given data sample or MC sample, it is defined in the usual way as:

$$
\rho_{f b}^{n}=\frac{\left\langle\left(n_{f}-\left\langle n_{f}\right\rangle\right)\left(n_{b}-\left\langle n_{b}\right\rangle\right)\right\rangle}{\sqrt{\left\langle\left(n_{f}-\left\langle n_{f}\right\rangle\right)^{2}\right\rangle\left\langle\left(n_{b}-\left\langle n_{b}\right\rangle\right)^{2}\right\rangle}}=\frac{\sum x_{f}^{n} x_{b}^{n}}{N \sigma_{f}^{n} \sigma_{b}^{n}} .
$$

Here, $n_{f}$ and $n_{b}$ are the respective multiplicities of particles of interest in two chosen forward and backward intervals in an event, \langle\rangle denotes a mean over the events in the sample, $N$ is the total number of events, $x_{f}^{n}, x_{b}^{n}$ are the differences between $n_{f}, n_{b}$ and their means, and $\sigma_{f}^{n}, \sigma_{b}^{n}$ are the standard deviations of $n_{f}$ and $n_{b}$ about their means. The sum in the right-hand side of the equation is taken over the events in the sample. In the present measurement, particles of interest are those above a given $p_{\text {Tmin }}$ value. Intervals in pseudorapidity of size $\delta \eta=0.5$ were chosen, which allows good statistical accuracy on each measured point while preserving a sensitivity to physically interesting variations with pseudorapidity $\eta$. A range $-2.5<\eta<2.5$ was considered, corresponding to the inner tracking detector acceptance, divided into five forward and five backward intervals.

The FB momentum correlation, $\rho_{f b}^{p_{\mathrm{T}}}$, between two summed transverse momentum $\left(\sum p_{\mathrm{T}}\right)$ values is defined similarly:

$$
\rho_{f b}^{p_{\mathrm{T}}}=\frac{\left\langle\left(\sum p_{\mathrm{T}}^{f}-\left\langle\sum p_{\mathrm{T}}^{f}\right\rangle\right)\left(\sum p_{\mathrm{T}}^{b}-\left\langle\sum p_{\mathrm{T}}^{b}\right\rangle\right)\right\rangle}{\sqrt{\left\langle\left(\sum p_{\mathrm{T}}^{f}-\left\langle\sum p_{\mathrm{T}}^{f}\right\rangle\right)^{2}\right\rangle\left\langle\left(\sum p_{\mathrm{T}}^{b}-\left\langle\sum p_{\mathrm{T}}^{b}\right\rangle\right)^{2}\right\rangle}}=\frac{\sum x_{f}^{p_{\mathrm{T}}} x_{b}^{p_{\mathrm{T}}}}{N \sigma_{f}^{p_{\mathrm{T}}} \sigma_{b}^{p_{\mathrm{T}}}},
$$

where $\sum p_{\mathrm{T}}^{f}$ and $\sum p_{\mathrm{T}}^{b}$ are the sums of the absolute transverse momentum values of the charged particles in the two chosen forward and backward intervals in an event, $x_{f}^{p_{\mathrm{T}}}, x_{b}^{p_{\mathrm{T}}}$ are the differences between $\sum p_{\mathrm{T}}^{f}, \sum p_{\mathrm{T}}^{b}$ and their means, and $\sigma_{f}^{p_{\mathrm{T}}}, \sigma_{b}^{p_{\mathrm{T}}}$ are the respective standard deviations. The $p_{\text {Tmin }}$ values used in measuring the correlations are discussed in the sections below.

\section{Azimuthal distributions}

To obtain sensitivity to the presence of jets and jet-like structures in the events, the highest$p_{\mathrm{T}}$ charged particle in a given $|\eta|$ region in an event is identified and termed the "leading particle". The azimuthal difference $\Delta \phi$ between the leading particle and any other accepted charged particle is the unsigned angle (in the range $0-\pi$ ) between the two particles in the transverse plane. The variable $N^{T}$ is defined as the number of selected charged particles, taken over the entire event sample, in a given interval of $\Delta \phi$ of width $\delta \phi$, where $\delta \phi$ takes the value $\pi / 50$ in the present analysis. A $p_{\mathrm{T}}>p_{\text {Tmin }}$ requirement is again imposed on the selected particles.

In the general case of jet production, it is expected that the distribution in $N^{T}$ should show peaks at $\Delta \phi=0$ and at $\Delta \phi=\pi$, with a minimum lying in between. As observed in the present study (figure 1a), this minimum takes the form of a flat contribution, or pedestal, which arises from the overall charged-particle activity in the event [27] together with uncorrelated backgrounds that are hard to model, such as fake tracks in the detector. The present study focuses on the peak structure, suppressing the influence of the pedestal 


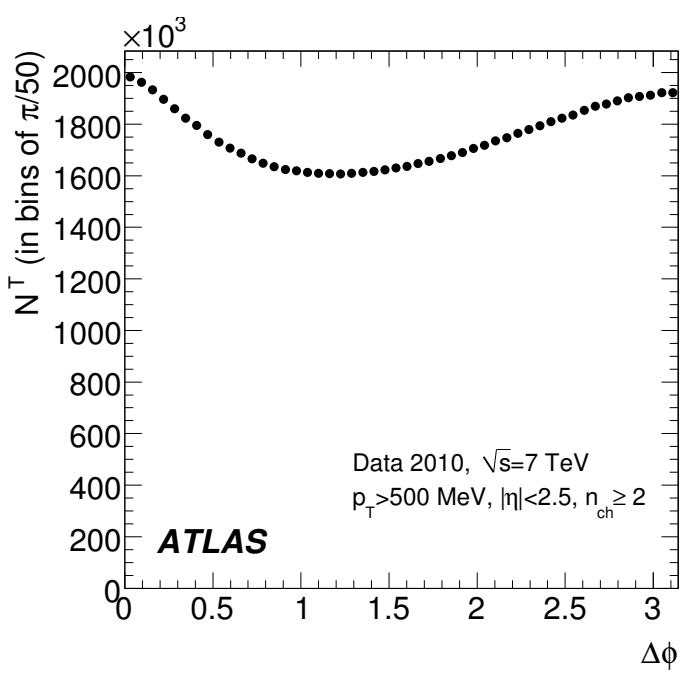

(a)

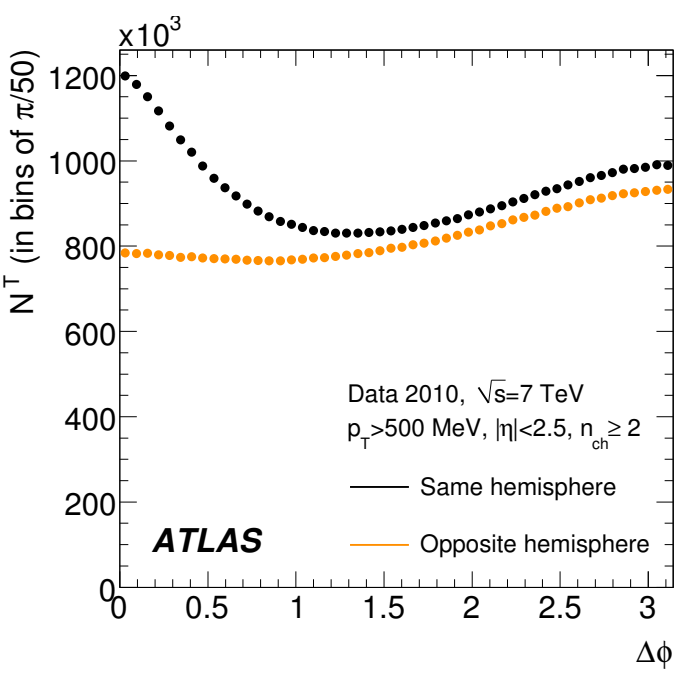

(b)

Figure 1. Azimuthal distributions for tracks with $p_{\text {Tmin }}=500 \mathrm{MeV}$ and $|\eta|<2.5$ in data at $\sqrt{s}=7 \mathrm{TeV}$. (a) Uncorrected distribution of $\Delta \phi$; (b) distributions for the same hemisphere in $\eta$ as the leading track, and for the opposite hemisphere. The statistical uncertainties are smaller than the plotted symbol.

on the measurement by performing a subtraction. Since the pedestal subtraction is performed in a corresponding way on the MC models tested, the latter do not need to model the pedestal level accurately for this analysis. In each case, we fit the observed $N^{T}$ distribution with a quadratic polynomial, determine the minimum value $N_{\min }^{T}$, and subtract this value from all the $N^{T}$ values in the distribution. The "subtracted" number of charged particles per radian in azimuthal angle is then normalised to give the quantity

$$
N_{\mathrm{sub}}^{T}=\frac{\left(N^{T}-N_{\min }^{T}\right)}{\sum\left(N^{T}-N_{\min }^{T}\right)},
$$

where the sum is taken over the bins of $\delta \phi$ used in the analysis.

Averaged over many events, the $N_{\text {sub }}^{T}$ variable is sensitive to the presence of jet pairs whenever both jets occur within the accepted $\eta$ interval. The direct effect of the second jet is removed, suppressing pedestal effects in an alternative way, by examining a second variable. We distinguish between particles produced in the same forward-backward event hemisphere in $\eta$ as the leading particle, and those produced in the opposite hemisphere $\left(\eta * \eta_{\text {lead }} \geq 0\right.$ and $<0$, respectively), denoting these as $N_{\text {same }}^{T}$ and $N_{\text {opp }}^{T}$ respectively, as shown in figure $1 \mathrm{~b}$. The normalised asymmetry is then defined as

$$
N_{\mathrm{SO}}^{T}=\frac{\left(N_{\text {same }}^{T}-N_{\mathrm{opp}}^{T}\right)}{\sum\left(N_{\text {same }}^{T}-N_{\mathrm{opp}}^{T}\right)} .
$$

On the assumption that the leading particle indicates the possible presence of a jet, $N_{\mathrm{SO}}^{T}$ is expected to take large positive values for $\Delta \phi \approx 0$ but to be smaller for larger $\Delta \phi$. Thus the jet structure associated with the leading particle is well-characterised, but the effects 
of the opposing jets tend on average to cancel out. Any non-zero value at large $\Delta \phi$ reflects long-range correlations in $\eta$ with jet effects largely removed. In this way, the two variables enhance the sensitivity to different aspects of jet-like events, and are sensitive to an aspect of long-range correlations that complements the FB measurements.

\section{Detector and event selection}

The ATLAS detector at the LHC covers almost the entire solid angle around the collision point with layers of tracking detectors, calorimeters and muon chambers. In the present analysis, tracks and vertices are reconstructed using the inner detector, which consists of a silicon pixel detector, a silicon strip detector and a transition radiation tracker. These extend in total from radii of $50.5 \mathrm{~mm}$ to $1066 \mathrm{~mm}$ from the beam line, and are located in a 2 tesla axial magnetic field provided by a superconducting solenoid. A track from a charged particle has typically 11 silicon detector hits and more than 30 transition radiation tracker hits. Coverage is obtained in the pseudorapidity range $|\eta|<2.5$.

The data analysed here were collected with the ATLAS detector in 2009 at $\sqrt{s}=900 \mathrm{GeV}$, and in early 2010 at $\sqrt{s}=7 \mathrm{TeV}$, corresponding to integrated luminosities of $7 \mu \mathrm{b}^{-1}$ and $190 \mu \mathrm{b}^{-1}$ respectively. The maximum instantaneous luminosity was $\sim 1.9 \times 10^{27} \mathrm{~cm}^{-2} \mathrm{~s}^{-1}$ (at $7 \mathrm{TeV}$ ), and the probability of additional interactions in the same bunch crossing as a given event was of the order of $10^{-3}$.

This analysis is based on the same dataset as was used for the ATLAS minimumbias particle multiplicity analysis [17] and uses the same minimum-bias scintillator trigger, the same event selections, and the same quality criteria on the track reconstruction and selection. An event in this dataset was required to have at least one primary vertex, formed from a minimum of two tracks with $p_{\mathrm{T}}>100 \mathrm{MeV}$ and consistent with the beamline. The primary vertex with tracks giving the highest $\Sigma p_{T}^{2}$ was selected, and events with a second primary vertex with four or more tracks were rejected, to minimise any possible effects due to pile-up. Tracks with an impact parameter above $1.5 \mathrm{~mm}$ in the transverse plane were removed to reduce the effects of particles from secondary decays. Around ten million events at $7 \mathrm{TeV}$ and one million events at $900 \mathrm{GeV}$ were selected at this stage.

For the FB correlation analysis, event samples were formed having at least two tracks in the event selected with $p_{\mathrm{T}}>p_{\text {Tmin }}$ and $|\eta|<2.5$, for a range of $p_{\text {Tmin }}$ values: 100, 200, $500 \mathrm{MeV}, 1.0,1.5,2.0 \mathrm{GeV}$ (see section 8.1). For the azimuthal analysis, a value of $p_{\text {Tmin }}=$ $500 \mathrm{MeV}$ was used in the final track and event selection, as discussed in section 6.2.

\section{Monte Carlo models}

It was necessary to apply corrections to the observed FB correlations in order to obtain the final results. These corrections were evaluated using simulated events generated with the MC09 tune of Pythia 6 [4, 28]. This tune, based on Pythia 6.4.21, was created by ATLAS to emulate the particle production at the Tevatron and provide predictions for the LHC. In PYTHIA 6, the initial-state gluon radiation is interleaved with the MPI, which is expected to reduce the difference in strength between short- and long-range correlations. 
Four further tunes, chosen to make use of different physical approaches as described below, were employed to evaluate model-dependent systematic effects. Around 800,000 MC events for each tune were used. Each of these samples contained a mixture of non-diffractive, single-diffractive and double-diffractive events. The subprocesses were combined according to the generator cross-sections. All the MC samples were passed through standard ATLAS software programs [29], based on GEANT4 [30], to simulate the trigger and detector effects before event reconstruction.

Two of the further tunes were also based on PYThia 6.4.21. The DW tune [31] was created to describe underlying-event (UE) data at the Tevatron and uses a $Q^{2}$-ordered shower, in contrast to the $p_{\mathrm{T}}$ shower ordering used by the other PYTHIA tunes. In the Perugia0 [32] tune the soft contribution was optimised using minimum-bias data from the Tevatron and the CERN Sp̄̄S colliders. A PYTHIA 8 sample [5] was also used, with default parameter values (Tune 1). In PYTHIA 8 , the final-state radiation (FSR) as well as the initial-state radiation (ISR) are interleaved with the MPI [33]. An alternative approach was provided by using PHOJET 1.12.3.15 [34]. This model uses a dual-parton event description, with soft hadronic processes described by pomeron exchange and semi-hard processes described by perturbative parton scattering. It has similar fragmentation and hadronisation to the PYTHIA model, and includes an additional central diffractive component.

The final results are compared with a selection of the above tunes and additional tunes, some of which have made use of LHC minimum-bias and underlying-event data. None of the tunes has taken account of FB correlation measurements. In general, the effect of the newer tunes has been to change the numbers of multiple parton interactions and the degree of colour reconnection. Perugia2011 [32] is a more recent tune of PYTHIA 6.4 that uses the usual $p_{\mathrm{T}^{-}}$ordered shower model, the interleaved MPI model, and a new set of colour reconnections. AMBT2B is a tune of PYTHIA 6.4 to ATLAS minimum-bias data [35]. The new Pythia $84 \mathrm{C}$ tune was used [5], and also HeRWIG++ [12, 13], in versions 2.1 and 2.5 , tuned to minimum-bias LHC data at $900 \mathrm{GeV}$ (tune MU900-2) and to the underlying event at $7 \mathrm{TeV}$ (tune UE7-2). The HERWIG model uses an angular-ordered parton shower and cluster fragmentation, but does not include diffractive processes, which constitute approximately $35 \%$ of the PYTHIA cross section and $20 \%$ of that of PHOJET. Appendix A presents in tabular form the main features of the PYTHIA and HERWIG tunes that have been used.

\section{Analysis method}

The correlations and distributions of physical interest are defined at the so-called "particle level" of the events; that is to say, in terms of the stable charged particles produced in the primary interaction with lifetime $c \tau>10 \mathrm{~mm}$. The kinematic region in a given measurement was defined by a requirement that there should be at least two such particles associated with the event, having $p_{\mathrm{T}}>p_{\text {Tmin }}$ and $|\eta|$ within a specified range up to a maximum value of 2.5. The FB correlations and the azimuthal distributions observed in the detector were corrected to the applicable kinematic region at the particle level. 


\subsection{Forward-backward correlations}

As observed in the detector, the FB correlations are modified by detector effects such as track reconstruction efficiency, trigger efficiency, vertex finding efficiency, and the detection of secondary particles arising from decays and interactions in the detector material. A simple weighting-factor correction to the number of measured tracks in a given interval (or to their summed $p_{\mathrm{T}}$ ) will not suffice when correlations are being measured, because the corrections may themselves be correlated between the different intervals. In the context of a set of variables with multiple correlations between them, a natural method to use for determining the correlations is multiple regression theory [36]. The following multiple regression technique was used to apply corrections to the observed FB correlations so as to obtain the FB correlations at the particle level.

In general, given four statistically varying quantities that may be correlated among themselves, the linear regression relationships take the form

$$
\begin{aligned}
& x_{1}=b_{12.34} x_{2}+b_{13.24} x_{3}+b_{14.23} x_{4} \\
& x_{2}=b_{21.34} x_{1}+b_{23.14} x_{3}+b_{24.13} x_{4} \\
& x_{3}=b_{31.24} x_{1}+b_{32.14} x_{2}+b_{34.12} x_{4} \\
& x_{4}=b_{41.23} x_{1}+b_{42.13} x_{2}+b_{43.12} x_{3} .
\end{aligned}
$$

Each variable $x_{i}$ denotes the difference between a statistically varying quantity and its sample mean. In the present case, this notation refers to track or charged-particle multiplicity, or to a summed transverse momentum value. In a given sample of events, each partial regression coefficient $b_{i j . k l}$ denotes how a chosen statistical quantity $x_{i}$ varies with one of the others, $x_{j}$, on average, for fixed values of the other two. A system of normal equations can be solved to determine these coefficients from the event sample.

The indices 1 and 2 are here used respectively to denote forward and backward particlelevel quantities, and the indices 3 and 4 to denote forward and backward observed quantities, as measured in the detector. Each quantity may be correlated with any of the others. Particularly strong partial correlations exist between $x_{1}$ and $x_{3}$, of course, and likewise between $x_{2}$ and $x_{4}$. The regression coefficients are determined by the use of MC event samples in which both the particle-level and the simulated detector-level quantities are known.

In order to link a FB correlation at the particle level $\rho_{\text {part }} \equiv \rho_{12}$ to the corresponding observed FB correlation $\rho_{\text {obs }} \equiv \rho_{34}$, we start from the second and third lines of (6.1). These equations are multiplied by $x_{3}$ and by $x_{2}$, respectively, and after using the definitions of equations (2.1) and (2.2) we obtain:

$$
\rho_{\mathrm{part}}=\alpha+\beta \cdot \rho_{\mathrm{obs}}
$$

where

$$
\alpha=\frac{b_{23.14} \sigma_{3}^{2}-b_{32.14} \sigma_{2}^{2}+b_{21.34} \sigma_{1} \sigma_{3} \rho_{13}-b_{34.12} \sigma_{2} \sigma_{4} \rho_{24}}{b_{31.24} \sigma_{1} \sigma_{2}}, \quad \beta=\frac{b_{24.13} \sigma_{3} \sigma_{4}}{b_{31.24} \sigma_{1} \sigma_{2}} .
$$

The particle-level correlations are therefore linearly related to the observed correlations and may be obtained from them by means of the two coefficients $\alpha$ and $\beta$. 
The relationships between $x_{1}$ and $x_{3}$, and between $x_{2}$ and $x_{4}$, are due largely to the effect of track reconstruction efficiency; these relationships are contained in the coefficient $\beta$. The other terms contain detector effects such as trigger and vertex finding efficiencies, and also effects of physical FB correlations, as modelled in the MC simulations. These affect the coefficient $\alpha$, where they partially cancel. The values of $\alpha$ and $\beta$ were calculated using a range of $\mathrm{MC}$ tunes together with the detector simulation, and the variations on the values obtained then give rise to a systematic uncertainty on the results. This uncertainty has been reduced to a low level by this method and is contained mainly within the coefficient $\alpha$, whose value was found to lie in the range $0.07 \pm 0.03$. The value of $\beta$ lies in the range $0.96-$ $0.97(0.97-0.98)$ for the multiplicity ( $\operatorname{summed} p_{\mathrm{T}}$ ) measurements. The full set of systematic uncertainties on the results is discussed in section 7 .

To validate the above approach, the linearity of the relationship 6.2 was checked for both $\mathrm{MC}$ and data. It was verified from the MC samples that the analysis procedure regenerated the correlations that were calculated from the generated particles directly. The data sample did not include events produced with fewer than two reconstructed tracks associated with the primary vertex; a correction of up to $0.9 \%$ was applied for the small bias associated with this.

\subsection{Azimuthal distributions}

The azimuthal analysis employed the same track and event selection as the FB correlations analysis (section 4) but imposed a higher minimum transverse momentum requirement of $p_{\text {Tmin }}=500 \mathrm{MeV}$ on accepted tracks. This minimises systematic uncertainties by selecting a kinematic region with track reconstruction efficiencies that are flat in $p_{\mathrm{T}}$, a procedure made necessary by the subtraction methods used in this section of the analysis. Histograms of $N_{\text {sub }}^{T}$ and $N_{\text {SO }}^{T}$ were made as a function of $\Delta \phi$, and required corrections for detector effects in order to obtain the particle-level distributions.

One set of effects comes from a loss of non-leading tracks due to detector inefficiency, and contamination from non-primary tracks. To correct for these effects, each non-leading track was weighted by a factor $(1-b) / \epsilon$, where $b$ represents the fraction of background due to non-primary tracks and $\epsilon$ is the mean track reconstruction efficiency for the selected tracks, evaluated as a function of $p_{\mathrm{T}}$ and $\eta$. These terms were evaluated from MC simulation. The normalisation procedure gives a large cancellation of the effects of non-leading track reconstruction inefficiency, while preserving the shape of the variations in $\epsilon$; these are more important than the average value.

The loss of a leading track, due to track reconstruction inefficiency, may lead to the loss of the event or else to the wrong identification of a non-leading track as the leading track. A correction was applied to the observed distributions of figure 1 to remove the shape distortions caused by these effects. It was done in three steps. First, in a given $p_{\mathrm{T}}$ and $\eta$ range, the fraction $\epsilon_{l}$ of events that have the correct leading track was estimated by integrating the track reconstruction efficiency (determined from MC-simulated events) over the observed $p_{\mathrm{T}}$ and $\eta$ spectrum of the events in the data. Second, starting from the observed data distribution in $\Delta \phi$, a series of new distributions was constructed by removing an increasingly large random fraction $f$ of the observed leading tracks from the 
data sample. The resulting shape distortion was found to be linear in $f$ in each $\Delta \phi$ bin. This made it possible to extrapolate the original data distribution to what it would have been if $\epsilon_{l}$ were $100 \%$. The correction to the final results is up to $2 \%$.

The distributions of $N_{\text {sub }}^{T}$ and $N_{\text {SO }}^{T}$ were made for three ranges of $\eta$, namely $|\eta|<1.0$, 2.0 and 2.5 , in order to provide a broad-based comparison with the MC models.

\section{Systematic uncertainties}

\subsection{Forward-backward correlations}

The largest systematic uncertainty on the results arises from the uncertainty on the track reconstruction efficiency. A smaller effect arises from the variation of the correction parameters $\alpha$ and $\beta$ with the MC model used to obtain them (cf. section 6.1). The effects from the uncertainties on trigger and vertex finding efficiencies are small.

In this section, uncertainties are given as percentages of the central values and symmetric positive and negative uncertainties are taken. The model-dependence of the analysis was evaluated for the $7 \mathrm{TeV}$ data, and the conclusions were also applied to the $900 \mathrm{GeV}$ data. The chosen initial set of five MC tune samples were used to correct the data, and the spread in the results relative to the standard MC09, of the order of $1 \%$, was taken as the systematic uncertainty due to the model-dependence of the correction procedure. In addition, the diffractive components of the MC09 sample were varied by $\pm 10 \%$; this changed the measured correlations by typically $\pm 0.8 \%$ of their value.

The systematic effects on the FB correlations due to uncertainties on the trigger, vertex finding and track reconstruction efficiencies were studied by varying these efficiencies in the $\mathrm{MC}$, and then re-evaluating the FB correlations using the standard correction parameters. Uncertainties on efficiencies of $0.9 \%(1.6 \%)$ and $0.4 \%(0.6 \%)$ were used at $7 \mathrm{TeV}(900 \mathrm{GeV})$ for the trigger and vertex finding, respectively, and a track reconstruction uncertainty varying between $2 \%$ and $15 \%$ [17] was used. The systematic variations on the standard selection were achieved by removing a fraction of the events or tracks in a sample, randomly selected, according to the uncertainties on the respective efficiencies. This simulated a decrease in the efficiency which was taken as the corresponding systematic uncertainty.

In the case of the trigger and vertex efficiencies, the deviations on the FB correlations are $\leq 0.2 \%$ and $\sim 0.2 \%$, respectively. When the number of tracks in the events was varied as described above, the resulting FB correlation varied by $1.2 \%(1.4 \%)$ in the central pair of $\eta$-intervals, increasing to $3.3 \%(3.0 \%)$ in the outermost pair for multiplicity (momentum).

The total systematic uncertainty was obtained by summing the individual contributions in quadrature, and was combined in quadrature with the statistical uncertainty to obtain the total uncertainty. The total systematic uncertainty is dominated by the errors on the model-dependence and on the track reconstruction efficiency, and both of these are highly correlated between the two energies. In taking the ratios of the correlations at different energies, the systematic uncertainties are therefore small relative to the statistical and can be neglected. 


\subsection{Azimuthal distributions}

Performing the subtractions and the normalisations reduces the systematic uncertainties on the $\Delta \phi$ distributions considerably. Of primary importance is the uncertainty on the shape of the distribution, quantified here by estimating the relative uncertainty on the first bin in $\Delta \phi$. The most important effect comes from the event selection efficiency ( $1 \%$ in the $900 \mathrm{GeV}$ data and $3 \%$ in the $7 \mathrm{TeV}$ data), and mainly affects events with a small number of tracks. Other important effects come from the correction procedure, which contributes $2 \%$, and the effects of the track momentum resolution in the vicinity of the boundaries of the kinematic acceptance. The latter was estimated by varying the values of the cuts on $p_{\mathrm{T}}$ and $\eta$ by their resolution and repeating the analysis. This gave variations of $1-2 \%$ and $0.2-0.5 \%$ from $p_{\mathrm{T}}$ and $\eta$ respectively.

A variety of additional effects gave uncertainties at the $0.1-0.3 \%$ level. These comprised errors in the choice of leading charged particle (estimated from MC), uncertainty on the fraction of leading particles that were not reconstructed (also estimated from MC), and uncertainties on the track reconstruction efficiencies of the non-leading tracks. The uncertainty on the track reconstruction efficiency, measured to be on average 15\% [17], was propagated to the non-leading track correction. It corresponds to a relative systematic uncertainty on the distribution of $0.1-0.2 \%$. A final contribution of similar magnitude came from uncertainties on the number of secondary particles, depending on the choice of impact parameter cut. The track reconstruction efficiency difference between the forward and backward halves of the detector was found to have a negligible effect.

The total systematic uncertainty was obtained by summing the individual contributions in quadrature, and was combined in quadrature with the statistical uncertainty to obtain the total uncertainty. The statistical uncertainties dominate the measurements at $900 \mathrm{GeV}$, but in the $7 \mathrm{TeV}$ data the statistical and systematic uncertainties are comparable.

\section{Results}

\subsection{Forward-backward correlations}

The observed FB correlations were corrected to the particle level, using the procedure described in section 6 , and all results are quoted at this level. The correction typically increased the correlation value above the observed, uncorrected value by $0.05-0.08$, in both multiplicity and momentum.

To obtain an overall picture, and as a check on the experimental approach, the FB multiplicity correlations using $7 \mathrm{TeV}$ collisions were calculated over a matrix of forwardbackward pairs of $\eta$ intervals of width $\delta \eta=0.5$ covering the range 0 to 2.5 in each direction, for a $p_{\text {Tmin }}$ value of $100 \mathrm{MeV}$. The results are given in table 1 and shown pictorially in figure 2. The main diagonal depicts the symmetric FB correlations. Both for the data and for the chosen MC, the correlations are seen to vary strongly with the separation between the chosen intervals, and only weakly with the mean $\eta$ value for a given separation, confirming that the symmetric forward-backward configuration is a characteristic representation of the correlation at a given separation in $\eta$. 


\begin{tabular}{|c|c|c|c|c|c|}
\hline $\begin{array}{c}\text { Forward } \eta \text { interval } \\
\text { Backward } \eta \text { interval }\end{array}$ & $0.0-0.5$ & $0.5-1.0$ & $1.0-1.5$ & $1.5-2.0$ & $2.0-2.5$ \\
\hline $0.0-0.5$ & 0.666 & 0.624 & 0.592 & 0.566 & 0.540 \\
& \pm 0.011 & \pm 0.011 & \pm 0.011 & \pm 0.012 & \pm 0.013 \\
\hline $0.5-1.0$ & 0.624 & 0.596 & 0.574 & 0.553 & 0.530 \\
& \pm 0.011 & \pm 0.011 & \pm 0.012 & \pm 0.013 & \pm 0.014 \\
\hline $1.0-1.5$ & 0.594 & 0.576 & 0.560 & 0.540 & 0.518 \\
& \pm 0.011 & \pm 0.012 & \pm 0.013 & \pm 0.014 & \pm 0.014 \\
\hline $1.5-2.0$ & 0.571 & 0.557 & 0.544 & 0.526 & 0.503 \\
& \pm 0.012 & \pm 0.013 & \pm 0.014 & \pm 0.014 & \pm 0.016 \\
\hline $2.0-2.5$ & 0.551 & 0.540 & 0.527 & 0.507 & 0.487 \\
& \pm 0.013 & \pm 0.014 & \pm 0.014 & \pm 0.016 & \pm 0.018 \\
\hline
\end{tabular}

Table 1. Multiplicity correlations for events at $\sqrt{s}=7 \mathrm{TeV}$ for events with a minimum of two charged particles in the kinematic interval $p_{\mathrm{T}}>100 \mathrm{MeV}$ and $|\eta|<2.5$ for different combinations of forward and backward pseudorapidity interval. The quoted uncertainties are the systematic uncertainties; the statistical uncertainties are approximately \pm 0.001 or less and are small by comparison.

In the remainder of this section, the correlations between symmetrically opposite FB pseudorapidity intervals of width $\delta \eta=0.5$ are examined. A global correlation has also been calculated, defined as the FB correlation taken over the entire pseudorapidity range $(\delta \eta=2.5)$. The results are summarised in table 2. Figure 3(a) shows the variation of the FB multiplicity correlation with $\eta$ found in the $7 \mathrm{TeV}$ data, compared with various MC tunes. Figure 3(b) shows the same quantities measured at $900 \mathrm{GeV}$. The general shape of the data distributions is reasonably well reproduced by the MCs, though there are discrepancies in detail. In the case of MC09, the trend is to underestimate the correlation by $\sim 10 \%$. The correlation is generally similar for the DW and MC09 tunes at $7 \mathrm{TeV}$, but shows greater variation at $900 \mathrm{GeV}$ where it rises relative to the data at high $\eta$ values, implying an inaccuracy in the modelled ratio of long-distance to short-distance correlations. The Pyтнia 8 tune performs well at low $\eta$ but somewhat poorly at higher values. The correlations given by AMBT2B are the most consistent with the data; AMBT2B was tuned to these event samples using different variables. At both energies HERWIG ++ gives correlations consistently higher than the data.

Figure 3(c) shows the ratio of the FB multiplicity correlation distributions at $900 \mathrm{GeV}$ and $7 \mathrm{TeV}$, compared with the results from the MC tunes. The multiplicity correlations are substantially lower at $900 \mathrm{GeV}$ than at $7 \mathrm{TeV}$ and the relative difference is greater for the larger pseudorapidity bins. This is a new experimental result which can be interpreted in general terms as indicating that, while the short-range correlation is similar at the two energies, the long-range correlation becomes considerably higher as the energy increases. This trend is reproduced in most of the MC predictions, although not so well by DW. The HERWIG ++ ratio is not included since different tunes are used at the two energies. 


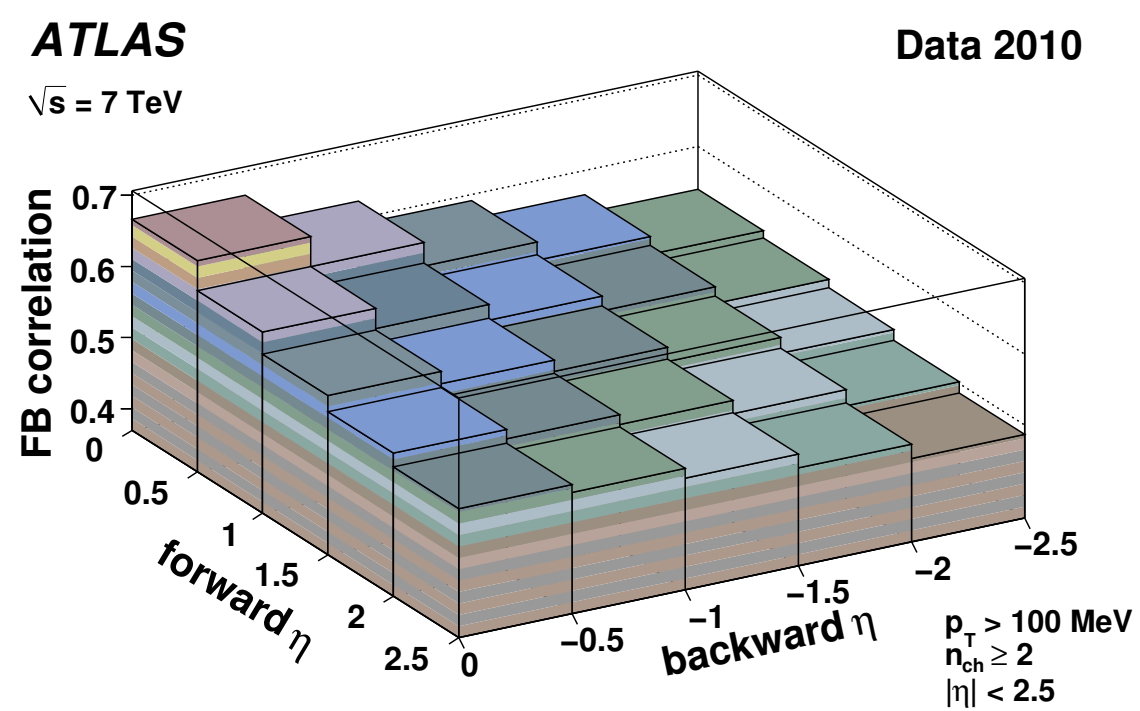

(a)

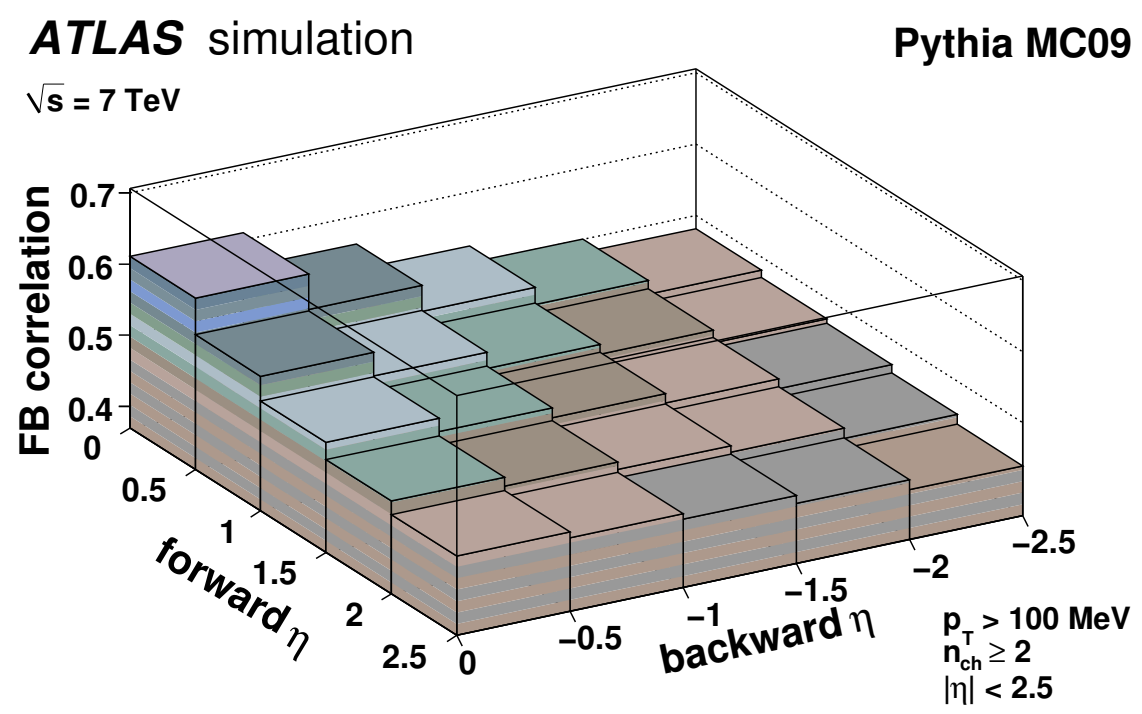

(b)

Figure 2. Multiplicity correlation at $7 \mathrm{TeV}$ in a matrix of forward/backward $\eta$-intervals for $|\eta|<$ 2.5 , for events with at least two charged particles with $p_{\mathrm{T}}>100 \mathrm{MeV}$ and $|\eta|<2.5$. (a) data; (b) PYTHia 6 MC09 tune. The statistical uncertainties are of the order of \pm 0.001 .

The minimum value, $p_{\text {Tmin }}$, of the transverse momentum of the selected charged particles was varied for the $7 \mathrm{TeV}$ data. The resulting multiplicity correlations are shown in table 3 and illustrated in figure 4 . As expected, the correlations fall rapidly as $p_{\text {Tmin }}$ increases above a few hundred $\mathrm{MeV}$, a feature also seen in the $\mathrm{MC}$ models (not shown). In the context of the Lund string model [9], at low transverse momentum values, this may be seen as a general tendency for a partonic string to fragment in a uniform way all along its length. There is also a possible influence of MPI effects. At higher transverse momentum, however, particles are more likely to be associated with jets, and there is no strong corre- 


\begin{tabular}{|c|c|c|c|c|c||c|}
\hline$|\eta|$ interval & $0.0-0.5$ & $0.5-1.0$ & $1.0-1.5$ & $1.5-2.0$ & $2.0-2.5$ & $0.0-2.5$ \\
\hline $900 \mathrm{GeV}$ & 0.498 & 0.407 & 0.359 & 0.310 & 0.259 \\
& $\pm 0.001 \pm 0.011$ & $\pm 0.001 \pm 0.011$ & $\pm 0.002 \pm 0.013$ & $\pm 0.002 \pm 0.015$ & $\pm 0.002 \pm 0.015$ & $\begin{array}{c}0.652 \\
\pm 0.001 \pm 0.007 \\
\end{array} 7 \mathrm{TeV}$ \\
& 0.666 & 0.596 & 0.560 & 0.526 & 0.487 & 0.801 \\
& \pm 0.011 & \pm 0.011 & \pm 0.013 & \pm 0.014 & \pm 0.018 & \pm 0.008 \\
\hline
\end{tabular}

Table 2. Forward-backward multiplicity correlation for charged particles in symmetrically opposite $\eta$-intervals for events with a minimum of two charged particles in the kinematic interval $p_{\mathrm{T}}>$ $100 \mathrm{MeV}$ and $|\eta|<2.5$ at $\sqrt{s}=900 \mathrm{GeV}$ and $7 \mathrm{TeV}$. The final column shows the global result. The quoted uncertainties are first statistical, second systematic; where the statistical uncertainty is less than approximately \pm 0.001 , only the systematic uncertainty is shown.

\begin{tabular}{|c|c|c|c|c|c||c|}
\hline $\begin{array}{c}|\eta| \text { interval } \\
p_{\text {Tmin }}[\mathrm{GeV}]\end{array}$ & $0.0-0.5$ & $0.5-1.0$ & $1.0-1.5$ & $1.5-2.0$ & $2.0-2.5$ & $0.0-2.5$ \\
\hline 0.1 & 0.666 & 0.596 & 0.560 & 0.526 & 0.487 & 0.801 \\
& \pm 0.011 & \pm 0.011 & \pm 0.013 & \pm 0.014 & \pm .018 & \pm 0.008 \\
\hline 0.2 & 0.639 & 0.565 & 0.534 & 0.498 & 0.458 & 0.790 \\
& \pm 0.009 & \pm 0.010 & \pm 0.011 & \pm 0.012 & \pm 0.017 & \pm 0.008 \\
\hline 0.3 & 0.602 & 0.529 & 0.500 & 0.462 & 0.426 & 0.775 \\
& \pm 0.009 & \pm 0.009 & \pm 0.011 & \pm 0.012 & \pm 0.016 & \pm 0.008 \\
\hline 0.5 & 0.518 & 0.445 & 0.416 & 0.377 & 0.341 & 0.726 \\
& \pm 0.009 & \pm 0.009 & \pm 0.010 & \pm 0.014 & \pm 0.017 & \pm 0.010 \\
\hline 1.0 & 0.291 & 0.216 & 0.190 & 0.158 & 0.132 & 0.506 \\
& \pm 0.010 & \pm 0.011 & \pm 0.013 & \pm 0.018 & \pm 0.017 & \pm 0.023 \\
\hline 1.5 & 0.128 & 0.063 & 0.047 & 0.026 & 0.010 & 0.234 \\
& $\pm 0.001 \pm 0.006 \pm 0.001 \pm 0.008$ & $\pm 0.001 \pm 0.016$ & $\pm 0.001 \pm 0.012$ & $\pm 0.001 \pm 0.014$ & \pm 0.033 \\
\hline 2.0 & 0.036 & -0.020 & -0.031 & -0.041 & -0.046 & -0.008 \\
& $\pm 0.001 \pm 0.006$ & $\pm 0.001 \pm 0.008$ & $\pm 0.001 \pm 0.007$ & $\pm 0.001 \pm 0.005$ & $\pm 0.001 \pm 0.007$ & $\pm 0.001 \pm 0.019$ \\
\hline
\end{tabular}

Table 3. Forward-backward charged-particle multiplicity correlations at $\sqrt{s}=7 \mathrm{TeV}$, in symmetrically opposite $\eta$-intervals, for events with at least two charged particles with $|\eta|<2.5$ and $p_{T}>p_{\text {Tmin }}$, for varying values of $p_{\text {Tmin }}$. The final column shows the global result. The quoted uncertainties are first statistical, second systematic; where the statistical uncertainty is less than approximately \pm 0.001 , only the systematic uncertainty is shown.

lation between a given jet and another jet at any particular value of $\eta$. The tendency to have negative correlation values at higher $p_{\mathrm{T}}$ is a manifestation of the tendency of high- $p_{\mathrm{T}}$ particles to be collimated into jets.

The FB correlations between the summed $p_{\mathrm{T}}$ of charged particles are shown in figure 5 and listed in table 4. In general, the features are similar to those of the multiplicity correlations. As in the case of the multiplicities, the momentum FB correlations are higher at $7 \mathrm{TeV}$ than at $900 \mathrm{GeV}$. 


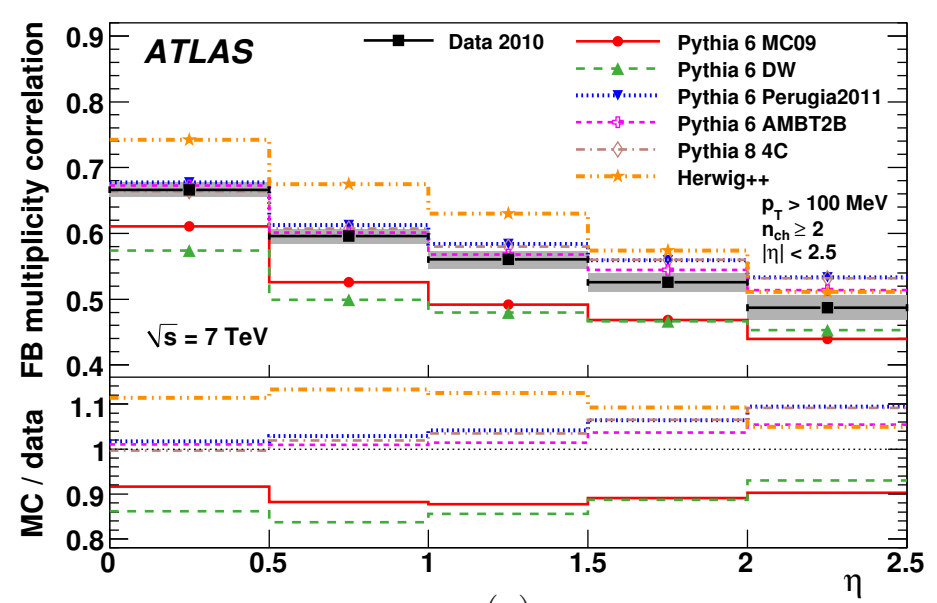

(a)
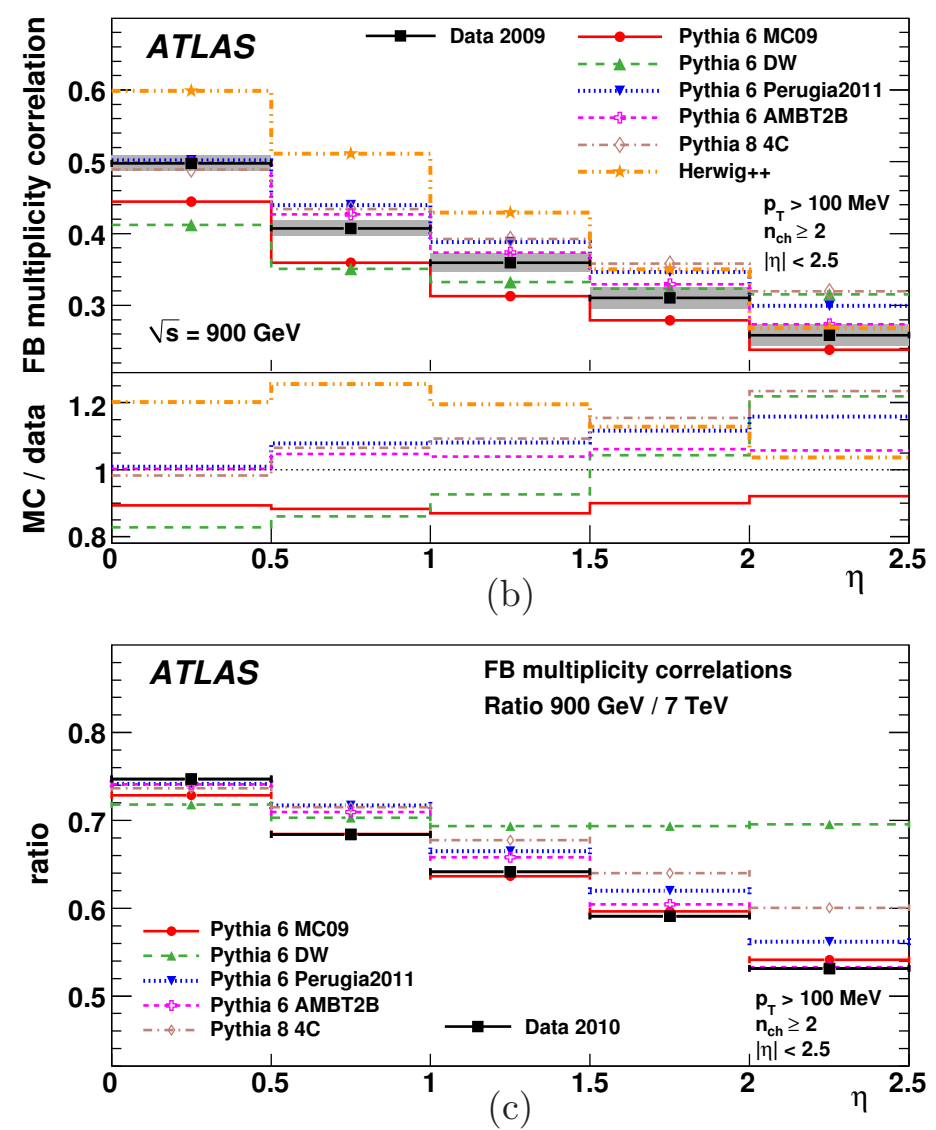

Figure 3. Forward-backward multiplicity correlation in symmetrically opposite $\eta$ intervals for events with at least two charged particles with $p_{\mathrm{T}} \geq 100 \mathrm{MeV}$ and $|\eta|<2.5$. (a) Data at $7 \mathrm{TeV}$, compared with a selection of MC simulations. The systematic uncertainties are indicated by a grey band; the statistical uncertainties are too small to be visible on the figure. (b) The same at $900 \mathrm{GeV}$. (c) Ratio of the $900 \mathrm{GeV}$ results to the $7 \mathrm{TeV}$ results. 


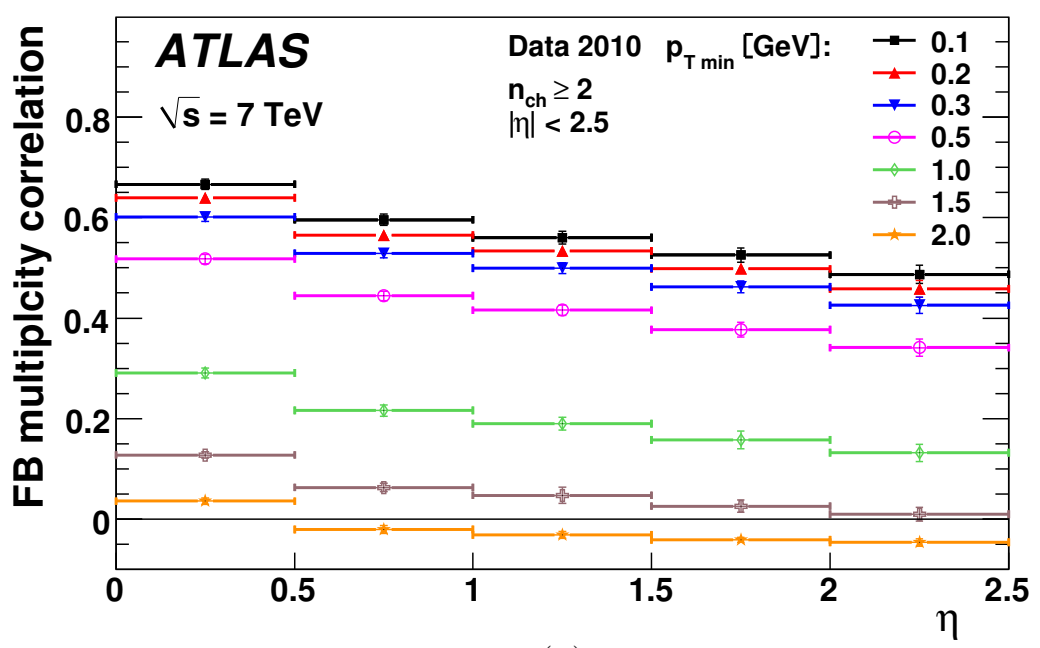

(a)

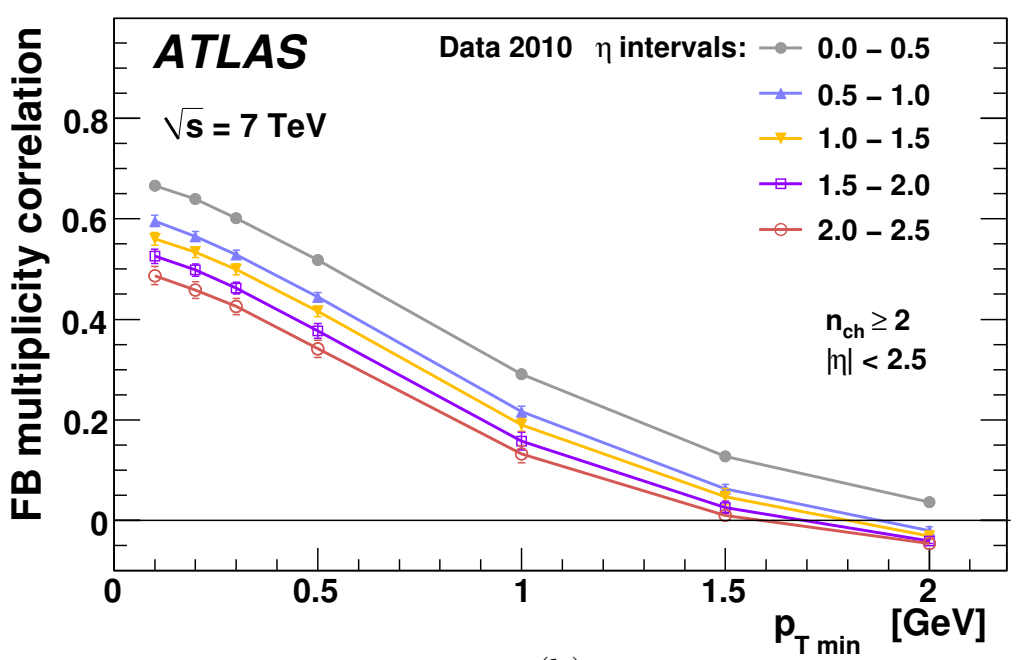

(b)

Figure 4. Forward-backward multiplicity correlations: (a) as a function of $\eta$ for different values of $p_{\text {Tmin }}$; (b) as a function of $p_{\text {Tmin }}$ for different values of $\eta$, with lines drawn to guide the eye. The plotted systematic uncertainties are smaller than the symbol in many cases; the statistical uncertainties are too small to be visible on the figure.

\subsection{Azimuthal distributions}

Distributions of the subtracted and the "same hemisphere minus opposite" charged-particle distributions in azimuthal angle, $N_{\text {sub }}^{T}$ and $N_{\text {SO }}^{T}$, are shown in figures 6 and 7 , respectively, for $\sqrt{s}=900 \mathrm{GeV}$ and $7 \mathrm{TeV}$, and compared with a selection of the different MC tunes. The leading particle in each event is omitted from the plots. One notes in figure 6 the increasing prominence of the "opposite" jet peak in the distributions as the $|\eta|$ interval increases, as more pairs of jets, separated in $\eta$, become included within the acceptance. The shapes show a strong energy dependence. 


\begin{tabular}{|l|c|c|c|c|c||c|}
\hline$|\eta|$ interval & $0.0-0.5$ & $0.5-1.0$ & $1.0-1.5$ & $1.5-2.0$ & $2.0-2.5$ & $0.0-2.5$ \\
\hline $900 \mathrm{GeV}$ & 0.445 & 0.362 & 0.328 & 0.284 & 0.236 & 0.637 \\
& $\pm 0.001 \pm 0.008$ & $\pm 0.002 \pm 0.009$ & $\pm 0.002 \pm 0.010$ & $\pm 0.002 \pm 0.013$ & $\pm 0.003 \pm 0.009$ & $\pm 0.001 \pm 0.009$ \\
\hline $7 \mathrm{TeV}$ & 0.590 & 0.519 & 0.492 & 0.455 & 0.412 & 0.770 \\
& \pm 0.010 & \pm 0.009 & \pm 0.010 & \pm 0.011 & \pm 0.016 & ${ }_{-0.008}^{+0.007}$ \\
\hline
\end{tabular}

Table 4. Forward-backward summed $p_{\mathrm{T}}$ correlation for charged particles in symmetrically opposite $\eta$ intervals, for events with at least two charged particles in the kinematic interval $p_{\mathrm{T}}>100 \mathrm{MeV}$ and $|\eta|<2.5$ at $\sqrt{s}=900 \mathrm{GeV}$ and $7 \mathrm{TeV}$, for corrected data. The quoted uncertainties are first statistical, second systematic; where the statistical uncertainty less than approximately \pm 0.001 , only the systematic uncertainty is shown.

In general, the MC tunes and models used for comparison with data provide a reasonable description in the restricted rapidity range $|\eta|<1$, but the agreement becomes increasingly poor as the $|\eta|$ range is increased to 2 and 2.5. For the subtracted distributions the Perugia2011 tune is the most successful, giving a good representation of the distribution at $900 \mathrm{GeV}$ for all three $|\eta|$ ranges. At $7 \mathrm{TeV}$ it is poorer for $|\eta|<1$, but similar to the other tunes and models presented, and it improves as the coverage in $|\eta|$ increases. The other three models are very similar at $900 \mathrm{GeV}$, where they all give an equally poor description of the data in the broader $|\eta|$ ranges. At $7 \mathrm{TeV}$, DW describes the data well for $|\eta|<1$, but is a poorer match in the higher $|\eta|$ ranges. HERWIG ++ and PYTHIA 8 are similar at all $|\eta|$, giving a somewhat poor description that resembles Perugia2011 at $|\eta|<1$ and DW at higher $|\eta|$. In all cases, the discrepancies of the tunes and models tend towards over-enhancing the same-side peak relative to the opposite-side peak.

The same-minus-opposite variable is described well for $|\eta|<1$ at both energies by PYTHIA 8, Perugia2011 and HERWIG++, with regard to both the leading-particle "jet peak" and the opposing pedestal. However DW exaggerates the sharpness of the peak. As the accepted range in $|\eta|$ increases, so that the averaged effects of more widely-separated jet pairs become included in the distributions, the disagreements become more pronounced. At both energies DW's exaggeration of the peak increases dramatically with $|\eta|$, while PYTHIA 8 gives a progressively worse description of the peak, also overestimating its size. The Herwig ++ and Perugia2011 models are very similar in this variable, and describe the data well at $900 \mathrm{GeV}$ but less so at $7 \mathrm{TeV}$, where they also resemble PyтнiA 8. Again, the discrepancies tend towards an over-enhancement of the same-side "jet peak", which becomes a particularly strong effect in the case of DW.

\section{Conclusions}

The forward-backward correlation in charged-particle multiplicity and in summed chargedparticle $p_{\mathrm{T}}$ has been measured at ATLAS in minimum-bias events at $\sqrt{s}=900 \mathrm{GeV}$ and $7 \mathrm{TeV}$. This is the first measurement of summed- $p_{\mathrm{T}}$ correlations. Both types of correlation fall in strength with increasing separation in pseudorapidity. The multiplicity correlation is found to differ by up to about $15 \%$ from some of the standard Monte Carlo tunes at 


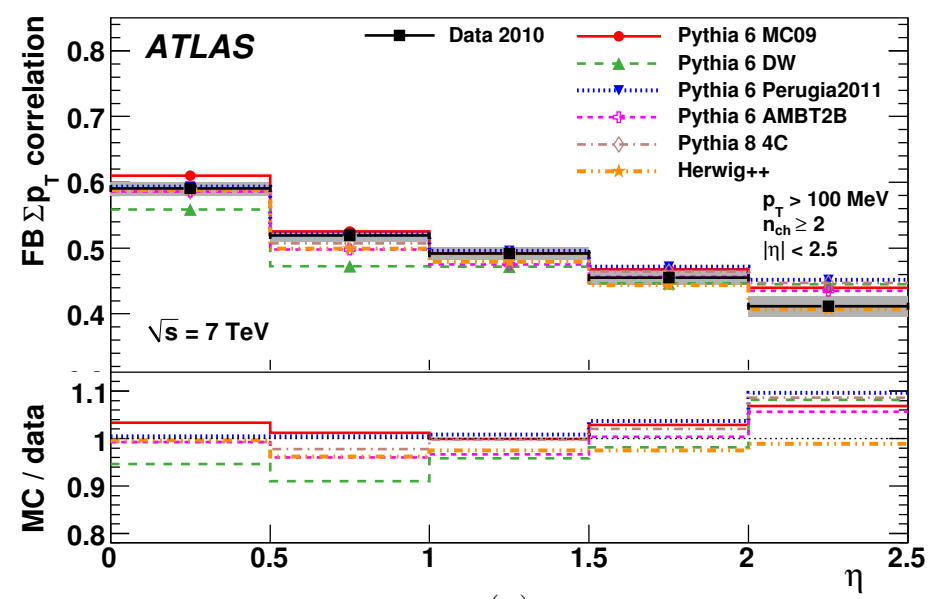

(a)

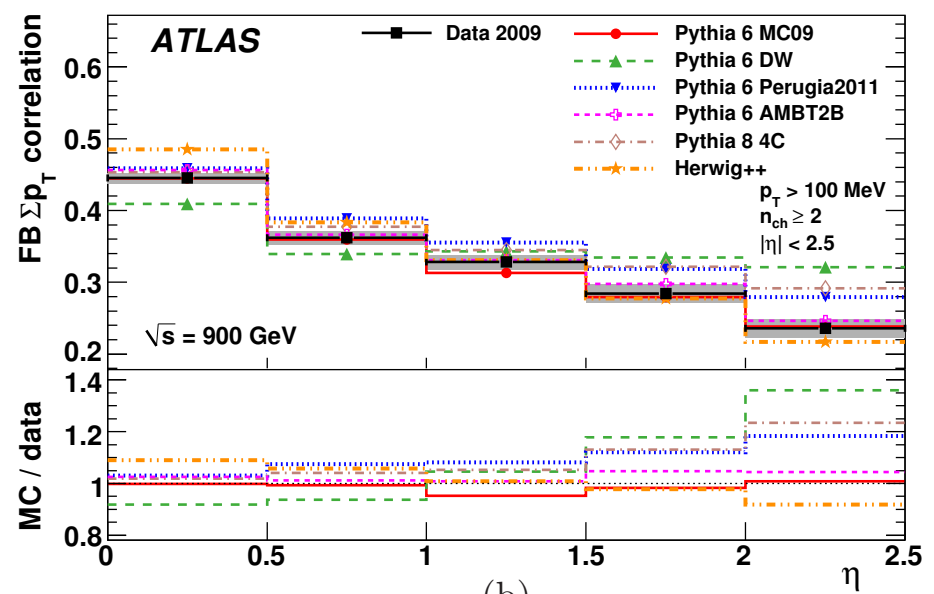

(b)

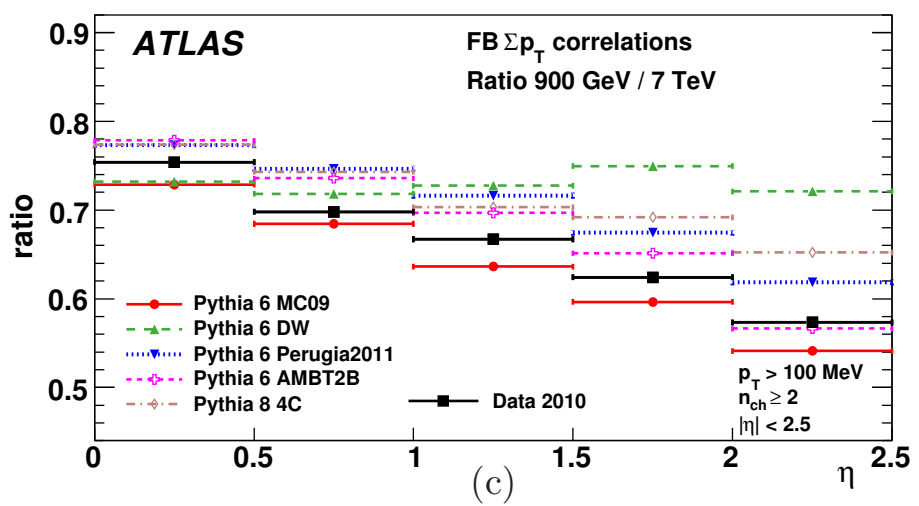

Figure 5. Forward-backward $\Sigma p_{\mathrm{T}}$ correlation in symmetrically opposite $\eta$ intervals for events with at least two charged particles with $p_{\mathrm{T}} \geq 100 \mathrm{MeV}$ and $|\eta|<2.5$. (a) Data at $7 \mathrm{TeV}$, compared with a selection of MC simulations. The systematic uncertainties are indicated by a grey band; the statistical uncertainties are too small to be visible on the figure. (b) The same at $900 \mathrm{GeV}$. (c) Ratio of the $900 \mathrm{GeV}$ results to the $7 \mathrm{TeV}$ results. 


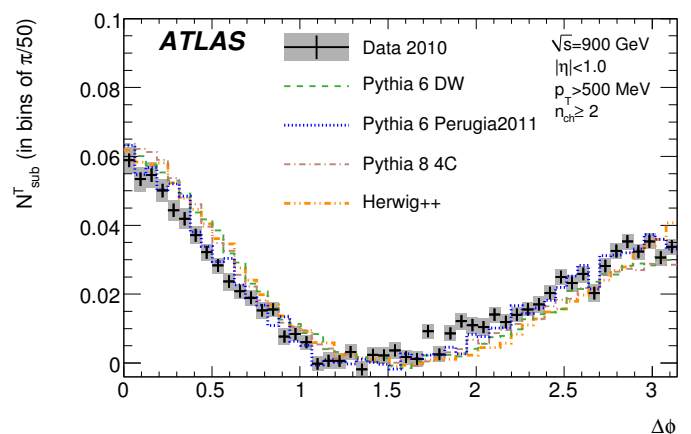

(a)

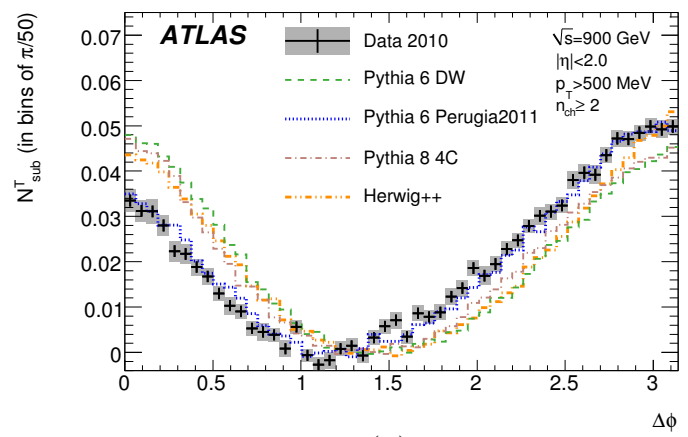

(c)

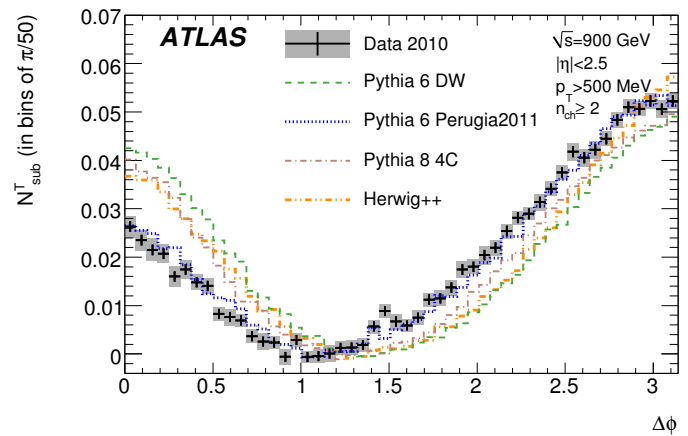

(e)

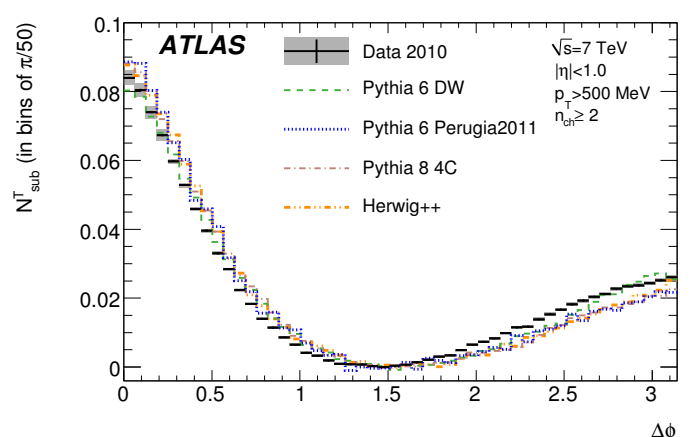

(b)

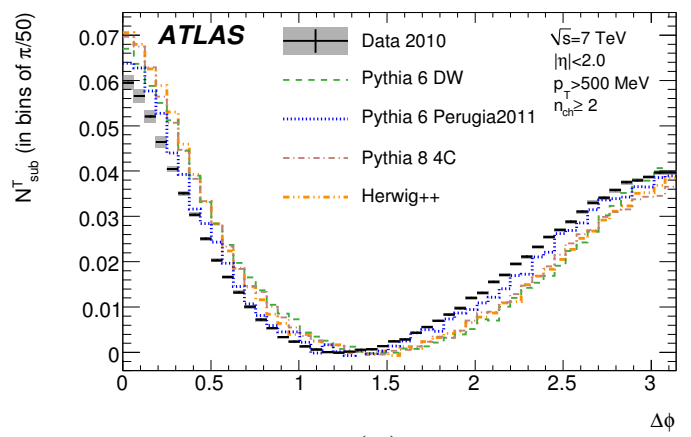

(d)

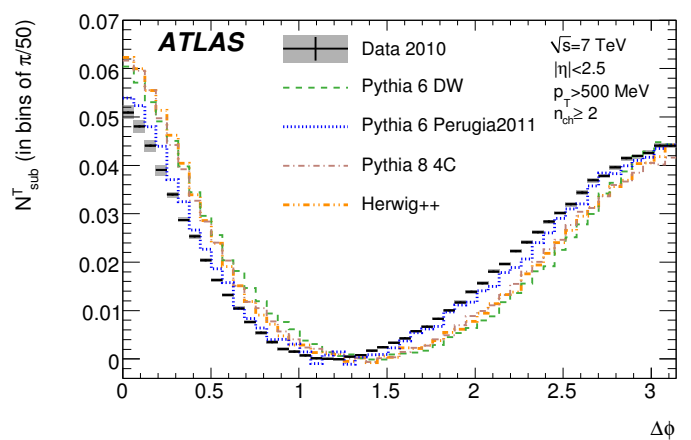

(f)

Figure 6. Subtracted and normalised $\Delta \phi$ distributions $\left(N_{\mathrm{sub}}^{T}\right)$ for data taken at $\sqrt{s}=900 \mathrm{GeV}$ (a, c, e) and $7 \mathrm{TeV}$ (b, d, f). Three different pseudorapidity regions are shown: $|\eta|<1.0,|\eta|<2.0$, $|\eta|<2.5$. The data (black symbols) are compared with the predictions of different MC tunes (see text for more details). The vertical bars on the data points denote the statistical uncertainty, while the shaded areas denote the total uncertainty. The leading particle in each event is omitted from the plot.

$7 \mathrm{TeV}$, and by somewhat more at $900 \mathrm{GeV}$; the shapes of the distributions are generally similar but some of the tunes show systematically different trends from the data. With the summed- $p_{\mathrm{T}}$ correlation, similar conclusions are found. It should be noted that present Monte Carlo generators have not been tuned using these correlations.

Both types of correlation are found to be significantly stronger at $7 \mathrm{TeV}$ than at $900 \mathrm{GeV}$, the relative increase being greater at larger intervals in pseudorapidity. This 


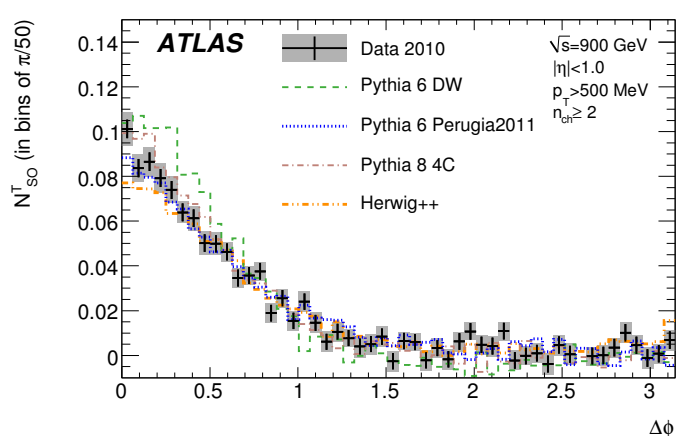

(a)

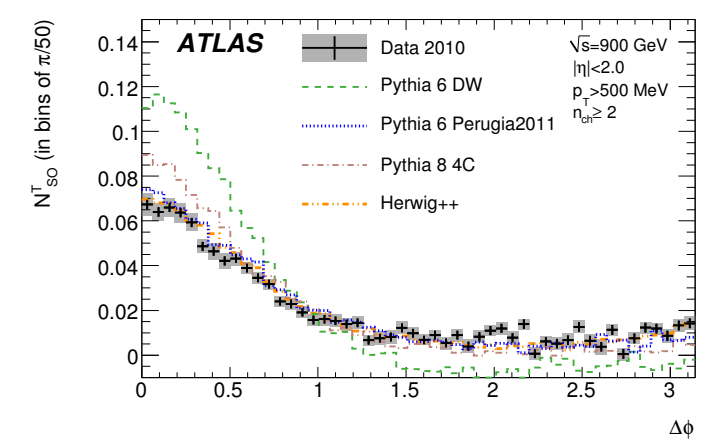

(c)

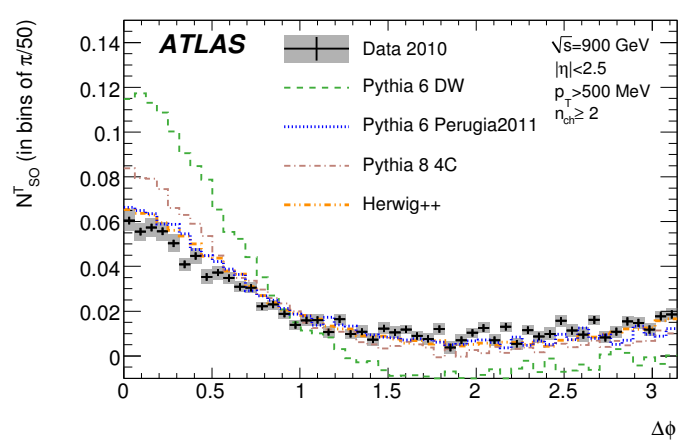

(e)

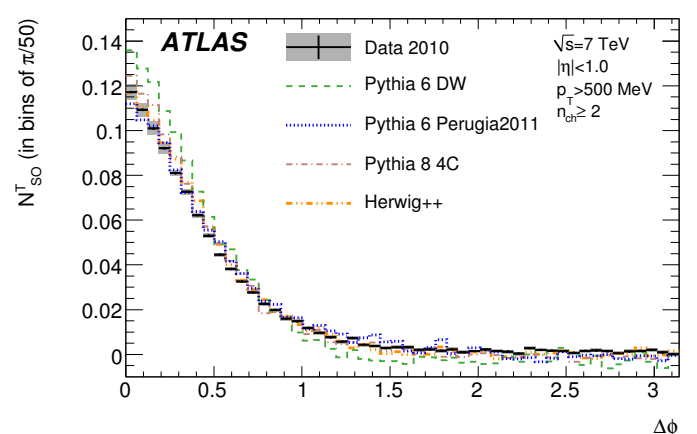

(b)

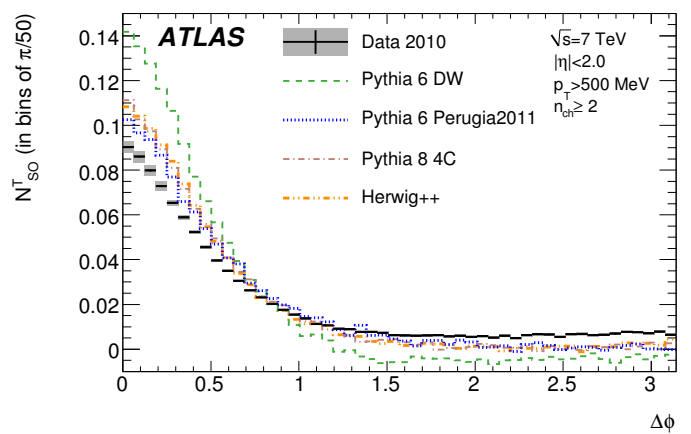

(d)

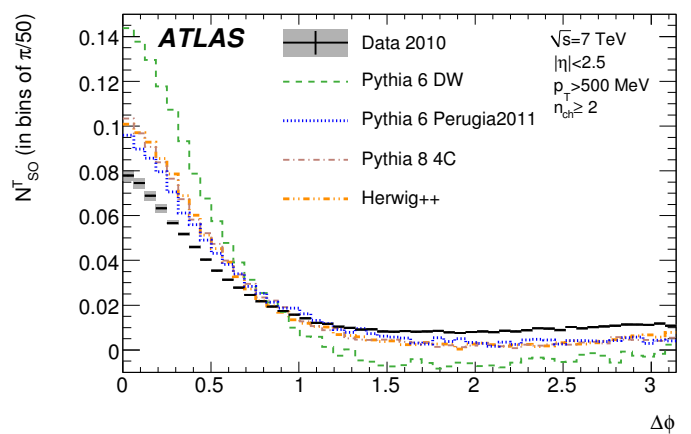

(f)

Figure 7. Normalised "same hemisphere minus opposite" distributions $\left(N_{\mathrm{SO}}^{T}\right)$ for data taken at $\sqrt{s}=900 \mathrm{GeV}$ (a, c, e) and $7 \mathrm{TeV}$ (b, d, f). Three different pseudorapidity regions are shown: $|\eta|<$ $1.0,|\eta|<2.0,|\eta|<2.5$. The data (black symbols) are compared with the predictions of different MC tunes (see text for more details). The vertical bars on the data points denote the statistical uncertainty, while the shaded areas denote the total uncertainty. The leading particle in each event is omitted from the plot.

trend is reproduced in most of the MC models. As expected, a strong tendency is seen for the multiplicity correlations to fall with increasing minimum transverse momentum of the charged particles. This illustrates well the transition between the soft, non-perturbative regime of parton string or cluster fragmentation and the jet-dominated regime of perturbative quantum chromodynamics.

Azimuthal charged-particle distributions relative to the leading charged particle have 
been measured by means of two variables, using the full pseudorapidity reach of the ATLAS tracking detectors. These variables minimise inter-jet effects in different ways, and give different sensitivities to the jet-like properties of the event distributions. In both cases, the MC models give fair to good descriptions of the data, provided that the selected pseudorapidity range is within one unit of zero. As the range in pseudorapidity is increased, however, disagreements with most of the models become more pronounced, in describing both the jet-influenced region near the leading charged particle and also the opposite-side region. This indicates the complexity of the effects that the models are seeking to describe when broad features of the events are being considered.

The event features studied here provide new means of discriminating between the different possible tunes of the Monte Carlo models. They will need to be taken into account, if the most accurate description of particle processes at the highest LHC energies is to be attained.

\section{Acknowledgments}

We thank CERN for the very successful operation of the LHC, as well as the support staff from our institutions without whom ATLAS could not be operated efficiently.

We acknowledge the support of ANPCyT, Argentina; YerPhI, Armenia; ARC, Australia; BMWF, Austria; ANAS, Azerbaijan; SSTC, Belarus; CNPq and FAPESP, Brazil; NSERC, NRC and CFI, Canada; CERN; CONICYT, Chile; CAS, MOST and NSFC, China; COLCIENCIAS, Colombia; MSMT CR, MPO CR and VSC CR, Czech Republic; DNRF, DNSRC and Lundbeck Foundation, Denmark; EPLANET and ERC, European Union; IN2P3-CNRS, CEA-DSM/IRFU, France; GNAS, Georgia; BMBF, DFG, HGF, MPG and AvH Foundation, Germany; GSRT, Greece; ISF, MINERVA, GIF, DIP and Benoziyo Center, Israel; INFN, Italy; MEXT and JSPS, Japan; CNRST, Morocco; FOM and NWO, Netherlands; RCN, Norway; MNiSW, Poland; GRICES and FCT, Portugal; MERYS (MECTS), Romania; MES of Russia and ROSATOM, Russian Federation; JINR; MSTD, Serbia; MSSR, Slovakia; ARRS and MVZT, Slovenia; DST/NRF, South Africa; MICINN, Spain; SRC and Wallenberg Foundation, Sweden; SER, SNSF and Cantons of Bern and Geneva, Switzerland; NSC, Taiwan; TAEK, Turkey; STFC, the Royal Society and Leverhulme Trust, United Kingdom; DOE and NSF, United States of America.

The crucial computing support from all WLCG partners is acknowledged gratefully, in particular from CERN and the ATLAS Tier-1 facilities at TRIUMF (Canada), NDGF (Denmark, Norway, Sweden), CC-IN2P3 (France), KIT/GridKA (Germany), INFN-CNAF (Italy), NL-T1 (Netherlands), PIC (Spain), ASGC (Taiwan), RAL (U.K.) and BNL (USA) and in the Tier-2 facilities worldwide.

\section{A Summary of Monte Carlo tunes}

Table 5 summarises the main features of the PYTHIA- and HERWIG-based Monte Carlo tunes employed in the analysis and referred to in the text. 


\begin{tabular}{|c|c|c|c|c|}
\hline Generator & Tune & $\begin{array}{c}\text { Shower } \\
\text { ordering }\end{array}$ & PDF & Additional information \\
\hline \hline \multirow{3}{*}{ PYTHIA 6.4 } & DW & virtuality & CTEQ 5L & Non-interleaved MPI model \\
\cline { 2 - 5 } & MC09 & $p_{\mathrm{T}}$ & MRSTLO* $^{*}$ & MPI interleaved with ISR \\
\cline { 2 - 5 } & Perugia 2011 & $p_{\mathrm{T}}$ & CTEQ 5L & MPI interleaved with ISR \\
\cline { 2 - 5 } PYTHIA 8.150 & AMB2B & $p_{\mathrm{T}}$ & CTEQ 6L1 & MPI interleaved with ISR \\
\hline \multirow{3}{*}{ HERWIG++ 2.5 } & $p_{\mathrm{T}}$ & CTEQ 6L1 & $\begin{array}{c}\text { MPI interleaved with ISR } \\
\text { and FSR; updated } \\
\text { model for diffraction }\end{array}$ \\
\cline { 2 - 6 } & MU900-2 & angle & $\begin{array}{c}\text { MRST } \\
2007 L L^{*}\end{array}$ & $\begin{array}{c}\text { MB tune for 900 GeV only; } \\
\text { no model for diffraction }\end{array}$ \\
\cline { 2 - 5 } & UE7-2 & angle & $\begin{array}{c}\text { MRST } \\
2007 \mathrm{LO}^{*}\end{array}$ & $\begin{array}{c}\text { UE tune for 7 TeV only; } \\
\text { no model for diffraction }\end{array}$ \\
\hline
\end{tabular}

Table 5. Main features of the different PYTHIA and HERWIG tunes used in the comparisons with the data, as used with CTEQ [37, 38] and MRST [39] parton distribution functions.

Open Access. This article is distributed under the terms of the Creative Commons Attribution License which permits any use, distribution and reproduction in any medium, provided the original author(s) and source are credited.

\section{References}

[1] W. Kittel and E.A. de Wolf, Soft Multihadron Dynamics, World Scientific, Singapore (2005).

[2] A. Buckley et al., General-purpose event generators for LHC physics, Phys. Rept. 504 (2011) 145 [arXiv: 1101.2599] [INSPIRE].

[3] T. Sjöstrand et al., High-energy physics event generation with PYTHIA 6.1, Comput. Phys. Commun. 135 (2001) 238 [hep-ph/0010017] [INSPIRE].

[4] T. Sjöstrand, S. Mrenna and P.Z. Skands, PYTHIA 6.4 physics and manual, JHEP 05 (2006) 026 [hep-ph/0603175] [INSPIRE].

[5] T. Sjöstrand, S. Mrenna and P.Z. Skands, A brief introduction to PYTHIA 8.1, Comput. Phys. Commun. 178 (2008) 852 [arXiv:0710.3820] [InSPIRE].

[6] C.M. Buttar et al., The underlying event, in Proceedings of Workshop HERA and the LHC, CERN, Geneva Switzerland (2004), DESY-PROC-2005-001 (2005) 183.

[7] A. Buckley, H. Hoeth, H. Lacker, H. Schulz and J.E. von Seggern, Systematic event generator tuning for the LHC, Eur. Phys. J. C 65 (2010) 331 [arXiv:0907.2973] [INSPIRE].

[8] ATLAS collaboration, ATLAS tunes of PYTHIA 6 and PYTHIA 8 for MC11, PHYS-PUB-2011-009 (2011).

[9] B. Andersson, G. Gustafson, G. Ingelman and T. Sjöstrand, Parton Fragmentation and String Dynamics, Phys. Rept. 97 (1983) 31 [InSPIRE].

[10] T. Sjöstrand, The Lund Monte Carlo for Jet Fragmentation and $e^{+} e^{-}$Physics: JETSET Version 6.2, Comput. Phys. Commun. 39 (1986) 347 [INSPIRE]. 
[11] A. Kupco, Cluster Hadronisation in Herwig 5.9, in Proceedings of Workshop Monte Carlo Generators for HERA Physics, Hamburg Germany (1998), A.T. Doyle et al. eds., DESY-PROC-1999-02 (1999), pg. 292.

[12] M. Bähr et al., Herwig++ 2.1 release note, Eur. Phys. J. C 58 (2008) 639 [arXiv: 0803.0883] [INSPIRE].

[13] S. Gieseke et al., Herwig++ 2.5 release note, arXiv:1102.1672 [INSPIRE].

[14] K. Wraight and P. Skands, Forward-Backward Correlations and Event Shapes as Probes of Minimum-Bias Event Properties, Eur. Phys. J. C 71 (2011) 1628 [arXiv:1101.5215] [INSPIRE].

[15] ATLAS collaboration, G. Aad et al., The ATLAS Experiment at the CERN Large Hadron Collider, 2008 JINST 3 S08003 [INSPIRE].

[16] UA5 collaboration, R. Ansorge et al., Charged particle correlations in p̄p collisions at c.m. energies of $200 \mathrm{GeV}, 546 \mathrm{GeV}$ and $900 \mathrm{GeV}$, Z. Phys. C 37 (1988) 191 [inSPIRE].

[17] ATLAS collaboration, G. Aad et al., Charged-particle multiplicities in pp interactions measured with the ATLAS detector at the LHC, New J. Phys. 13 (2011) 053033 [arXiv: 1012.5104] [INSPIRE].

[18] CMS collaboration, V. Khachatryan et al., Charged particle multiplicities in pp interactions at $\sqrt{s}=0.9$, 2.36 and $7 \mathrm{TeV}$, JHEP 01 (2011) 079 [arXiv:1011.5531] [INSPIRE].

[19] LHCB collaboration, R. Aaij et al., Measurement of charged particle multiplicities in pp collisions at $\sqrt{s}=7 \mathrm{TeV}$ in the forward region, Eur. Phys. J. C 72 (2012) 1947 [arXiv:1112.4592] [INSPIRE].

[20] ALICE collaboration, K. Aamodt et al., Charged-particle multiplicity measurement in proton-proton collisions at $\sqrt{s}=0.9$ and $2.36 \mathrm{TeV}$ with ALICE at LHC, Eur. Phys. J. C 68 (2010) 89 [arXiv: 1004.3034] [INSPIRE].

[21] ALICE collaboration, K. Aamodt et al., Charged-particle multiplicity measurement in proton-proton collisions at $\sqrt{s}=7 \mathrm{TeV}$ with ALICE at LHC, Eur. Phys. J. C 68 (2010) 345 [arXiv: 1004.3514] [INSPIRE].

[22] CMS collaboration, V. Khachatryan et al., Observation of Long-Range Near-Side Angular Correlations in Proton-Proton Collisions at the LHC, JHEP 09 (2010) 091 [arXiv: 1009.4122] [INSPIRE].

[23] ATLAS collaboration, G. Aad et al., Measurement of inclusive two-particle angular correlations in pp collisions with the ATLAS detector at the LHC, JHEP 05 (2012) 157 [arXiv:1203.3549] [INSPIRE].

[24] CDF collaboration, D. Acosta et al., The underlying event in hard interactions at the Tevatron $\bar{p} p$ collider, Phys. Rev. D 70 (2004) 072002 [hep-ex/0404004] [INSPIRE].

[25] CDF collaboration, T. Aaltonen et al., Studying the Underlying Event in Drell-Yan and High Transverse Momentum Jet Production at the Tevatron, Phys. Rev. D 82 (2010) 034001 [arXiv: 1003.3146] [INSPIRE].

[26] D0 collaboration, Study of $\phi$ and $\eta$ correlations in minimum bias events with the D0 detector at the Fermilab Tevatron Collider, D0 Note 6054-CONF (2010).

[27] ATLAS collaboration, G. Aad et al., Measurements of underlying-event properties using neutral and charged particles in pp collisions at $900 \mathrm{GeV}$ and $7 \mathrm{TeV}$ with the ATLAS detector at the LHC, Eur. Phys. J. C 71 (2011) 1636 [arXiv:1103.1816] [InSPIRE]. 
[28] ATLAS collaboration, ATLAS Monte Carlo tunes for MC09, PHYS-PUB-2010-002 (2010).

[29] ATLAS collaboration, G. Aad et al., The ATLAS Simulation Infrastructure, Eur. Phys. J. C 70 (2010) 823 [arXiv: 1005.4568] [InSPIRE].

[30] GEANT4 collaboration, S. Agostinelli et al., GEANT4: A Simulation toolkit, Nucl. Instrum. Meth. A 506 (2003) 250 [INSPIRE].

[31] R. Field, Early LHC Underlying Event Data - Findings and Surprises, arXiv:1010.3558 [INSPIRE].

[32] P.Z. Skands, Tuning Monte Carlo Generators: The Perugia Tunes, Phys. Rev. D 82 (2010) 074018 [arXiv: 1005.3457] [INSPIRE].

[33] R. Corke and T. Sjöstrand, Interleaved parton showers and tuning prospects, JHEP 03 (2011) 032 [arXiv:1011.1759] [INSPIRE].

[34] R. Engel, Photoproduction within the two component dual parton model. 1. Amplitudes and cross-sections, Z. Phys. C 66 (1995) 203 [INSPIRE].

[35] ATLAS collaboration, ATLAS tunes of PYTHIA 6 and PYTHIA 8 for MC11, PHYS-PUB-2011-009 (2011).

[36] M. Kendall and A. Stuart, The Advanced Theory of Statistics. Vol. 2, 3rd edition, Charles Griffin \& Co., London U.K. (1951).

[37] CTEQ collaboration, H. Lai et al., Global QCD analysis of parton structure of the nucleon: CTEQ5 parton distributions, Eur. Phys. J. C 12 (2000) 375 [hep-ph/9903282] [INSPIRE].

[38] J. Pumplin et al., New generation of parton distributions with uncertainties from global QCD analysis, JHEP 07 (2002) 012 [hep-ph/0201195] [INSPIRE].

[39] A. Sherstnev and R. Thorne, Parton Distributions for LO Generators, Eur. Phys. J. C 55 (2008) 553 [arXiv:0711.2473] [INSPIRE].

\section{The ATLAS collaboration}

G. Aad $^{48}$, B. Abbott ${ }^{110}$, J. Abdallah ${ }^{11}$, A.A. Abdelalim ${ }^{49}$, A. Abdesselam ${ }^{117}$, O. Abdinov ${ }^{10}$, B. Abi ${ }^{111}$, M. Abolins ${ }^{87}$, O.S. AbouZeid ${ }^{157}$, H. Abramowicz ${ }^{152}$, H. Abreu ${ }^{114}$, E. Acerbi ${ }^{88 a, 88 b}$, B.S. Acharya ${ }^{163 a, 163 b}$, L. Adamczyk ${ }^{37}$, D.L. Adams ${ }^{24}$, T.N. Addy ${ }^{56}$, J. Adelman ${ }^{174}$, M. Aderholz ${ }^{98}$, S. Adomeit ${ }^{97}$, P. Adragna ${ }^{74}$, T. Adye ${ }^{128}$, S. Aefsky ${ }^{22}$, J.A. Aguilar-Saavedra ${ }^{123 b, a}$, M. Aharrouche ${ }^{80}$, S.P. Ahlen ${ }^{21}$, F. Ahles ${ }^{48}$, A. Ahmad $^{147}$, M. $\operatorname{Ahsan}^{40}$, G. Aielli ${ }^{132 a, 132 b}$, T. Akdogan ${ }^{18 a}$, T.P.A. Åkesson ${ }^{78}$, G. Akimoto ${ }^{154}$, A.V. Akimov ${ }^{93}$, A. Akiyama ${ }^{66}$, M.S. Alam ${ }^{1}$, M.A. Alam ${ }^{75}$, J. Albert ${ }^{168}$, S. Albrand ${ }^{55}$, M. Aleksa ${ }^{29}$, I.N. Aleksandrov ${ }^{64}$, F. Alessandria ${ }^{88 a}$, C. Alexa ${ }^{25 a}$, G. Alexander ${ }^{152}$, G. Alexandre ${ }^{49}$, T. Alexopoulos ${ }^{9}$, M. Alhroob ${ }^{20}$, M. Aliev ${ }^{15}$, G. Alimonti ${ }^{88 a}$, J. Alison ${ }^{119}$, M. Aliyev ${ }^{10}$, B.M.M. Allbrooke ${ }^{17}$, P.P. Allport ${ }^{72}$, S.E. Allwood-Spiers ${ }^{53}$, J. Almond ${ }^{81}$, A. Aloisio ${ }^{101 a, 101 b}$, R. Alon ${ }^{170}$, A. Alonso ${ }^{78}$, B. Alvarez Gonzalez ${ }^{87}$, M.G. Alviggi ${ }^{101 a, 101 b}$, K. Amako ${ }^{65}$, P. Amaral ${ }^{29}$, C. Amelung ${ }^{22}$, V.V. Ammosov ${ }^{127}$, A. Amorim ${ }^{123 a, b}$, G. Amorós ${ }^{166}$, N. Amram ${ }^{152}$, C. Anastopoulos ${ }^{29}$, L.S. Ancu $^{16}$, N. Andari ${ }^{114}$, T. Andeen ${ }^{34}$, C.F. Anders ${ }^{20}$, G. Anders ${ }^{58 a}$, K.J. Anderson ${ }^{30}$, A. Andreazza ${ }^{88 a, 88 b}$, V. Andrei ${ }^{58 a}$, M-L. Andrieux ${ }^{55}$, X.S. Anduaga ${ }^{69}$, A. Angerami $^{34}$, 
F. Anghinolfi ${ }^{29}$, A. Anisenkov ${ }^{106}$, N. Anjos ${ }^{123 a}$, A. Annovi ${ }^{47}$, A. Antonaki ${ }^{8}$, M. Antonelli ${ }^{47}$, A. Antonov ${ }^{95}$, J. Antos ${ }^{143 b}$, F. Anulli ${ }^{131 a}$, S. Aoun ${ }^{82}$, L. Aperio Bella ${ }^{4}$, R. Apolle ${ }^{117, c}$, G. Arabidze ${ }^{87}$, I. Aracena ${ }^{142}$, Y. Arai ${ }^{65}$, A.T.H. Arce ${ }^{44}$, S. Arfaoui ${ }^{147}$, J-F. Arguin ${ }^{14}$, E. Arik ${ }^{18 a}$,*, M. Arik ${ }^{18 a}$, A.J. Armbruster ${ }^{86}$, O. Arnaez ${ }^{80}$, C. Arnault ${ }^{114}$, A. Artamonov ${ }^{94}$, G. Artoni ${ }^{131 a, 131 b}$, D. Arutinov ${ }^{20}$, S. Asai ${ }^{154}$, R. Asfandiyarov ${ }^{171}$, S. Ask ${ }^{27}$, B. Åsman ${ }^{145 a, 145 b}$, L. Asquith ${ }^{5}$, K. Assamagan ${ }^{24}$, A. Astbury ${ }^{168}$,

A. Astvatsatourov ${ }^{52}$, B. Aubert ${ }^{4}$, E. Auge ${ }^{114}$, K. Augsten ${ }^{126}$, M. Aurousseau ${ }^{144 a}$, G. Avolio ${ }^{162}$, R. Avramidou ${ }^{9}$, D. Axen ${ }^{167}$, C. Ay ${ }^{54}$, G. Azuelos ${ }^{92, d}$, Y. Azuma ${ }^{154}$, M.A. Baak ${ }^{29}$, G. Baccaglioni ${ }^{88 a}$, C. Bacci ${ }^{133 a, 133 b}$, A.M. Bach ${ }^{14}$, H. Bachacou ${ }^{135}$, K. Bachas ${ }^{29}$, M. Backes ${ }^{49}$, M. Backhaus ${ }^{20}$, E. Badescu ${ }^{25 a}$, P. Bagnaia ${ }^{131 a, 131 b}$, S. Bahinipati ${ }^{2}$, Y. Bai ${ }^{32 a}$, D.C. Bailey ${ }^{157}$, T. Bain ${ }^{157}$, J.T. Baines ${ }^{128}$, O.K. Baker ${ }^{174}$, M.D. Baker ${ }^{24}$, S. Baker ${ }^{76}$, E. Banas ${ }^{38}$, P. Banerjee ${ }^{92}$, Sw. Banerjee ${ }^{171}$, D. Banfi ${ }^{29}$, A. Bangert ${ }^{149}$, V. Bansal ${ }^{168}$, H.S. Bansil ${ }^{17}$, L. Barak ${ }^{170}$, S.P. Baranov ${ }^{93}$, A. Barashkou ${ }^{64}$, A. Barbaro Galtieri ${ }^{14}$, T. Barber ${ }^{48}$, E.L. Barberio ${ }^{85}$, D. Barberis ${ }^{50 a, 50 b}$, M. Barbero ${ }^{20}$, D.Y. Bardin ${ }^{64}$, T. Barillari ${ }^{98}$, M. Barisonzi ${ }^{173}$, T. Barklow ${ }^{142}$, N. Barlow ${ }^{27}$, B.M. Barnett ${ }^{128}$, R.M. Barnett ${ }^{14}$, A. Baroncelli ${ }^{133 a}$, G. Barone ${ }^{49}$, A.J. Barr ${ }^{117}$, F. Barreiro ${ }^{79}$, J. Barreiro Guimarães da Costa $^{57}$, P. Barrillon ${ }^{114}$, R. Bartoldus ${ }^{142}$, A.E. Barton ${ }^{70}$, V. Bartsch ${ }^{148}$, R.L. Bates ${ }^{53}$, L. Batkova ${ }^{143 a}$, J.R. Batley ${ }^{27}$, A. Battaglia ${ }^{16}$, M. Battistin ${ }^{29}$, F. Bauer ${ }^{135}$, H.S. Bawa ${ }^{142, e}$, S. Beale ${ }^{97}$, T. Beau ${ }^{77}$, P.H. Beauchemin ${ }^{160}$, R. Beccherle ${ }^{50 a}$, P. Bechtle ${ }^{20}$, H.P. Beck ${ }^{16}$, S. Becker ${ }^{97}$, M. Beckingham ${ }^{137}$, K.H. Becks ${ }^{173}$, A.J. Beddall ${ }^{18 c}$, A. Beddall ${ }^{18 c}$, S. Bedikian ${ }^{174}$, V.A. Bednyakov ${ }^{64}$, C.P. Bee ${ }^{82}$, M. Begel $^{24}$, S. Behar Harpaz ${ }^{151}$, P.K. Behera ${ }^{62}$, M. Beimforde ${ }^{98}$, C. Belanger-Champagne ${ }^{84}$, P.J. Bell ${ }^{49}$, W.H. Bell ${ }^{49}$, G. Bella ${ }^{152}$, L. Bellagamba ${ }^{19 a}$, F. Bellina ${ }^{29}$, M. Bellomo ${ }^{29}$, A. Belloni ${ }^{57}$, O. Beloborodova ${ }^{106, f}$, K. Belotskiy ${ }^{95}$, O. Beltramello ${ }^{29}$, S. Ben Ami ${ }^{151}$, O. Benary ${ }^{152}$, D. Benchekroun ${ }^{134 a}$, C. Benchouk ${ }^{82}$, M. Bendel ${ }^{80}$, N. Benekos ${ }^{164}$, Y. Benhammou ${ }^{152}$, E. Benhar Noccioli ${ }^{49}$, J.A. Benitez Garcia ${ }^{158 b}$, D.P. Benjamin ${ }^{44}$, M. Benoit ${ }^{114}$, J.R. Bensinger ${ }^{22}$, K. Benslama ${ }^{129}$, S. Bentvelsen ${ }^{104}$, D. Berge ${ }^{29}$,

E. Bergeaas Kuutmann ${ }^{41}$, N. Berger ${ }^{4}$, F. Berghaus ${ }^{168}$, E. Berglund ${ }^{104}$, J. Beringer ${ }^{14}$, P. Bernat ${ }^{76}$, R. Bernhard ${ }^{48}$, C. Bernius ${ }^{24}$, T. Berry ${ }^{75}$, C. Bertella ${ }^{82}$, A. Bertin ${ }^{19 a, 19 b}$,

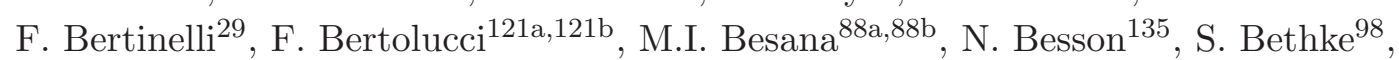
W. Bhimji ${ }^{45}$, R.M. Bianchi ${ }^{29}$, M. Bianco ${ }^{71 a, 71 b}$, O. Biebel ${ }^{97}$, S.P. Bieniek ${ }^{76}$, K. Bierwagen ${ }^{54}$, J. Biesiada ${ }^{14}$, M. Biglietti ${ }^{133 a}$, H. Bilokon ${ }^{47}$, M. Bindi19a,19b ${ }^{19}$ S. Binet ${ }^{114}$, A. Bingul ${ }^{18 c}$, C. Bini1 ${ }^{131 a, 131 b}$, C. Biscarat ${ }^{176}$, U. Bitenc ${ }^{48}$, K.M. Black ${ }^{21}$, R.E. Blair ${ }^{5}$, J.-B. Blanchard ${ }^{135}$, G. Blanchot ${ }^{29}$, T. Blazek ${ }^{143 a}$, C. Blocker ${ }^{22}$, J. Blocki ${ }^{38}$, A. Blondel ${ }^{49}$, W. Blum ${ }^{80}$, U. Blumenschein ${ }^{54}$, G.J. Bobbink ${ }^{104}$, V.B. Bobrovnikov ${ }^{106}$, S.S. Bocchetta ${ }^{78}$, A. Bocci ${ }^{44}$, C.R. Boddy ${ }^{117}$, M. Boehler ${ }^{41}$, J. Boek ${ }^{173}$, N. Boelaert ${ }^{35}$, J.A. Bogaerts ${ }^{29}$,

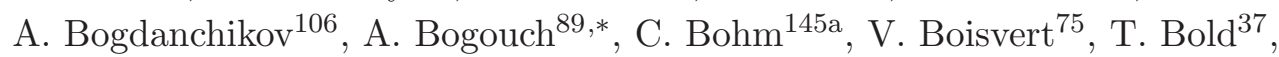
V. Boldea ${ }^{25 a}$, N.M. Bolnet ${ }^{135}$, M. Bona ${ }^{74}$, V.G. Bondarenko ${ }^{95}$, M. Bondioli ${ }^{162}$, M. Boonekamp ${ }^{135}$, C.N. Booth ${ }^{138}$, S. Bordoni ${ }^{77}$, C. Borer ${ }^{16}$, A. Borisov ${ }^{127}$, G. Borissov ${ }^{70}$, I. Borjanovic ${ }^{12 \mathrm{a}}$, M. Borri ${ }^{81}$, S. Borroni ${ }^{86}$, V. Bortolotto ${ }^{133 a, 133 b}$, K. Bos ${ }^{104}$, D. Boscherini ${ }^{19 a}$, M. Bosman ${ }^{11}$, H. Boterenbrood ${ }^{104}$, D. Botterill ${ }^{128}$, J. Bouchami ${ }^{92}$, J. Boudreau ${ }^{122}$, E.V. Bouhova-Thacker ${ }^{70}$, D. Boumediene ${ }^{33}$, C. Bourdarios ${ }^{114}$, N. Bousson ${ }^{82}$, A. Boveia ${ }^{30}$, J. Boyd ${ }^{29}$, I.R. Boyko ${ }^{64}$, N.I. Bozhko ${ }^{127}$, 
I. Bozovic-Jelisavcic ${ }^{12 b}$, J. Bracinik ${ }^{17}$, A. Braem ${ }^{29}$, P. Branchini1 ${ }^{133 a}$, G.W. Brandenburg ${ }^{57}$, A. Brandt ${ }^{7}$, G. Brandt ${ }^{117}$, O. Brandt ${ }^{54}$, U. Bratzler ${ }^{155}$, B. Brau ${ }^{83}$, J.E. Brau ${ }^{113}$, H.M. Braun ${ }^{173}$, B. Brelier ${ }^{157}$, J. Bremer ${ }^{29}$, R. Brenner ${ }^{165}$, S. Bressler ${ }^{170}$, D. Britton ${ }^{53}$, F.M. Brochu ${ }^{27}$, I. Brock ${ }^{20}$, R. Brock ${ }^{87}$, T.J. Brodbeck ${ }^{70}$, E. Brodet ${ }^{152}$, F. Broggi ${ }^{88 a}$, C. Bromberg ${ }^{87}$, J. Bronner ${ }^{98}$, G. Brooijmans ${ }^{34}$, W.K. Brooks ${ }^{31 b}$, G. Brown ${ }^{81}$, H. Brown ${ }^{7}$, P.A. Bruckman de Renstrom ${ }^{38}$, D. Bruncko ${ }^{143 b}$, R. Bruneliere ${ }^{48}$, S. Brunet ${ }^{60}$, A. Bruni ${ }^{19 a}$, G. Bruni ${ }^{19 a}$, M. Bruschi ${ }^{19 a}$, T. Buanes ${ }^{13}$, Q. Buat ${ }^{55}$, F. Bucci ${ }^{49}$, J. Buchanan ${ }^{117}$, N.J. Buchanan ${ }^{2}$, P. Buchholz ${ }^{140}$, R.M. Buckingham ${ }^{117}$, A.G. Buckley ${ }^{45}$, S.I. Buda ${ }^{25 a}$, I.A. Budagov ${ }^{64}$, B. Budick ${ }^{107}$, V. Büscher ${ }^{80}$, L. Bugge ${ }^{116}$, O. Bulekov ${ }^{95}$, M. Bunse ${ }^{42}$, T. Buran ${ }^{116}$, H. Burckhart ${ }^{29}$, S. Burdin ${ }^{72}$, T. Burgess ${ }^{13}$, S. Burke ${ }^{128}$, E. Busato ${ }^{33}$, P. Bussey ${ }^{53}$, C.P. Buszello ${ }^{165}$, F. Butin ${ }^{29}$, B. Butler ${ }^{142}$, J.M. Butler ${ }^{21}$, C.M. Buttar ${ }^{53}$, J.M. Butterworth ${ }^{76}$, W. Buttinger ${ }^{27}$, S. Cabrera Urbán ${ }^{166}$, D. Caforio ${ }^{19 a, 19 b}$, O. Cakir $^{3 a}$, P. Calafiura ${ }^{14}$, G. Calderini ${ }^{77}$, P. Calfayan ${ }^{97}$, R. Calkins ${ }^{105}$, L.P. Caloba ${ }^{23 a}$, R. Caloi ${ }^{131 a, 131 b}$, D. Calvet ${ }^{33}$, S. Calvet $^{33}$, R. Camacho Toro ${ }^{33}$, P. Camarri ${ }^{132 a, 132 b}$, M. Cambiaghi ${ }^{118 a, 118 b}$, D. Cameron ${ }^{116}$, L.M. Caminada ${ }^{14}$, S. Campana ${ }^{29}$, M. Campanelli ${ }^{76}$, V. Canale ${ }^{101 a, 101 b}$, F. Canelli ${ }^{30, g}$, A. Canepa ${ }^{158 a}$, J. Cantero ${ }^{79}$, L. Capasso ${ }^{101 a, 101 b}$, M.D.M. Capeans Garrido ${ }^{29}$, I. Caprini' ${ }^{25 a}$, M. Caprini ${ }^{25 a}$, D. Capriotti ${ }^{98}$, M. Capua ${ }^{36 a, 36 b}$, R. Caputo $^{80}$, C. Caramarcu $^{24}$, R. Cardarelli ${ }^{132 a}$, T. Carli ${ }^{29}$, G. Carlino ${ }^{101 a}$, L. Carminati ${ }^{88 a, 88 b}$, B. Caron ${ }^{84}$, S. Caron $^{103}$, G.D. Carrillo Montoya ${ }^{171}$, A.A. Carter ${ }^{74}$, J.R. Carter $^{27}$, J. Carvalho ${ }^{123 a, h}$, D. Casadei ${ }^{107}$, M.P. Casado ${ }^{11}$, M. Cascella121a,121b , C. Caso ${ }^{50 a, 50 b, *}$, A.M. Castaneda Hernandez ${ }^{171}$, E. Castaneda-Miranda ${ }^{171}$, V. Castillo Gimenez ${ }^{166}$, N.F. Castro ${ }^{123 a}$, G. Cataldi ${ }^{71 a}$, F. Cataneo ${ }^{29}$, A. Catinaccio ${ }^{29}$, J.R. Catmore ${ }^{29}$, A. Cattai ${ }^{29}$, G. Cattani ${ }^{132 a}, 132 b$, S. Caughron ${ }^{87}$, D. Cauz ${ }^{163 a, 163 c}$, P. Cavalleri ${ }^{77}$, D. Cavalli ${ }^{88 a}$, M. Cavalli-Sforza ${ }^{11}$, V. Cavasinni ${ }^{121 a, 121 b}$, F. Ceradini ${ }^{133 a, 133 b}$, A.S. Cerqueira ${ }^{23 b}$, A. Cerri ${ }^{29}$, L. Cerrito ${ }^{74}$, F. Cerutti $^{47}$, S.A. Cetin ${ }^{18 b}$, F. Cevenini101a,101b ${ }^{1}$ A. Chafaq ${ }^{134 a}$, D. Chakraborty ${ }^{105}$, K. Chan ${ }^{2}$, B. Chapleau ${ }^{84}$, J.D. Chapman ${ }^{27}$, J.W. Chapman ${ }^{86}$, E. Chareyre ${ }^{77}$, D.G. Charlton ${ }^{17}$, V. Chavda ${ }^{81}$, C.A. Chavez Barajas ${ }^{29}$, S. Cheatham ${ }^{84}$, S. Chekanov ${ }^{5}$, S.V. Chekulaev ${ }^{158 a}$, G.A. Chelkov ${ }^{64}$, M.A. Chelstowska ${ }^{103}$, C. Chen ${ }^{63}$, H. Chen ${ }^{24}$, S. Chen ${ }^{32 c}$, T. Chen ${ }^{32 c}$, X. Chen ${ }^{171}$, S. Cheng ${ }^{32 a}$, A. Cheplakov ${ }^{64}$, V.F. Chepurnov ${ }^{64}$, R. Cherkaoui El Moursli ${ }^{134 \mathrm{e}}$, V. Chernyatin ${ }^{24}$, E. Cheu ${ }^{6}$, S.L. Cheung ${ }^{157}$, L. Chevalier ${ }^{135}$, G. Chiefari ${ }^{101 a, 101 b}$, L. Chikovani ${ }^{51 a}$, J.T. Childers ${ }^{29}$, A. Chilingarov ${ }^{70}$, G. Chiodini ${ }^{71 a}$, A.S. Chisholm ${ }^{17}$, M.V. Chizhov ${ }^{64}$, G. Choudalakis $^{30}$, S. Chouridou ${ }^{136}$, I.A. Christidi ${ }^{76}$, A. Christov $^{48}$, D. Chromek-Burckhart ${ }^{29}$, M.L. Chu ${ }^{150}$, J. Chudoba ${ }^{124}$, G. Ciapetti1 ${ }^{131 a, 131 b}$, K. Ciba ${ }^{37}$, A.K. Ciftci ${ }^{3 a}$, R. Ciftci $^{3 a}$, D. Cinca ${ }^{33}$, V. Cindro ${ }^{73}$, M.D. Ciobotaru ${ }^{162}$, C. Ciocca ${ }^{19 a}$, A. Ciocio $^{14}$, M. Cirilli ${ }^{86}$, M. Citterio ${ }^{88 a}$,

M. Ciubancan ${ }^{25 a}$, A. Clark ${ }^{49}$, P.J. Clark ${ }^{45}$, W. Cleland ${ }^{122}$, J.C. Clemens ${ }^{82}$, B. Clement ${ }^{55}$, C. Clement ${ }^{145 a, 145 b}$, R.W. Clifft ${ }^{128}$, Y. Coadou ${ }^{82}$, M. Cobal ${ }^{163 a, 163 c}$, A. Coccaro ${ }^{171}$, J. Cochran ${ }^{63}$, P. Coe ${ }^{117}$, J.G. Cogan ${ }^{142}$, J. Coggeshall ${ }^{164}$, E. Cogneras ${ }^{176}$, J. Colas ${ }^{4}$, A.P. Colijn ${ }^{104}$, N.J. Collins ${ }^{17}$, C. Collins-Tooth ${ }^{53}$, J. Collot ${ }^{55}$, G. Colon ${ }^{83}$, P. Conde Muiño $^{123 a}$, E. Coniavitis ${ }^{117}$, M.C. Conidi ${ }^{11}$, M. Consonni ${ }^{103}$, V. Consorti ${ }^{48}$, S. Constantinescu ${ }^{25 a}$, C. Conta ${ }^{118 a, 118 b}$, F. Conventi101a, $i$, J. Cook ${ }^{29}$, M. Cooke ${ }^{14}$, B.D. Cooper ${ }^{76}$, A.M. Cooper-Sarkar ${ }^{117}$, K. Copic ${ }^{14}$, T. Cornelissen ${ }^{173}$, M. Corradi ${ }^{19 a}$, 


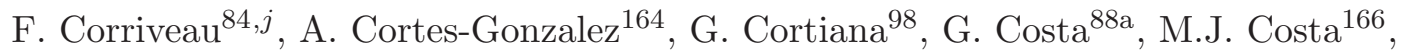

D. Costanzo ${ }^{138}$, T. Costin $^{30}$, D. Côtéé 29 , R. Coura Torres ${ }^{23 a}$, L. Courneyea ${ }^{168}$,

G. Cowan $^{75}$, C. Cowden ${ }^{27}$, B.E. Cox ${ }^{81}$, K. Cranmer ${ }^{107}$, F. Crescioli ${ }^{121 a, 121 b}$,

M. Cristinziani ${ }^{20}$, G. Crosetti ${ }^{36 a, 36 b}$, R. Crupi ${ }^{71 a, 71 b}$, S. Crépé-Renaudin ${ }^{55}$,

C.-M. Cuciuc ${ }^{25 a}$, C. Cuenca Almenar ${ }^{174}$, T. Cuhadar Donszelmann ${ }^{138}$, M. Curatolo ${ }^{47}$,

C.J. Curtis ${ }^{17}$, C. Cuthbert ${ }^{149}$, P. Cwetanski ${ }^{60}$, H. Czirr ${ }^{140}$, P. Czodrowski ${ }^{43}$,

Z. Czyczula ${ }^{174}$, S. D'Auria ${ }^{53}$, M. D'Onofrio ${ }^{72}$, A. D'Orazio ${ }^{131 a, 131 b}$, P.V.M. Da Silva ${ }^{23 a}$,

C. Da Via ${ }^{81}$, W. Dabrowski ${ }^{37}$, T. Dai ${ }^{86}$, C. Dallapiccola ${ }^{83}$, M. Dam ${ }^{35}$, M. Dameri ${ }^{50 a, 50 b}$,

D.S. Damiani ${ }^{136}$, H.O. Danielsson ${ }^{29}$, D. Dannheim ${ }^{98}$, V. Dao ${ }^{49}$, G. Darbo ${ }^{50 a}$,

G.L. Darlea ${ }^{25 b}$, W. Davey ${ }^{20}$, T. Davidek ${ }^{125}$, N. Davidson ${ }^{85}$, R. Davidson ${ }^{70}$, E. Davies ${ }^{117, c}$,

M. Davies ${ }^{92}$, A.R. Davison ${ }^{76}$, Y. Davygora ${ }^{58 a}$, E. Dawe ${ }^{141}$, I. Dawson ${ }^{138}$, J.W. Dawson ${ }^{5, *}$,

R.K. Daya-Ishmukhametova ${ }^{22}, K_{\text {. De }}^{7}$, R. de Asmundis ${ }^{101 a}$, S. De Castro ${ }^{19 a, 19 b}$,

P.E. De Castro Faria Salgado ${ }^{24}$, S. De Cecco ${ }^{77}$, J. de Graat ${ }^{97}$, N. De Groot ${ }^{103}$,

P. de Jong ${ }^{104}$, C. De La Taille ${ }^{114}$, H. De la Torre ${ }^{79}$, B. De Lotto ${ }^{163 a, 163 c}$, L. de Mora ${ }^{70}$,

L. De Nooij ${ }^{104}$, D. De Pedis ${ }^{131 a}$, A. De Salvo ${ }^{131 a}$, U. De Sanctis ${ }^{163 a, 163 c}$, A. De Santo ${ }^{148}$,

J.B. De Vivie De Regie ${ }^{114}$, S. Dean ${ }^{76}$, W.J. Dearnaley ${ }^{70}$, R. Debbe ${ }^{24}$, C. Debenedetti ${ }^{45}$,

D.V. Dedovich ${ }^{64}$, J. Degenhardt ${ }^{119}$, M. Dehchar ${ }^{117}$, C. Del Papa ${ }^{163 a, 163 c}$, J. Del Peso ${ }^{79}$,

T. Del Prete ${ }^{121 \mathrm{a}, 121 \mathrm{~b}}$, T. Delemontex ${ }^{55}$, M. Deliyergiyev ${ }^{73}$, A. Dell'Acqua ${ }^{29}$,

L. Dell'Asta ${ }^{21}$, M. Della Pietra ${ }^{101 a, i}$, D. della Volpe ${ }^{101 a, 101 b}$, M. Delmastro ${ }^{4}$,

N. Delruelle ${ }^{29}$, P.A. Delsart ${ }^{55}$, C. Deluca ${ }^{147}$, S. Demers ${ }^{174}$, M. Demichev ${ }^{64}$,

B. Demirkoz ${ }^{11, k}$, J. Deng ${ }^{162}$, S.P. Denisov ${ }^{127}$, D. Derendarz ${ }^{38}$, J.E. Derkaoui134d,

F. Derue ${ }^{77}$, P. Dervan ${ }^{72}$, K. Desch ${ }^{20}$, E. Devetak ${ }^{147}$, P.O. Deviveiros ${ }^{104}$, A. Dewhurst ${ }^{128}$,

B. DeWilde ${ }^{147}$, S. Dhaliwal ${ }^{157}$, R. Dhullipudi ${ }^{24, l}$, A. Di Ciaccio ${ }^{132 a, 132 b}$, L. Di Ciaccio ${ }^{4}$,

A. Di Girolamo ${ }^{29}$, B. Di Girolamo ${ }^{29}$, S. Di Luise ${ }^{133 a, 133 b}$, A. Di Mattia ${ }^{171}$, B. Di Micco ${ }^{29}$,

R. Di Nardo ${ }^{47}$, A. Di Simone132a,132b, R. Di Sipio ${ }^{19 a, 19 b}$, M.A. Diaz ${ }^{31 a}$, F. Diblen ${ }^{18 c}$,

E.B. Diehl ${ }^{86}$, J. Dietrich ${ }^{41}$, T.A. Dietzsch ${ }^{58 a}$, S. Diglio ${ }^{85}$, K. Dindar Yagci ${ }^{39}$,

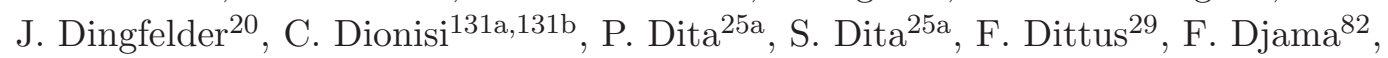

T. Djobava ${ }^{51 b}$, M.A.B. do Vale ${ }^{23 c}$, A. Do Valle Wemans ${ }^{123 a}$, T.K.O. Doan ${ }^{4}$, M. Dobbs ${ }^{84}$,

R. Dobinson ${ }^{29, *}$, D. Dobos ${ }^{29}$, E. Dobson ${ }^{29, m}$, J. Dodd $^{34}$, C. Doglioni ${ }^{49}$, T. Doherty ${ }^{53}$,

Y. Doi ${ }^{65, *}$, J. Dolejsi ${ }^{125}$, I. Dolenc ${ }^{73}$, Z. Dolezal ${ }^{125}$, B.A. Dolgoshein ${ }^{95, *}$, T. Dohmae ${ }^{154}$,

M. Donadelli2 ${ }^{23 d}$, M. Donega ${ }^{119}$, J. Donini ${ }^{33}$, J. Dopke ${ }^{29}$, A. Doria ${ }^{101 a}$, A. Dos Anjos ${ }^{171}$,

M. Dosil ${ }^{11}$, A. Dotti ${ }^{121 a, 121 b}$, M.T. Dova ${ }^{69}$, J.D. Dowell ${ }^{17}$, A.D. Doxiadis ${ }^{104}$,

A.T. Doyle ${ }^{53}$, Z. Drasal ${ }^{125}$, J. Drees ${ }^{173}$, N. Dressnandt ${ }^{119}$, H. Drevermann ${ }^{29}$,

C. Driouichi ${ }^{35}$, M. Dris ${ }^{9}$, J. Dubbert ${ }^{98}$, S. Dube ${ }^{14}$, E. Duchovni ${ }^{170}$, G. Duckeck ${ }^{97}$,

A. Dudarev ${ }^{29}$, F. Dudziak ${ }^{63}$, M. Dührssen ${ }^{29}$, I.P. Duerdoth ${ }^{81}$, L. Duflot ${ }^{114}$,

M-A. Dufour ${ }^{84}$, M. Dunford ${ }^{29}$, H. Duran Yildiz ${ }^{3 a}$, R. Duxfield ${ }^{138}$, M. Dwuznik ${ }^{37}$,

F. Dydak ${ }^{29}$, M. Düren ${ }^{52}$, W.L. Ebenstein ${ }^{44}$, J. Ebke ${ }^{97}$, S. Eckweiler ${ }^{80}$, K. Edmonds ${ }^{80}$,

C.A. Edwards ${ }^{75}$, N.C. Edwards ${ }^{53}$, W. Ehrenfeld ${ }^{41}$, T. Ehrich $^{98}$, T. Eifert ${ }^{142}$, G. Eigen ${ }^{13}$,

K. Einsweiler ${ }^{14}$, E. Eisenhandler ${ }^{74}$, T. Ekelof ${ }^{165}$, M. El Kacimi ${ }^{134 c}$, M. Ellert ${ }^{165}$, S. Elles ${ }^{4}$,

F. Ellinghaus ${ }^{80}$, K. Ellis ${ }^{74}$, N. Ellis ${ }^{29}$, J. Elmsheuser ${ }^{97}$, M. Elsing ${ }^{29}$, D. Emeliyanov ${ }^{128}$,

R. Engelmann ${ }^{147}$, A. Engl ${ }^{97}$, B. $\operatorname{Epp}^{61}$, A. Eppig ${ }^{86}$, J. Erdmann ${ }^{54}$, A. Ereditato ${ }^{16}$,

D. Eriksson ${ }^{145 a}$, J. Ernst ${ }^{1}$, M. Ernst ${ }^{24}$, J. Ernwein ${ }^{135}$, D. Errede ${ }^{164}$, S. Errede ${ }^{164}$,

E. Ertel $^{80}$, M. Escalier ${ }^{114}$, C. Escobar ${ }^{122}$, X. Espinal Curull ${ }^{11}$, B. Esposito ${ }^{47}$, 
F. Etienne ${ }^{82}$, A.I. Etienvre ${ }^{135}$, E. Etzion ${ }^{152}$, D. Evangelakou ${ }^{54}$, H. Evans ${ }^{60}$,

L. Fabbri ${ }^{19 a, 19 b}$, C. Fabre ${ }^{29}$, R.M. Fakhrutdinov ${ }^{127}$, S. Falciano ${ }^{131 a}$, Y. Fang ${ }^{171}$,

M. Fanti ${ }^{88 a, 88 b}$, A. Farbin ${ }^{7}$, A. Farilla ${ }^{133 a}$, J. Farley ${ }^{147}$, T. Farooque ${ }^{157}$,

S.M. Farrington ${ }^{117}$, P. Farthouat ${ }^{29}$, P. Fassnacht ${ }^{29}$, D. Fassouliotis ${ }^{8}$, B. Fatholahzadeh ${ }^{157}$,

A. Favareto ${ }^{88 a, 88 b}$, L. Fayard ${ }^{114}$, S. Fazio ${ }^{36 a, 36 b}$, R. Febbraro ${ }^{33}$, P. Federic ${ }^{143 a}$,

O.L. Fedin ${ }^{120}$, W. Fedorko ${ }^{87}$, M. Fehling-Kaschek ${ }^{48}$, L. Feligioni ${ }^{82}$, D. Fellmann ${ }^{5}$,

C. Feng ${ }^{32 d}$, E.J. Feng ${ }^{30}$, A.B. Fenyuk ${ }^{127}$, J. Ferencei ${ }^{143 b}$, J. Ferland ${ }^{92}$, W. Fernando ${ }^{108}$,

S. Ferrag ${ }^{53}$, J. Ferrando ${ }^{53}$, V. Ferrara ${ }^{41}$, A. Ferrari ${ }^{165}$, P. Ferrari ${ }^{104}$, R. Ferrari ${ }^{118 a}$,

D.E. Ferreira de Lima ${ }^{53}$, A. Ferrer ${ }^{166}$, M.L. Ferrer ${ }^{47}$, D. Ferrere ${ }^{49}$, C. Ferretti ${ }^{86}$,

A. Ferretto Parodi ${ }^{50 a, 50 b}$, M. Fiascaris ${ }^{30}$, F. Fiedler ${ }^{80}$, A. Filipčič ${ }^{73}$, A. Filippas ${ }^{9}$,

F. Filthaut ${ }^{103}$, M. Fincke-Keeler ${ }^{168}$, M.C.N. Fiolhais ${ }^{123 a, h}$, L. Fiorini ${ }^{166}$, A. Firan ${ }^{39}$,

G. Fischer ${ }^{41}$, P. Fischer ${ }^{20}$, M.J. Fisher ${ }^{108}$, M. Flechl ${ }^{48}$, I. Fleck ${ }^{140}$, J. Fleckner ${ }^{80}$,

P. Fleischmann ${ }^{172}$, S. Fleischmann ${ }^{173}$, T. Flick ${ }^{173}$, A. Floderus ${ }^{78}$, L.R. Flores Castillo ${ }^{171}$,

M.J. Flowerdew ${ }^{98}$, M. Fokitis ${ }^{9}$, T. Fonseca Martin ${ }^{16}$, D.A. Forbush ${ }^{137}$, A. Formica ${ }^{135}$,

A. Forti ${ }^{81}$, D. Fortin ${ }^{158 a}$, J.M. Foster ${ }^{81}$, D. Fournier ${ }^{114}$, A. Foussat ${ }^{29}$, A.J. Fowler ${ }^{44}$,

K. Fowler ${ }^{136}$, H. Fox ${ }^{70}$, P. Francavilla ${ }^{11}$, S. Franchino ${ }^{118 a, 118 b}$, D. Francis ${ }^{29}$, T. Frank ${ }^{170}$,

M. Franklin ${ }^{57}$, S. Franz ${ }^{29}$, M. Fraternali118a,118b , S. Fratina ${ }^{119}$, S.T. French ${ }^{27}$,

F. Friedrich ${ }^{43}$, R. Froeschl ${ }^{29}$, D. Froidevaux ${ }^{29}$, J.A. Frost ${ }^{27}$, C. Fukunaga ${ }^{155}$,

E. Fullana Torregrosa ${ }^{29}$, J. Fuster ${ }^{166}$, C. Gabaldon ${ }^{29}$, O. Gabizon ${ }^{170}$, T. Gadfort ${ }^{24}$,

S. Gadomski ${ }^{49}$, G. Gagliardi ${ }^{50 a, 50 b}$, P. Gagnon ${ }^{60}$, C. Galea ${ }^{97}$, E.J. Gallas ${ }^{117}$, V. Gallo ${ }^{16}$,

B.J. Gallop ${ }^{128}$, P. Gallus ${ }^{124}$, K.K. Gan ${ }^{108}$, Y.S. Gao ${ }^{142, e}$, V.A. Gapienko ${ }^{127}$,

A. Gaponenko ${ }^{14}$, F. Garberson ${ }^{174}$, M. Garcia-Sciveres ${ }^{14}$, C. García ${ }^{166}$, J.E. García

Navarro $^{166}$, R.W. Gardner ${ }^{30}$, N. Garelli ${ }^{29}$, H. Garitaonandia ${ }^{104}$, V. Garonne ${ }^{29}$,

J. Garvey ${ }^{17}$, C. Gatti ${ }^{47}$, G. Gaudio ${ }^{118 a}$, B. Gaur ${ }^{140}$, L. Gauthier ${ }^{135}$, I.L. Gavrilenko ${ }^{93}$,

C. Gay ${ }^{167}$, G. Gaycken ${ }^{20}$, J-C. Gayde ${ }^{29}$, E.N. Gazis ${ }^{9}$, P. Ge ${ }^{32 d}$, C.N.P. Gee ${ }^{128}$,

D.A.A. Geerts ${ }^{104}$, Ch. Geich-Gimbel ${ }^{20}$, K. Gellerstedt ${ }^{145 a, 145 b}$, C. Gemme ${ }^{50 a}$,

A. Gemmell ${ }^{53}$, M.H. Genest ${ }^{55}$, S. Gentile ${ }^{131 a, 131 b}$, M. George ${ }^{54}$, S. George ${ }^{75}$,

P. Gerlach ${ }^{173}$, A. Gershon ${ }^{152}$, C. Geweniger ${ }^{58 a}$, H. Ghazlane ${ }^{134 b}$, N. Ghodbane ${ }^{33}$,

B. Giacobbe ${ }^{19 a}$, S. Giagu ${ }^{131 a, 131 b}$, V. Giakoumopoulou ${ }^{8}$, V. Giangiobbe ${ }^{11}$, F. Gianotti ${ }^{29}$,

B. Gibbard $^{24}$, A. Gibson ${ }^{157}$, S.M. Gibson ${ }^{29}$, L.M. Gilbert ${ }^{117}$, V. Gilewsky ${ }^{90}$,

D. Gillberg ${ }^{28}$, A.R. Gillman ${ }^{128}$, D.M. Gingrich ${ }^{2, d}$, J. Ginzburg ${ }^{152}$, N. Giokaris ${ }^{8}$,

M.P. Giordani ${ }^{163 c}$, R. Giordano ${ }^{101 a, 101 b}$, F.M. Giorgi ${ }^{15}$, P. Giovannini ${ }^{98}$, P.F. Giraud ${ }^{135}$,

D. Giugni ${ }^{88 a}$, M. Giunta ${ }^{92}$, P. Giusti ${ }^{19 a}$, B.K. Gjelsten ${ }^{116}$, L.K. Gladilin ${ }^{96}$, C. Glasman ${ }^{79}$,

J. Glatzer ${ }^{48}$, A. Glazov ${ }^{41}$, K.W. Glitza ${ }^{173}$, G.L. Glonti ${ }^{64}$, J.R. Goddard ${ }^{74}$, J. Godfrey ${ }^{141}$,

J. Godlewski ${ }^{29}$, M. Goebel ${ }^{41}$, T. Göpfert ${ }^{43}$, C. Goeringer ${ }^{80}$, C. Gössling ${ }^{42}$, T. Göttfert ${ }^{98}$,

S. Goldfarb ${ }^{86}$, T. Golling ${ }^{174}$, A. Gomes ${ }^{123 a, b}$, L.S. Gomez Fajardo ${ }^{41}$, R. Gonçalo ${ }^{75}$,

J. Goncalves Pinto Firmino Da Costa ${ }^{41}$, L. Gonella ${ }^{20}$, A. Gonidec ${ }^{29}$, S. Gonzalez ${ }^{171}$,

S. González de la Hoz ${ }^{166}$, G. Gonzalez Parra ${ }^{11}$, M.L. Gonzalez Silva ${ }^{26}$,

S. Gonzalez-Sevilla ${ }^{49}$, J.J. Goodson ${ }^{147}$, L. Goossens ${ }^{29}$, P.A. Gorbounov ${ }^{94}$, H.A. Gordon ${ }^{24}$,

I. Gorelov ${ }^{102}$, G. Gorfine ${ }^{173}$, B. Gorini ${ }^{29}$, E. Gorini ${ }^{71 a, 71 b}$, A. Gorišek ${ }^{73}$, E. Gornicki ${ }^{38}$,

S.A. Gorokhov ${ }^{127}$, V.N. Goryachev ${ }^{127}$, B. Gosdzik ${ }^{41}$, M. Gosselink ${ }^{104}$, M.I. Gostkin ${ }^{64}$,

I. Gough Eschrich ${ }^{162}$, M. Gouighri ${ }^{134 a}$, D. Goujdami ${ }^{134 c}$, M.P. Goulette ${ }^{49}$,

A.G. Goussiou ${ }^{137}$, C. Goy ${ }^{4}$, S. Gozpinar ${ }^{22}$, I. Grabowska-Bold ${ }^{37}$, P. Grafström ${ }^{29}$, 
K-J. Grahn ${ }^{41}$, F. Grancagnolo ${ }^{71 a}$, S. Grancagnolo ${ }^{15}$, V. Grassi ${ }^{147}$, V. Gratchev ${ }^{120}$, N. Grau ${ }^{34}$, H.M. Gray ${ }^{29}$, J.A. Gray ${ }^{147}$, E. Graziani ${ }^{133 a}$, O.G. Grebenyuk ${ }^{120}$,

T. Greenshaw ${ }^{72}$, Z.D. Greenwood ${ }^{24, l}$, K. Gregersen ${ }^{35}$, I.M. Gregor ${ }^{41}$, P. Grenier ${ }^{142}$,

J. Griffiths ${ }^{137}$, N. Grigalashvili ${ }^{64}$, A.A. Grillo ${ }^{136}$, S. Grinstein ${ }^{11}$, Y.V. Grishkevich ${ }^{96}$, J.-F. Grivaz ${ }^{114}$, M. Groh ${ }^{98}$, E. Gross ${ }^{170}$, J. Grosse-Knetter ${ }^{54}$, J. Groth-Jensen ${ }^{170}$, K. Grybel ${ }^{140}$, V.J. Guarino ${ }^{5}$, D. Guest ${ }^{174}$, C. Guicheney ${ }^{33}$, A. Guida ${ }^{71 a, 71 b}$, S. Guindon ${ }^{54}$, H. Guler ${ }^{84, n}$, J. Gunther ${ }^{124}$, B. Guo ${ }^{157}$, J. Guo ${ }^{34}$, A. Gupta $^{30}$, Y. Gusakov ${ }^{64}$, V.N. Gushchin ${ }^{127}$, P. Gutierrez ${ }^{110}$, N. Guttman ${ }^{152}$, O. Gutzwiller ${ }^{171}$, C. Guyot ${ }^{135}$, C. Gwenlan ${ }^{117}$, C.B. Gwilliam ${ }^{72}$, A. Haas ${ }^{142}$, S. $\operatorname{Haas}^{29}$, C. Haber ${ }^{14}$, H.K. Hadavand ${ }^{39}$, D.R. Hadley ${ }^{17}$, P. Haefner ${ }^{98}$, F. Hahn ${ }^{29}$, S. Haider ${ }^{29}$, Z. Hajduk ${ }^{38}$, H. Hakobyan ${ }^{175}$, D. Hall ${ }^{117}$, J. Haller ${ }^{54}$, K. Hamacher ${ }^{173}$, P. Hamal ${ }^{112}$, M. Hamer ${ }^{54}$, A. Hamilton ${ }^{144 b, o}$,

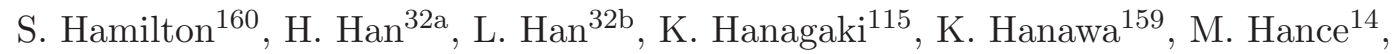
C. Handel ${ }^{80}$, P. Hanke ${ }^{58 a}$, J.R. Hansen ${ }^{35}$, J.B. Hansen ${ }^{35}$, J.D. Hansen ${ }^{35}$, P.H. Hansen ${ }^{35}$, P. Hansson ${ }^{142}$, K. Hara ${ }^{159}$, G.A. Hare ${ }^{136}$, T. Harenberg ${ }^{173}$, S. Harkusha ${ }^{89}$, D. Harper ${ }^{86}$, R.D. Harrington ${ }^{45}$, O.M. Harris ${ }^{137}$, K. Harrison ${ }^{17}$, J. Hartert ${ }^{48}$, F. Hartjes ${ }^{104}$,

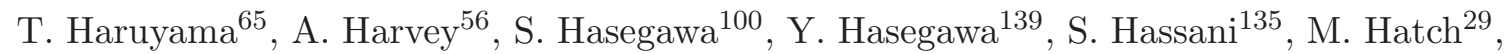
D. Hauff ${ }^{98}$, S. Haug ${ }^{16}$, M. Hauschild ${ }^{29}$, R. Hauser ${ }^{87}$, M. Havranek ${ }^{20}$, B.M. Hawes ${ }^{117}$, C.M. Hawkes ${ }^{17}$, R.J. Hawkings ${ }^{29}$, A.D. Hawkins ${ }^{78}$, D. Hawkins ${ }^{162}$, T. Hayakawa ${ }^{66}$, T. Hayashi ${ }^{159}$, D. Hayden ${ }^{75}$, H.S. Hayward ${ }^{72}$, S.J. Haywood ${ }^{128}$, E. Hazen ${ }^{21}$, M. He ${ }^{32 d}$, S.J. Head ${ }^{17}$, V. Hedberg ${ }^{78}$, L. Heelan ${ }^{7}$, S. Heim ${ }^{87}$, B. Heinemann ${ }^{14}$, S. Heisterkamp ${ }^{35}$, L. Helary ${ }^{4}$, C. Heller ${ }^{97}$, M. Heller ${ }^{29}$, S. Hellman ${ }^{145 a, 145 b}$, D. Hellmich ${ }^{20}$, C. Helsens ${ }^{11}$, R.C.W. Henderson ${ }^{70}$, M. Henke ${ }^{58 a}$, A. Henrichs ${ }^{54}$, A.M. Henriques Correia ${ }^{29}$,

S. Henrot-Versille ${ }^{114}$, F. Henry-Couannier ${ }^{82}$, C. Hensel ${ }^{54}$, T. Henß ${ }^{173}$, C.M. Hernandez ${ }^{7}$, Y. Hernández Jiménez ${ }^{166}$, R. Herrberg ${ }^{15}$, A.D. Hershenhorn ${ }^{151}$, G. Herten ${ }^{48}$, R. Hertenberger ${ }^{97}$, L. Hervas ${ }^{29}$, G.G. Hesketh ${ }^{76}$, N.P. Hessey ${ }^{104}$, E. Higón-Rodriguez ${ }^{166}$, D. Hill ${ }^{5, *}$, J.C. Hill ${ }^{27}$, N. Hill ${ }^{5}$, K.H. Hiller ${ }^{41}$, S. Hillert ${ }^{20}$, S.J. Hillier ${ }^{17}$, I. Hinchliffe ${ }^{14}$,

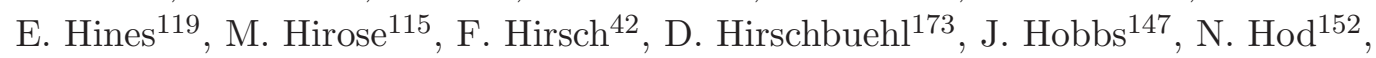
M.C. Hodgkinson ${ }^{138}$, P. Hodgson ${ }^{138}$, A. Hoecker ${ }^{29}$, M.R. Hoeferkamp ${ }^{102}$, J. Hoffman ${ }^{39}$, D. Hoffmann ${ }^{82}$, M. Hohlfeld ${ }^{80}$, M. Holder ${ }^{140}$, S.O. Holmgren ${ }^{145 a}$, T. Holy ${ }^{126}$, J.L. Holzbauer ${ }^{87}$, Y. Homma ${ }^{66}$, T.M. Hong ${ }^{119}$, L. Hooft van Huysduynen ${ }^{107}$, T. Horazdovsky ${ }^{126}$, C. Horn ${ }^{142}$, S. Horner ${ }^{48}$, J-Y. Hostachy ${ }^{55}$, S. Hou ${ }^{150}$, M.A. Houlden ${ }^{72}$, A. Hoummada ${ }^{134 a}$, J. Howarth ${ }^{81}$, D.F. Howell ${ }^{117}$, I. Hristova ${ }^{15}$, J. Hrivnac ${ }^{114}$, I. Hruska ${ }^{124}$, T. Hryn'ova ${ }^{4}$, P.J. Hsu ${ }^{80}$, S.-C. Hsu ${ }^{14}$, G.S. Huang ${ }^{110}$, Z. Hubacek ${ }^{126}$, F. Hubaut ${ }^{82}$, F. Huegging ${ }^{20}$, A. Huettmann ${ }^{41}$, T.B. Huffman ${ }^{117}$, E.W. Hughes ${ }^{34}$, G. Hughes ${ }^{70}$, R.E. Hughes-Jones ${ }^{81}$, M. Huhtinen ${ }^{29}$, P. Hurst ${ }^{57}$, M. Hurwitz ${ }^{14}$, U. Husemann ${ }^{41}$, N. Huseynov ${ }^{64, p}$, J. Huston ${ }^{87}$, J. Huth ${ }^{57}$, G. Iacobucci ${ }^{49}$, G. Iakovidis ${ }^{9}$, M. Ibbotson ${ }^{81}$, I. Ibragimov ${ }^{140}$, R. Ichimiya ${ }^{66}$, L. Iconomidou-Fayard ${ }^{114}$, J. Idarraga ${ }^{114}$, P. Iengo ${ }^{101 a}$, O. Igonkina ${ }^{104}$, Y. Ikegami ${ }^{65}$, M. Ikeno ${ }^{65}$, Y. Ilchenko ${ }^{39}$, D. Iliadis ${ }^{153}$, N. Ilic ${ }^{157}$, M. Imori ${ }^{154}$, T. Ince ${ }^{20}$, J. Inigo-Golfin ${ }^{29}$, P. Ioannou ${ }^{8}$, M. Iodice $^{133 a}$, V. Ippolito ${ }^{131 \mathrm{a}, 131 \mathrm{~b}}$, A. Irles Quiles ${ }^{166}, \mathrm{C}$. Isaksson ${ }^{165}$, A. Ishikawa ${ }^{66}, \mathrm{M}$. Ishino ${ }^{67}$, R. Ishmukhametov ${ }^{39}$, C. Issever ${ }^{117}$, S. Istin ${ }^{18 a}$, A.V. Ivashin ${ }^{127}$, W. Iwanski ${ }^{38}$, H. Iwasaki ${ }^{65}$, J.M. Izen ${ }^{40}$, V. Izzo ${ }^{101 a}$, B. Jackson ${ }^{119}$, J.N. Jackson ${ }^{72}$, P. Jackson ${ }^{142}$, M.R. Jaekel ${ }^{29}$, V. Jain ${ }^{60}$, K. Jakobs ${ }^{48}$, S. Jakobsen ${ }^{35}$, J. Jakubek ${ }^{126}$, D.K. Jana ${ }^{110}$, 
E. Jankowski ${ }^{157}$, E. Jansen ${ }^{76}$, H. Jansen ${ }^{29}$, A. Jantsch ${ }^{98}$, M. Janus ${ }^{20}$, G. Jarlskog ${ }^{78}$, L. Jeanty ${ }^{57}$, K. Jelen ${ }^{37}$, I. Jen-La Plante ${ }^{30}$, P. Jenni ${ }^{29}$, A. Jeremie ${ }^{4}$, P. Jež ${ }^{35}$, S. Jézéquel ${ }^{4}$, M.K. Jha ${ }^{19 a}$, H. Ji ${ }^{171}$, W. Ji ${ }^{80}$, J. Jia ${ }^{147}$, Y. Jiang ${ }^{32 b}$, M. Jimenez Belenguer ${ }^{41}$, G. Jin ${ }^{32 b}$, S. Jin ${ }^{32 a}$, O. Jinnouchi ${ }^{156}$, M.D. Joergensen ${ }^{35}$, D. Joffe ${ }^{39}$, L.G. Johansen ${ }^{13}$, M. Johansen ${ }^{145 a, 145 b}$, K.E. Johansson ${ }^{145 a}$, P. Johansson ${ }^{138}$, S. Johnert ${ }^{41}$, K.A. Johns ${ }^{6}$, K. Jon-And ${ }^{145 a, 145 b}$, G. Jones ${ }^{117}$, R.W.L. Jones ${ }^{70}$, T.W. Jones ${ }^{76}$, T.J. Jones ${ }^{72}$, O. Jonsson ${ }^{29}$, C. Joram ${ }^{29}$, P.M. Jorge ${ }^{123 a}$, J. Joseph ${ }^{14}$, J. Jovicevic ${ }^{146}$, T. Jovin ${ }^{12 b}$, X. Ju${ }^{171}$, C.A. Jung ${ }^{42}$, R.M. Jungst ${ }^{29}$, V. Juranek ${ }^{124}$, P. Jussel ${ }^{61}$, A. Juste Rozas ${ }^{11}$, V.V. Kabachenko ${ }^{127}$, S. Kabana ${ }^{16}$, M. Kaci ${ }^{166}$, A. Kaczmarska ${ }^{38}$, P. Kadlecik ${ }^{35}$, M. Kado ${ }^{114}$, H. Kagan ${ }^{108}$, M. Kagan ${ }^{57}$, S. Kaiser ${ }^{98}$, E. Kajomovitz ${ }^{151}$, S. Kalinin ${ }^{173}$, L.V. Kalinovskaya ${ }^{64}$, S. Kama ${ }^{39}$, N. Kanaya ${ }^{154}$, M. Kaneda ${ }^{29}$, S. Kaneti ${ }^{27}$, T. Kanno ${ }^{156}$, V.A. Kantserov ${ }^{95}$, J. Kanzaki ${ }^{65}$, B. Kaplan ${ }^{174}$, A. Kapliy ${ }^{30}$, J. Kaplon ${ }^{29}$, D. Kar ${ }^{43}$, M. Karagounis ${ }^{20}$, M. Karagoz ${ }^{117}$, M. Karnevskiy ${ }^{41}$, K. Karr ${ }^{5}$, V. Kartvelishvili ${ }^{70}$, A.N. Karyukhin ${ }^{127}$, L. Kashif ${ }^{171}$, G. Kasieczka ${ }^{58 b}$, R.D. Kass ${ }^{108}$, A. Kastanas ${ }^{13}$, M. Kataoka ${ }^{4}$, Y. Kataoka ${ }^{154}$, E. Katsoufis ${ }^{9}$, J. Katzy ${ }^{41}$, V. Kaushik ${ }^{6}$, K. Kawagoe ${ }^{66}$, T. Kawamoto ${ }^{154}$, G. Kawamura ${ }^{80}$, M.S. Kayl ${ }^{104}$, V.A. Kazanin ${ }^{106}$, M.Y. Kazarinov ${ }^{64}$, R. Keeler ${ }^{168}$, R. Kehoe $^{39}$, M. Keil ${ }^{54}$, G.D. Kekelidze ${ }^{64}$, J. Kennedy ${ }^{97}$, C.J. Kenney ${ }^{142}$, M. Kenyon ${ }^{53}$, O. Kepka ${ }^{124}$, N. Kerschen ${ }^{29}$, B.P. Kerševan ${ }^{73}$, S. Kersten ${ }^{173}$, K. Kessoku ${ }^{154}$, J. Keung ${ }^{157}$, F. Khalil-zada ${ }^{10}$, H. Khandanyan ${ }^{164}$, A. Khanov ${ }^{111}$, D. Kharchenko ${ }^{64}$, A. Khodinov ${ }^{95}$, A.G. Kholodenko ${ }^{127}$, A. Khomich ${ }^{58 a}$, T.J. Khoo ${ }^{27}$, G. Khoriauli ${ }^{20}$, A. Khoroshilov ${ }^{173}$, N. Khovanskiy ${ }^{64}$, V. Khovanskiy ${ }^{94}$, E. Khramov ${ }^{64}$, J. Khubua ${ }^{51 b}$, H. Kim ${ }^{145 a, 145 b}$, M.S. Kim² ${ }^{2}$ S.H. Kim ${ }^{159}$, N. Kimura ${ }^{169}$, O. Kind ${ }^{15}$, B.T. King ${ }^{72}$, M. King ${ }^{66}$, R.S.B. King ${ }^{117}$, J. Kirk ${ }^{128}$, L.E. Kirsch ${ }^{22}$, A.E. Kiryunin ${ }^{98}$, T. Kishimoto ${ }^{66}$, D. Kisielewska ${ }^{37}$, T. Kittelmann ${ }^{122}$, A.M. Kiver ${ }^{127}$, E. Kladiva ${ }^{143 b}$, J. Klaiber-Lodewigs ${ }^{42}$, M. Klein ${ }^{72}$, U. Klein ${ }^{72}$, K. Kleinknecht ${ }^{80}$, M. Klemetti ${ }^{84}$, A. Klier ${ }^{170}$, P. Klimek ${ }^{145 a, 145 b}$, A. Klimentov ${ }^{24}$, R. Klingenberg ${ }^{42}$, J.A. Klinger ${ }^{81}$, E.B. Klinkby ${ }^{35}$, T. Klioutchnikova ${ }^{29}$, P.F. Klok ${ }^{103}$, S. Klous ${ }^{104}$, E.-E. Kluge ${ }^{58 a}$, T. Kluge ${ }^{72}$, P. Kluit ${ }^{104}$, S. Kluth ${ }^{98}$,

N.S. Knecht ${ }^{157}$, E. Kneringer ${ }^{61}$, J. Knobloch ${ }^{29}$, E.B.F.G. Knoops ${ }^{82}$, A. Knue ${ }^{54}$, B.R. Ko ${ }^{44}$, T. Kobayashi ${ }^{154}$, M. Kobel ${ }^{43}$, M. Kocian ${ }^{142}$, P. Kodys ${ }^{125}$, K. Köneke ${ }^{29}$, A.C. König ${ }^{103}$, S. Koenig ${ }^{80}$, L. Köpke ${ }^{80}$, F. Koetsveld ${ }^{103}$, P. Koevesarki ${ }^{20}$, T. Koffas ${ }^{28}$, E. Koffeman ${ }^{104}$, L.A. Kogan ${ }^{117}$, F. Kohn ${ }^{54}$, Z. Kohout ${ }^{126}$, T. Kohriki ${ }^{65}$, T. Koi ${ }^{142}$, T. Kokott ${ }^{20}$, G.M. Kolachev ${ }^{106}$, H. Kolanoski ${ }^{15}$, V. Kolesnikov ${ }^{64}$, I. Koletsou ${ }^{88 a}$, J. Koll ${ }^{87}$, M. Kollefrath ${ }^{48}$, S.D. Kolya ${ }^{81}$, A.A. Komar ${ }^{93}$, Y. Komori ${ }^{154}$, T. Kondo ${ }^{65}$, T. Kono ${ }^{41, q}$, A.I. Kononov ${ }^{48}$, R. Konoplich ${ }^{107, r}$, N. Konstantinidis ${ }^{76}$, A. Kootz ${ }^{173}$, S. Koperny ${ }^{37}$, K. Korcyl ${ }^{38}$, K. Kordas ${ }^{153}$, V. Koreshev ${ }^{127}$, A. Korn ${ }^{117}$, A. Korol ${ }^{106}$, I. Korolkov ${ }^{11}$, E.V. Korolkova ${ }^{138}$, V.A. Korotkov ${ }^{127}$, O. Kortner ${ }^{98}$, S. Kortner ${ }^{98}$, V.V. Kostyukhin ${ }^{20}$, M.J. Kotamäki ${ }^{29}$, S. Kotov ${ }^{98}$, V.M. Kotov ${ }^{64}$, A. Kotwal ${ }^{44}$, C. Kourkoumelis ${ }^{8}$, V. Kouskoura ${ }^{153}$, A. Koutsman ${ }^{158 a}$, R. Kowalewski ${ }^{168}$, T.Z. Kowalski ${ }^{37}$, W. Kozanecki ${ }^{135}$, A.S. Kozhin ${ }^{127}$, V. Kral ${ }^{126}$, V.A. Kramarenko ${ }^{96}$, G. Kramberger ${ }^{73}$, M.W. Krasny ${ }^{77}$, A. Krasznahorkay ${ }^{107}$, J. Kraus ${ }^{87}$, J.K. Kraus ${ }^{20}$, A. Kreisel ${ }^{152}$, F. Krejci ${ }^{126}$, J. Kretzschmar ${ }^{72}$, N. Krieger ${ }^{54}$, P. Krieger ${ }^{157}$, K. Kroeninger ${ }^{54}$, H. Kroha ${ }^{98}$, J. Kroll ${ }^{119}$, J. Kroseberg ${ }^{20}$, J. Krstic ${ }^{12 a}$, U. Kruchonak ${ }^{64}$, H. Krüger ${ }^{20}$, T. Kruker ${ }^{16}$, N. Krumnack ${ }^{63}$, Z.V. Krumshteyn ${ }^{64}$, A. Kruth ${ }^{20}$, T. Kubota ${ }^{85}$, S. Kuday ${ }^{3 a}$, S. Kuehn ${ }^{48}$, A. Kugel ${ }^{58 c}$, 
T. Kuhl ${ }^{41}$, D. Kuhn ${ }^{61}$, V. Kukhtin ${ }^{64}$, Y. Kulchitsky ${ }^{89}$, S. Kuleshov ${ }^{31 b}$, C. Kummer ${ }^{97}$, M. Kuna ${ }^{77}$, N. Kundu ${ }^{117}$, J. Kunkle ${ }^{119}$, A. Kupco ${ }^{124}$, H. Kurashige ${ }^{66}$, M. Kurata ${ }^{159}$, Y.A. Kurochkin ${ }^{89}$, V. Kus ${ }^{124}$, E.S. Kuwertz ${ }^{146}$, M. Kuze ${ }^{156}$, J. Kvita ${ }^{141}$, R. Kwee ${ }^{15}$,

A. La Rosa ${ }^{49}$, L. La Rotonda ${ }^{36 a, 36 b}$, L. Labarga ${ }^{79}$, J. Labbe $^{4}$, S. Lablak ${ }^{134 a}$,

C. Lacasta ${ }^{166}$, F. Lacava ${ }^{131 a, 131 b}$, H. Lacker ${ }^{15}$, D. Lacour ${ }^{77}$, V.R. Lacuesta ${ }^{166}$,

E. Ladygin ${ }^{64}$, R. Lafaye ${ }^{4}$, B. Laforge ${ }^{77}$, T. Lagouri ${ }^{79}$, S. Lai ${ }^{48}$, E. Laisne $^{55}$,

M. Lamanna ${ }^{29}$, C.L. Lampen ${ }^{6}$, W. Lampl ${ }^{6}$, E. Lancon ${ }^{135}$, U. Landgraf ${ }^{48}$,

M.P.J. Landon ${ }^{74}$, J.L. Lane ${ }^{81}$, C. Lange ${ }^{41}$, A.J. Lankford ${ }^{162}$, F. Lanni ${ }^{24}$, K. Lantzsch ${ }^{173}$,

S. Laplace ${ }^{77}$, C. Lapoire $^{20}$, J.F. Laporte ${ }^{135}$, T. Lari ${ }^{88 a}$, A.V. Larionov ${ }^{127}$, A. Larner ${ }^{117}$,

C. Lasseur ${ }^{29}$, M. Lassnig ${ }^{29}$, P. Laurelli ${ }^{47}$, V. Lavorini ${ }^{36 a, 36 b}$, W. Lavrijsen ${ }^{14}$, P. Laycock ${ }^{72}$,

A.B. Lazarev ${ }^{64}$, O. Le Dortz ${ }^{77}$, E. Le Guirriec ${ }^{82}$, C. Le Maner ${ }^{157}$, E. Le Menedeu ${ }^{9}$,

C. Lebel ${ }^{92}$, T. LeCompte ${ }^{5}$, F. Ledroit-Guillon ${ }^{55}$, H. Lee ${ }^{104}$, J.S.H. Lee ${ }^{115}$, S.C. Lee ${ }^{150}$,

L. Lee ${ }^{174}$, M. Lefebvre ${ }^{168}$, M. Legendre ${ }^{135}$, A. Leger ${ }^{49}$, B.C. LeGeyt ${ }^{119}$, F. Legger ${ }^{97}$,

C. Leggett ${ }^{14}$, M. Lehmacher ${ }^{20}$, G. Lehmann Miotto ${ }^{29}$, X. Lei ${ }^{6}$, M.A.L. Leite ${ }^{23 d}$,

R. Leitner ${ }^{125}$, D. Lellouch ${ }^{170}$, M. Leltchouk ${ }^{34}$, B. Lemmer ${ }^{54}$, V. Lendermann ${ }^{58 a}$,

K.J.C. Leney ${ }^{144 b}$, T. Lenz ${ }^{104}$, G. Lenzen ${ }^{173}$, B. Lenzi ${ }^{29}$, K. Leonhardt ${ }^{43}$, S. Leontsinis ${ }^{9}$,

C. Leroy ${ }^{92}$, J-R. Lessard ${ }^{168}$, J. Lesser ${ }^{145 a}$, C.G. Lester ${ }^{27}$, A. Leung Fook Cheong ${ }^{171}$,

J. Levêque $^{4}$, D. Levin ${ }^{86}$, L.J. Levinson ${ }^{170}$, M.S. Levitski ${ }^{127}$, A. Lewis ${ }^{117}$, G.H. Lewis ${ }^{107}$,

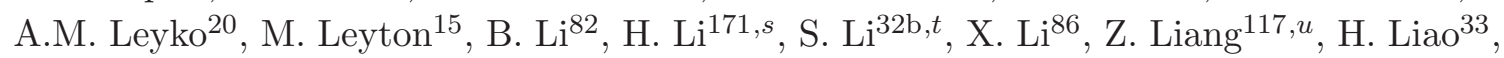

B. Liberti ${ }^{132 a}$, P. Lichard ${ }^{29}$, M. Lichtnecker ${ }^{97}$, K. Lie ${ }^{164}$, W. Liebig ${ }^{13}$, R. Lifshitz ${ }^{151}$,

C. $\operatorname{Limbach}^{20}$, A. Limosani ${ }^{85}$, M. Limper ${ }^{62}$, S.C. $\operatorname{Lin}^{150, v}$, F. Linde ${ }^{104}$, J.T. Linnemann ${ }^{87}$,

E. Lipeles ${ }^{119}$, L. Lipinsky ${ }^{124}$, A. Lipniacka ${ }^{13}$, T.M. Liss ${ }^{164}$, D. Lissauer ${ }^{24}$, A. Lister ${ }^{49}$,

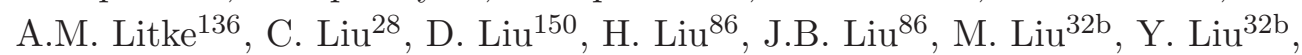

M. Livan ${ }^{118 a, 118 b}$, S.S.A. Livermore ${ }^{117}$, A. Lleres ${ }^{55}$, J. Llorente Merino ${ }^{79}$, S.L. Lloyd ${ }^{74}$,

E. Lobodzinska ${ }^{41}$, P. Loch $^{6}$, W.S. Lockman ${ }^{136}$, T. Loddenkoetter ${ }^{20}$, F.K. Loebinger ${ }^{81}$,

A. Loginov ${ }^{174}$, C.W. Loh ${ }^{167}$, T. Lohse ${ }^{15}$, K. Lohwasser ${ }^{48}$, M. Lokajicek ${ }^{124}$, J. Loken ${ }^{117}$, V.P. Lombardo ${ }^{4}$, R.E. Long ${ }^{70}$, L. Lopes ${ }^{123 a}$, D. Lopez Mateos ${ }^{57}$, J. Lorenz ${ }^{97}$,

N. Lorenzo Martinez ${ }^{114}$, M. Losada ${ }^{161}$, P. Loscutoff ${ }^{14}$, F. Lo Sterzo ${ }^{131 a, 131 b}$,

M.J. Losty ${ }^{158 a}$, X. Lou ${ }^{40}$, A. Lounis ${ }^{114}$, K.F. Loureiro ${ }^{161}$, J. Love ${ }^{21}$, P.A. Love ${ }^{70}$,

A.J. Lowe ${ }^{142, e}$, F. Lu ${ }^{32 a}$, H.J. Lubatti ${ }^{137}$, C. Luci ${ }^{131 a, 131 b}$, A. Lucotte ${ }^{55}$, A. Ludwig ${ }^{43}$,

D. Ludwig ${ }^{41}$, I. Ludwig ${ }^{48}$, J. Ludwig ${ }^{48}$, F. Luehring ${ }^{60}$, G. Luijckx ${ }^{104}$, D. Lumb ${ }^{48}$,

L. Luminari ${ }^{131 a}$, E. Lund ${ }^{116}$, B. Lund-Jensen ${ }^{146}$, B. Lundberg ${ }^{78}$, J. Lundberg ${ }^{145 a, 145 b}$,

J. Lundquist ${ }^{35}$, M. Lungwitz ${ }^{80}$, G. Lutz ${ }^{98}$, D. Lynn ${ }^{24}$, J. Lys ${ }^{14}$, E. Lytken ${ }^{78}$, H. Ma ${ }^{24}$, L.L. Ma ${ }^{171}$, J.A. Macana Goia ${ }^{92}$, G. Maccarrone ${ }^{47}$, A. Macchiolo ${ }^{98}$, B. Maček ${ }^{73}$,

J. Machado Miguens ${ }^{123 a}$, R. Mackeprang ${ }^{35}$, R.J. Madaras ${ }^{14}$, W.F. Mader ${ }^{43}$,

R. Maenner ${ }^{58 c}$, T. Maeno ${ }^{24}$, P. Mättig ${ }^{173}$, S. Mättig ${ }^{41}$, L. Magnoni ${ }^{29}$, E. Magradze ${ }^{54}$,

Y. Mahalalel ${ }^{152}$, K. Mahboubi ${ }^{48}$, G. Mahout ${ }^{17}$, C. Maiani ${ }^{131 a, 131 b}$, C. Maidantchik ${ }^{23 a}$,

A. Maio ${ }^{123 a, b}$, S. Majewski ${ }^{24}$, Y. Makida ${ }^{65}$, N. Makovec ${ }^{114}$, P. Mal ${ }^{135}$, B. Malaescu ${ }^{29}$,

Pa. Malecki ${ }^{38}$, P. Malecki ${ }^{38}$, V.P. Maleev ${ }^{120}$, F. Malek ${ }^{55}$, U. Mallik ${ }^{62}$, D. Malon ${ }^{5}$,

C. Malone ${ }^{142}$, S. Maltezos ${ }^{9}$, V. Malyshev ${ }^{106}$, S. Malyukov ${ }^{29}$, R. Mameghani ${ }^{97}$,

J. Mamuzic ${ }^{12 b}$, A. Manabe ${ }^{65}$, L. Mandelli ${ }^{88 a}$, I. Mandić ${ }^{73}$, R. Mandrysch ${ }^{15}$,

J. Maneira ${ }^{123 a}$, P.S. Mangeard ${ }^{87}$, L. Manhaes de Andrade Filho ${ }^{23 a}$, I.D. Manjavidze ${ }^{64}$,

A. Mann ${ }^{54}$, P.M. Manning ${ }^{136}$, A. Manousakis-Katsikakis ${ }^{8}$, B. Mansoulie ${ }^{135}$, A. Manz ${ }^{98}$, 
A. Mapelli ${ }^{29}$, L. Mapelli ${ }^{29}$, L. March ${ }^{79}$, J.F. Marchand ${ }^{28}$, F. Marchese ${ }^{132 a, 132 b}$, G. Marchiori ${ }^{77}$, M. Marcisovsky ${ }^{124}$, C.P. Marino ${ }^{168}$, F. Marroquim ${ }^{23 a}$, R. Marshall ${ }^{81}$, Z. Marshall ${ }^{29}$, F.K. Martens ${ }^{157}$, S. Marti-Garcia ${ }^{166}$, A.J. Martin ${ }^{174}$, B. Martin ${ }^{29}$,

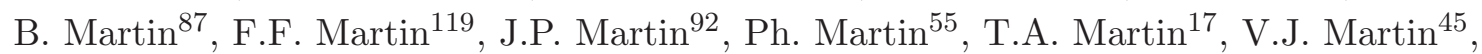
B. Martin dit Latour ${ }^{49}$, S. Martin-Haugh ${ }^{148}$, M. Martinez ${ }^{11}$, V. Martinez Outschoorn ${ }^{57}$, A.C. Martyniuk ${ }^{168}$, M. Marx ${ }^{81}$, F. Marzano ${ }^{131 a}$, A. Marzin ${ }^{110}$, L. Masetti ${ }^{80}$, T. Mashimo ${ }^{154}$, R. Mashinistov ${ }^{93}$, J. Masik ${ }^{81}$, A.L. Maslennikov ${ }^{106}$, I. Massa ${ }^{19 a, 19 b}$, G. Massaro ${ }^{104}$, N. Massol ${ }^{4}$, P. Mastrandrea ${ }^{131 a, 131 b}$, A. Mastroberardino ${ }^{36 a, 36 b}$, T. Masubuchi ${ }^{154}$, P. Matricon ${ }^{114}$, H. Matsumoto ${ }^{154}$, H. Matsunaga ${ }^{154}$, T. Matsushita ${ }^{66}$, C. Mattravers ${ }^{117, c}$, J.M. Maugain ${ }^{29}$, J. Maurer ${ }^{82}$, S.J. Maxfield ${ }^{72}$, D.A. Maximov ${ }^{106, f}$, E.N. May ${ }^{5}$, A. Mayne ${ }^{138}$, R. Mazini ${ }^{150}$, M. Mazur ${ }^{20}$, M. Mazzanti ${ }^{88 a}$, S.P. Mc Kee ${ }^{86}$, A. $\mathrm{McCarn}^{164}$, R.L. McCarthy ${ }^{147}$, T.G. McCarthy ${ }^{28}$, N.A. McCubbin ${ }^{128}$, K.W. McFarlane ${ }^{56}$, J.A. Mcfayden ${ }^{138}$, H. McGlone ${ }^{53}$, G. Mchedlidze ${ }^{51 b}$, R.A. McLaren ${ }^{29}$, T. Mclaughlan ${ }^{17}$, S.J. McMahon ${ }^{128}$, R.A. McPherson ${ }^{168, j}$, A. Meade ${ }^{83}$, J. Mechnich ${ }^{104}$, M. Mechtel ${ }^{173}$, M. Medinnis ${ }^{41}$, R. Meera-Lebbai ${ }^{110}$, T. Meguro ${ }^{115}$, R. Mehdiyev ${ }^{92}$, S. Mehlhase ${ }^{35}$, A. Mehta ${ }^{72}$, K. Meier ${ }^{58 a}$, B. Meirose ${ }^{78}$, C. Melachrinos ${ }^{30}$, B.R. Mellado Garcia ${ }^{171}$, L. Mendoza Navas ${ }^{161}$, Z. Meng ${ }^{150, s}$, A. Mengarelli ${ }^{19 a, 19 b}$, S. Menke ${ }^{98}$, C. Menot ${ }^{29}$, E. Meoni ${ }^{11}$, K.M. Mercurio ${ }^{57}$, P. Mermod ${ }^{49}$, L. Merola ${ }^{101 a, 101 b}$, C. Meroni ${ }^{88 a}$, F.S. Merritt ${ }^{30}$, H. Merritt ${ }^{108}$, A. Messina ${ }^{29}$, J. Metcalfe ${ }^{102}$, A.S. Mete ${ }^{63}$, C. Meyer $^{80}$, C. Meyer ${ }^{30}$, J-P. Meyer ${ }^{135}$, J. Meyer ${ }^{172}$, J. Meyer ${ }^{54}$, T.C. Meyer ${ }^{29}$, W.T. Meyer ${ }^{63}$, J. Miao ${ }^{32 d}$, S. Michal ${ }^{29}$, L. Micu ${ }^{25 a}$, R.P. Middleton ${ }^{128}$, S. Migas ${ }^{72}$, L. Mijović ${ }^{41}$, G. Mikenberg ${ }^{170}$, M. Mikestikova ${ }^{124}$, M. Mikuž ${ }^{73}$, D.W. Miller ${ }^{30}$, R.J. Miller ${ }^{87}$, W.J. Mills ${ }^{167}$, C. Mills ${ }^{57}$, A. Milov ${ }^{170}$, D.A. Milstead ${ }^{145 a, 145 b}$, D. Milstein ${ }^{170}$, A.A. Minaenko ${ }^{127}$, M. Miñano Moya ${ }^{166}$, I.A. Minashvili ${ }^{64}$, A.I. Mincer ${ }^{107}$, B. Mindur ${ }^{37}$, M. Mineev ${ }^{64}$, Y. Ming ${ }^{171}$, L.M. Mir ${ }^{11}$, G. Mirabelli131a, L. Miralles Verge ${ }^{11}$, A. Misiejuk ${ }^{75}$, J. Mitrevski ${ }^{136}$, G.Y. Mitrofanov ${ }^{127}$, V.A. Mitsou ${ }^{166}$, S. Mitsui ${ }^{65}$, P.S. Miyagawa ${ }^{138}$, K. Miyazaki ${ }^{66}$, J.U. Mjörnmark ${ }^{78}$, T. Moa ${ }^{145 a, 145 b}$, P. Mockett ${ }^{137}$, S. Moed ${ }^{57}$, V. Moeller ${ }^{27}$, K. Mönig ${ }^{41}$, N. Möser ${ }^{20}$, S. Mohapatra ${ }^{147}$, W. Mohr ${ }^{48}$, S. Mohrdieck-Möck ${ }^{98}$, A.M. Moisseev ${ }^{127, *}$, R. Moles-Valls ${ }^{166}$, J. Molina-Perez ${ }^{29}$, J. Monk ${ }^{76}$, E. Monnier ${ }^{82}$, S. Montesano ${ }^{88 a, 88 b}$, F. Monticelli69 ${ }^{69}$ S. Monzani ${ }^{19 a, 19 b}$, R.W. Moore ${ }^{2}$, G.F. Moorhead ${ }^{85}$, C. Mora Herrera ${ }^{49}$, A. Moraes ${ }^{53}$, N. Morange ${ }^{135}$, J. Morel ${ }^{54}$, G. Morello ${ }^{36 a, 36 b}$, D. Moreno ${ }^{80}$, M. Moreno Llácer ${ }^{166}$, P. Morettini ${ }^{50 a}$, M. Morgenstern ${ }^{43}$, M. Morii ${ }^{57}$, J. Morin ${ }^{74}$, A.K. Morley ${ }^{29}$, G. Mornacchi ${ }^{29}$,

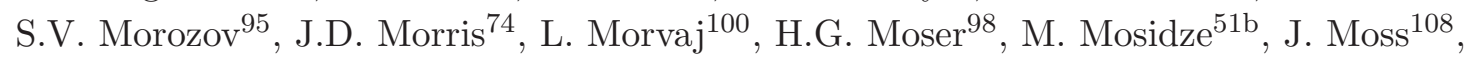
R. Mount ${ }^{142}$, E. Mountricha ${ }^{9, w}$, S.V. Mouraviev ${ }^{93}$, E.J.W. Moyse ${ }^{83}$, M. Mudrinic $^{12 b}$, F. Mueller ${ }^{58 a}$, J. Mueller ${ }^{122}$, K. Mueller ${ }^{20}$, T.A. Müller ${ }^{97}$, T. Mueller ${ }^{80}$, D. Muenstermann ${ }^{29}$, A. Muir ${ }^{167}$, Y. Munwes ${ }^{152}$, W.J. Murray ${ }^{128}$, I. Mussche ${ }^{104}$, E. Musto ${ }^{101 a, 101 b}$, A.G. Myagkov ${ }^{127}$, M. Myska ${ }^{124}$, J. Nadal ${ }^{11}$, K. Nagai ${ }^{159}$, K. Nagano ${ }^{65}$, A. Nagarkar ${ }^{108}$, Y. Nagasaka ${ }^{59}$, M. Nagel ${ }^{98}$, A.M. Nairz ${ }^{29}$, Y. Nakahama ${ }^{29}$, K. Nakamura ${ }^{154}$, T. Nakamura ${ }^{154}$, I. Nakano ${ }^{109}$, G. Nanava ${ }^{20}$, A. Napier ${ }^{160}$, R. Narayan ${ }^{58 b}$, M. Nash ${ }^{76, c}$, N.R. Nation ${ }^{21}$, T. Nattermann ${ }^{20}$, T. Naumann ${ }^{41}$, G. Navarro ${ }^{161}$, H.A. Neal ${ }^{86}$, E. Nebot ${ }^{79}$, P.Yu. Nechaeva ${ }^{93}$, T.J. Neep ${ }^{81}$, A. Negri ${ }^{118 a, 118 b}$, G. Negri ${ }^{29}$, S. Nektarijevic ${ }^{49}$, A. Nelson ${ }^{162}$, S. Nelson ${ }^{142}$, T.K. Nelson ${ }^{142}$, S. Nemecek ${ }^{124}$, 
P. Nemethy ${ }^{107}$, A.A. Nepomuceno ${ }^{23 a}$, M. Nessi ${ }^{29, x}$, M.S. Neubauer ${ }^{164}$, A. Neusiedl ${ }^{80}$, R.M. Neves ${ }^{107}$, P. Nevski ${ }^{24}$, P.R. Newman ${ }^{17}$, V. Nguyen Thi Hong ${ }^{135}$, R.B. Nickerson ${ }^{117}$, R. Nicolaidou ${ }^{135}$, L. Nicolas ${ }^{138}$, B. Nicquevert ${ }^{29}$, F. Niedercorn ${ }^{114}$, J. Nielsen ${ }^{136}$, T. Niinikoski ${ }^{29}$, N. Nikiforou ${ }^{34}$, A. Nikiforov ${ }^{15}$, V. Nikolaenko ${ }^{127}$, K. Nikolaev ${ }^{64}$, I. Nikolic-Audit ${ }^{77}$, K. Nikolics ${ }^{49}$, K. Nikolopoulos ${ }^{24}$, H. Nilsen ${ }^{48}$, P. Nilsson ${ }^{7}$, Y. Ninomiya ${ }^{154}$, A. Nisati ${ }^{131 a}$, T. Nishiyama ${ }^{66}$, R. Nisius $^{98}$, L. Nodulman ${ }^{5}$, M. Nomachi ${ }^{115}$, I. Nomidis ${ }^{153}$, M. Nordberg ${ }^{29}$, B. Nordkvist ${ }^{145 a, 145 b}$, P.R. Norton ${ }^{128}$, J. Novakova ${ }^{125}$, M. Nozaki ${ }^{65}$, L. Nozka ${ }^{112}$, I.M. Nugent ${ }^{158 a}$, A.-E. Nuncio-Quiroz ${ }^{20}$, G. Nunes Hanninger ${ }^{85}$, T. Nunnemann ${ }^{97}$, E. Nurse ${ }^{76}$, B.J. O'Brien ${ }^{45}$, S.W. O'Neale ${ }^{17, *}$, D.C. O'Neil ${ }^{141}$, V. O'Shea ${ }^{53}$, L.B. Oakes ${ }^{97}$, F.G. Oakham ${ }^{28, d}$, H. Oberlack ${ }^{98}$, J. Ocariz ${ }^{77}$,

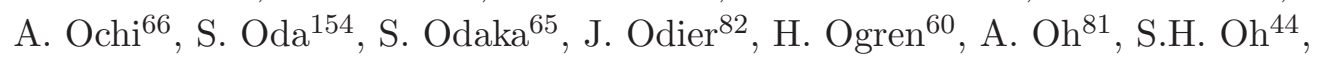
C.C. Ohm $^{145 a, 145 b}$, T. Ohshima ${ }^{100}$, H. Ohshita ${ }^{139}$, T. Ohsugi ${ }^{177}$, S. Okada ${ }^{66}$, H. Okawa ${ }^{162}$, Y. Okumura ${ }^{100}$, T. Okuyama ${ }^{154}$, A. Olariu ${ }^{25 a}$, M. Olcese ${ }^{50 a}$, A.G. Olchevski ${ }^{64}$, S.A. Olivares Pino ${ }^{31 a}$, M. Oliveira ${ }^{123 a, h}$, D. Oliveira Damazio ${ }^{24}$, E. Oliver Garcia ${ }^{166}$, D. Olivito ${ }^{119}$, A. Olszewski ${ }^{38}$, J. Olszowska ${ }^{38}$, C. Omachi ${ }^{66}$, A. Onofre ${ }^{123 a, y}$, P.U.E. Onyisi ${ }^{30}$, C.J. Oram ${ }^{158 a}$, M.J. Oreglia ${ }^{30}$, Y. Oren ${ }^{152}$, D. Orestano ${ }^{133 a, 133 b}$, I. Orlov ${ }^{106}$, C. Oropeza Barrera ${ }^{53}$, R.S. Orr ${ }^{157}$, B. Osculati50a,50b, R. Ospanov ${ }^{119}$, C. Osuna ${ }^{11}$, G. Otero y Garzon ${ }^{26}$, J.P. Ottersbach ${ }^{104}$, M. Ouchrif ${ }^{134 d}$, E.A. Ouellette ${ }^{168}$, F. Ould-Saada ${ }^{116}$, A. Ouraou ${ }^{135}$, Q. Ouyang ${ }^{32 a}$, A. Ovcharova ${ }^{14}$, M. Owen ${ }^{81}$, S. Owen ${ }^{138}$, V.E. Ozcan ${ }^{18 a}$, N. Ozturk ${ }^{7}$, A. Pacheco Pages ${ }^{11}$,

C. Padilla Aranda ${ }^{11}$, S. Pagan Griso ${ }^{14}$, E. Paganis ${ }^{138}$, F. Paige ${ }^{24}$, P. Pais $^{83}$, K. Pajchel $^{116}$, G. Palacino ${ }^{158 b}$, C.P. Paleari ${ }^{6}$, S. Palestini ${ }^{29}$, D. Pallin ${ }^{33}$, A. Palma ${ }^{123 a}$, J.D. Palmer ${ }^{17}$, Y.B. Pan ${ }^{171}$, E. Panagiotopoulou ${ }^{9}$, B. Panes ${ }^{31 a}$, N. Panikashvili ${ }^{86}$, S. Panitkin ${ }^{24}$, D. Pantea ${ }^{25 a}$, M. Panuskova ${ }^{124}$, V. Paolone ${ }^{122}$, A. Papadelis ${ }^{145 a}$, Th.D. Papadopoulou ${ }^{9}$, A. Paramonov ${ }^{5}$, D. Paredes Hernandez ${ }^{33}$, W. $\operatorname{Park}^{24, z}$, M.A. Parker ${ }^{27}$, F. Parodi ${ }^{50 a, 50 b}$, J.A. Parsons ${ }^{34}$, U. Parzefall ${ }^{48}$, E. Pasqualucci ${ }^{131 a}$, S. Passaggio ${ }^{50 a}$, A. Passeri133a, F. Pastore ${ }^{133 a, 133 b}$, Fr. Pastore ${ }^{75}$, G. Pásztor ${ }^{49, a a}$, S. Pataraia ${ }^{173}$, N. Patel ${ }^{149}$, J.R. Pater ${ }^{81}$, S. Patricelli ${ }^{101 a, 101 b}$, T. Pauly ${ }^{29}$, M. Pecsy ${ }^{143 a}$, M.I. Pedraza Morales ${ }^{171}$, S.V. Peleganchuk ${ }^{106}$, H. Peng ${ }^{32 b}$, R. Pengo ${ }^{29}$, B. Penning ${ }^{30}$, A. Penson ${ }^{34}$, J. Penwell ${ }^{60}$, M. Perantoni ${ }^{23 a}$, K. Perez ${ }^{34, a b}$, T. Perez Cavalcanti ${ }^{41}$, E. Perez Codina ${ }^{11}$, M.T. Pérez García-Estañ $^{166}$, V. Perez Reale ${ }^{34}$, L. Perini ${ }^{88 a, 88 b}$, H. Pernegger ${ }^{29}$, R. Perrino ${ }^{71 a}$, P. Perrodo ${ }^{4}$, S. Persembe ${ }^{3 a}$, A. Perus ${ }^{114}$, V.D. Peshekhonov ${ }^{64}$, K. Peters ${ }^{29}$,

B.A. Petersen ${ }^{29}$, J. Petersen ${ }^{29}$, T.C. Petersen ${ }^{35}$, E. Petit ${ }^{4}$, A. Petridis ${ }^{153}$, C. Petridou ${ }^{153}$, E. Petrolo ${ }^{131 a}$, F. Petrucci ${ }^{133 a, 133 b}$, D. Petschull ${ }^{41}$, M. Petteni ${ }^{141}$, R. Pezoa ${ }^{31 b}$, A. Phan $^{85}$, P.W. Phillips ${ }^{128}$, G. Piacquadio ${ }^{29}$, A. Picazio ${ }^{49}$, E. Piccaro ${ }^{74}$, M. Piccinini ${ }^{19 a, 19 b}$, S.M. Piec $^{41}$, R. Piegaia ${ }^{26}$, D.T. Pignotti ${ }^{108}$, J.E. Pilcher ${ }^{30}$, A.D. Pilkington ${ }^{81}$, J. Pina ${ }^{123 a}, b$, M. Pinamonti163a,163c , A. Pinder ${ }^{117}$, J.L. Pinfold ${ }^{2}$, J. Ping ${ }^{32 c}$, B. Pinto ${ }^{123 a}$, O. Pirotte ${ }^{29}$, C. Pizio ${ }^{88 a, 88 b}$, M. Plamondon ${ }^{168}$, M.-A. Pleier ${ }^{24}$, A.V. Pleskach ${ }^{127}$,

A. Poblaguev ${ }^{24}$, S. Poddar ${ }^{58 a}$, F. Podlyski ${ }^{33}$, L. Poggioli ${ }^{114}$, T. Poghosyan ${ }^{20}$, M. Pohl ${ }^{49}$, F. Polci ${ }^{55}$, G. Polesello ${ }^{118 a}$, A. Policicchio ${ }^{36 a, 36 b}$, A. Polini ${ }^{19 a}$, J. Poll $^{74}$,

V. Polychronakos ${ }^{24}$, D.M. Pomarede ${ }^{135}$, D. Pomeroy ${ }^{22}$, K. Pommès ${ }^{29}$, L. Pontecorvo $^{131 a}$, B.G. Pope ${ }^{87}$, G.A. Popeneciu ${ }^{25 a}$, D.S. Popovic ${ }^{12 a}$, A. Poppleton ${ }^{29}$, X. Portell Bueso ${ }^{29}$, C. Posch $^{21}$, G.E. Pospelov ${ }^{98}$, S. Pospisil ${ }^{126}$, I.N. Potrap ${ }^{98}$, C.J. Potter ${ }^{148}$, C.T. Potter ${ }^{113}$, 
G. Poulard ${ }^{29}$, J. Poveda ${ }^{171}$, V. Pozdnyakov ${ }^{64}$, R. Prabhu ${ }^{76}$, P. Pralavorio ${ }^{82}$, A. Pranko ${ }^{14}$,

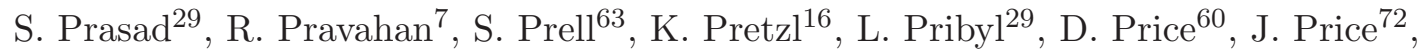
L.E. Price ${ }^{5}$, M.J. Price ${ }^{29}$, D. Prieur ${ }^{122}$, M. Primavera ${ }^{71 a}$, K. Prokofiev ${ }^{107}$, F. Prokoshin ${ }^{31 b}$, S. Protopopescu ${ }^{24}$, J. Proudfoot ${ }^{5}$, X. Prudent ${ }^{43}$, M. Przybycien ${ }^{37}$, H. Przysiezniak ${ }^{4}$, S. Psoroulas ${ }^{20}$, E. Ptacek ${ }^{113}$, E. Pueschel ${ }^{83}$, J. Purdham ${ }^{86}$, M. Purohit ${ }^{24, z}$, P. Puzo ${ }^{114}$, Y. Pylypchenko ${ }^{62}$, J. Qian ${ }^{86}$, Z. Qian ${ }^{82}$, Z. Qin ${ }^{41}$, A. Quadt ${ }^{54}$,

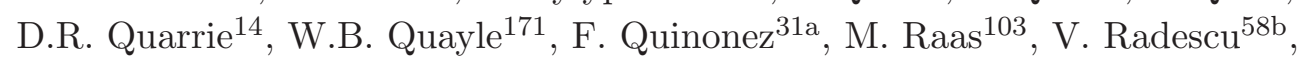

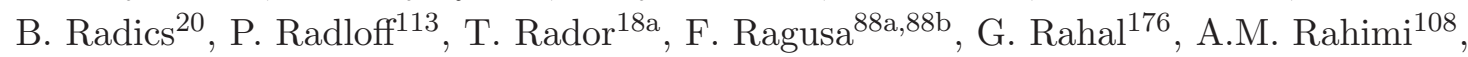
D. Rahm ${ }^{24}$, S. Rajagopalan ${ }^{24}$, M. Rammensee ${ }^{48}$, M. Rammes ${ }^{140}$, A.S. Randle-Conde ${ }^{39}$, K. Randrianarivony ${ }^{28}$, P.N. Ratoff ${ }^{70}$, F. Rauscher ${ }^{97}$, T.C. Rave ${ }^{48}$, M. Raymond ${ }^{29}$, A.L. Read ${ }^{116}$, D.M. Rebuzzi 118a,118b, A. Redelbach ${ }^{172}$, G. Redlinger ${ }^{24}$, R. Reece $^{119}$, K. Reeves ${ }^{40}$, A. Reichold ${ }^{104}$, E. Reinherz-Aronis ${ }^{152}$, A. Reinsch ${ }^{113}$, I. Reisinger ${ }^{42}$, C. Rembser ${ }^{29}$, Z.L. Ren ${ }^{150}$, A. Renaud ${ }^{114}$, M. Rescigno ${ }^{131 a}$, S. Resconi ${ }^{88 a}$, B. Resende ${ }^{135}$, P. Reznicek ${ }^{97}$, R. Rezvani ${ }^{157}$, A. Richards ${ }^{76}$, R. Richter ${ }^{98}$, E. Richter-Was ${ }^{4, a c}$, M. Ridel $^{77}$, M. Rijpstra ${ }^{104}$, M. Rijssenbeek ${ }^{147}$, A. Rimoldi ${ }^{118 a, 118 b}$, L. Rinaldi ${ }^{19 a}$, R.R. Rios ${ }^{39}$, I. Riu ${ }^{11}$, G. Rivoltella ${ }^{88 a, 88 b}$, F. Rizatdinova ${ }^{111}$, E. Rizvi ${ }^{74}$, S.H. Robertson ${ }^{84, j}$, A. Robichaud-Veronneau ${ }^{117}$, D. Robinson ${ }^{27}$, J.E.M. Robinson ${ }^{76}$, A. Robson ${ }^{53}$, J.G. Rocha de Lima ${ }^{105}$, C. Roda ${ }^{121 a, 121 b}$, D. Roda Dos Santos ${ }^{29}$, D. Rodriguez ${ }^{161}$, A. $\operatorname{Roe}^{54}$, S. Roe ${ }^{29}$, O. Røhne ${ }^{116}$, V. Rojo ${ }^{1}$, S. Rolli ${ }^{160}$, A. Romaniouk ${ }^{95}$, M. Romano ${ }^{19 a, 19 b}$, V.M. Romanov ${ }^{64}$, G. Romeo ${ }^{26}$, E. Romero Adam ${ }^{166}$, L. Roos ${ }^{77}$, E. $R^{16 s}{ }^{166}$, S. Rosati ${ }^{131 a}$, K. Rosbach ${ }^{49}$, A. Rose ${ }^{148}$, M. $\operatorname{Rose}^{75}$, G.A. Rosenbaum ${ }^{157}$, E.I. Rosenberg ${ }^{63}$, P.L. Rosendahl ${ }^{13}$, O. Rosenthal ${ }^{140}$, L. Rosselet ${ }^{49}$, V. Rossetti ${ }^{11}$, E. Rossi ${ }^{131 a, 131 b}$, L.P. Rossi ${ }^{50 a}$, M. Rotaru ${ }^{25 a}$, I. Roth ${ }^{170}$, J. Rothberg ${ }^{137}$, D. Rousseau ${ }^{114}$, C.R. Royon ${ }^{135}$, A. Rozanov ${ }^{82}$, Y. Rozen ${ }^{151}$, X. Ruan ${ }^{32 a, a d}$, I. Rubinskiy ${ }^{41}$, B. Ruckert ${ }^{97}$, N. Ruckstuhl ${ }^{104}$, V.I. Rud ${ }^{96}$, C. Rudolph ${ }^{43}$, G. Rudolph ${ }^{61}$, F. Rühr ${ }^{6}$, F. Ruggieri133a,133b, A. Ruiz-Martinez ${ }^{63}$, V. Rumiantsev ${ }^{90, *}$, L. Rumyantsev ${ }^{64}$, K. Runge ${ }^{48}$, Z. Rurikova $^{48}$, N.A. Rusakovich ${ }^{64}$, J.P. Rutherfoord ${ }^{6}$, C. Ruwiedel ${ }^{14}$, P. Ruzicka ${ }^{124}$, Y.F. Ryabov ${ }^{120}$, V. Ryadovikov ${ }^{127}$, P. Ryan ${ }^{87}$, M. Rybar ${ }^{125}$, G. Rybkin ${ }^{114}$, N.C. Ryder ${ }^{117}$, S. Rzaeva ${ }^{10}$, A.F. Saavedra ${ }^{149}$, I. Sadeh ${ }^{152}$, H.F-W. Sadrozinski ${ }^{136}$, R. Sadykov ${ }^{64}$, F. Safai Tehrani ${ }^{131 a}$, H. Sakamoto ${ }^{154}$, G. Salamanna ${ }^{74}$, A. Salamon ${ }^{132 a}$, M. Saleem ${ }^{110}$, D. Salihagic ${ }^{98}$,

A. Salnikov ${ }^{142}$, J. Salt ${ }^{166}$, B.M. Salvachua Ferrando ${ }^{5}$, D. Salvatore ${ }^{36 a, 36 b}$, F. Salvatore ${ }^{148}$, A. Salvucci ${ }^{103}$, A. Salzburger ${ }^{29}$, D. Sampsonidis ${ }^{153}$, B.H. Samset ${ }^{116}$, A. Sanchez ${ }^{101 a, 101 b}$, V. Sanchez Martinez ${ }^{166}$, H. Sandaker ${ }^{13}$, H.G. Sander ${ }^{80}$, M.P. Sanders ${ }^{97}$, M. Sandhoff ${ }^{173}$, T. Sandoval ${ }^{27}$, C. Sandoval ${ }^{161}$, R. Sandstroem ${ }^{98}$, S. Sandvoss ${ }^{173}$, D.P.C. Sankey ${ }^{128}$, A. Sansoni ${ }^{47}$, C. Santamarina Rios ${ }^{84}$, C. Santoni ${ }^{33}$, R. Santonico ${ }^{132 a, 132 b}$, H. Santos ${ }^{123 a}$, J.G. Saraiva ${ }^{123 a}$, T. Sarangi ${ }^{171}$, E. Sarkisyan-Grinbaum ${ }^{7}$, F. Sarri ${ }^{121 a, 121 b}$,

G. Sartisohn ${ }^{173}$, O. Sasaki ${ }^{65}$, N. Sasao ${ }^{67}$, I. Satsounkevitch ${ }^{89}$, G. Sauvage ${ }^{4}$, E. Sauvan ${ }^{4}$, J.B. Sauvan ${ }^{114}$, P. Savard ${ }^{157, d}$, V. Savinov ${ }^{122}$, D.O. Savu ${ }^{29}$, L. Sawyer ${ }^{24, l}$, D.H. Saxon ${ }^{53}$, L.P. Says ${ }^{33}$, C. Sbarra ${ }^{19 a}$, A. Sbrizzi ${ }^{19 a, 19 b}$, O. Scallon ${ }^{92}$, D.A. Scannicchio ${ }^{162}$, M. Scarcella ${ }^{149}$, J. Schaarschmidt ${ }^{114}$, P. Schacht ${ }^{98}$, U. Schäfer ${ }^{80}$, S. Schaepe ${ }^{20}$, S. Schaetzel ${ }^{58 b}$, A.C. Schaffer ${ }^{114}$, D. Schaile ${ }^{97}$, R.D. Schamberger ${ }^{147}$, A.G. Schamov ${ }^{106}$, V. Scharf ${ }^{58 a}$, V.A. Schegelsky ${ }^{120}$, D. Scheirich ${ }^{86}$, M. Schernau ${ }^{162}$, M.I. Scherzer ${ }^{34}$, C. Schiavi ${ }^{50 a, 50 b}$, J. Schieck ${ }^{97}$, M. Schioppa ${ }^{36 a, 36 b}$, S. Schlenker ${ }^{29}$, J.L. Schlereth ${ }^{5}$, 


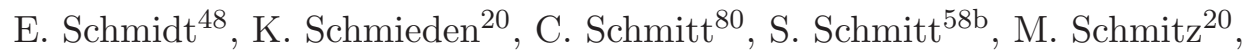

A. Schöning ${ }^{58 b}$, M. Schott ${ }^{29}$, D. Schouten ${ }^{158 a}$, J. Schovancova ${ }^{124}$, M. Schram ${ }^{84}$,

C. Schroeder ${ }^{80}$, N. Schroer ${ }^{58 c}$, G. Schuler ${ }^{29}$, M.J. Schultens ${ }^{20}$, J. Schultes ${ }^{173}$,

H.-C. Schultz-Coulon ${ }^{58 a}$, H. Schulz ${ }^{15}$, J.W. Schumacher ${ }^{20}$, M. Schumacher ${ }^{48}$,

B.A. Schumm ${ }^{136}$, Ph. Schune ${ }^{135}$, C. Schwanenberger ${ }^{81}$, A. Schwartzman ${ }^{142}$,

Ph. Schwemling ${ }^{77}$, R. Schwienhorst ${ }^{87}$, R. Schwierz ${ }^{43}$, J. Schwindling ${ }^{135}$, T. Schwindt ${ }^{20}$,

M. Schwoerer ${ }^{4}$, W.G. Scott ${ }^{128}$, J. Searcy ${ }^{113}$, G. Sedov ${ }^{41}$, E. Sedykh ${ }^{120}$, E. Segura ${ }^{11}$,

S.C. Seidel ${ }^{102}$, A. Seiden ${ }^{136}$, F. Seifert ${ }^{43}$, J.M. Seixas ${ }^{23 a}$, G. Sekhniaidze ${ }^{101 a}$,

K.E. Selbach ${ }^{45}$, D.M. Seliverstov ${ }^{120}$, B. Sellden ${ }^{145 a}$, G. Sellers ${ }^{72}$, M. Seman ${ }^{143 b}$,

N. Semprini-Cesari ${ }^{19 a, 19 b}$, C. Serfon ${ }^{97}$, L. Serin ${ }^{114}$, L. Serkin ${ }^{54}$, R. Seuster ${ }^{98}$,

H. Severini ${ }^{110}$, M.E. Sevior ${ }^{85}$, A. Sfyrla ${ }^{29}$, E. Shabalina ${ }^{54}$, M. Shamim ${ }^{113}$, L.Y. Shan ${ }^{32 a}$, J.T. Shank ${ }^{21}$, Q.T. Shao ${ }^{85}$, M. Shapiro ${ }^{14}$, P.B. Shatalov ${ }^{94}$, L. Shaver ${ }^{6}$, K. Shaw ${ }^{163 a, 163 c}$, D. Sherman ${ }^{174}$, P. Sherwood ${ }^{76}$, A. Shibata ${ }^{107}$, H. Shichi ${ }^{100}$, S. Shimizu ${ }^{29}$,

M. Shimojima ${ }^{99}$, T. Shin ${ }^{56}$, M. Shiyakova ${ }^{64}$, A. Shmeleva ${ }^{93}$, M.J. Shochet ${ }^{30}$, D. Short ${ }^{117}$, S. Shrestha ${ }^{63}$, E. Shulga ${ }^{95}$, M.A. Shupe ${ }^{6}$, P. Sicho ${ }^{124}$, A. Sidoti ${ }^{131 a}$, F. Siegert ${ }^{48}$,

Dj. Sijacki ${ }^{12 a}$, O. Silbert ${ }^{170}$, J. Silva ${ }^{123 a}$, Y. Silver ${ }^{152}$, D. Silverstein ${ }^{142}$,

S.B. Silverstein ${ }^{145 a}$, V. Simak ${ }^{126}$, O. Simard ${ }^{135}$, Lj. Simic ${ }^{12 a}$, S. Simion ${ }^{114}$, B. Simmons ${ }^{76}$, M. Simonyan ${ }^{35}$, P. Sinervo ${ }^{157}$, N.B. Sinev ${ }^{113}$, V. Sipica ${ }^{140}$, G. Siragusa ${ }^{172}$, A. Sircar ${ }^{24}$, A.N. Sisakyan ${ }^{64}$, S.Yu. Sivoklokov ${ }^{96}$, J. Sjölin ${ }^{145 a, 145 b}$, T.B. Sjursen ${ }^{13}$, L.A. Skinnari ${ }^{14}$, H.P. Skottowe ${ }^{57}$, K. Skovpen ${ }^{106}$, P. Skubic ${ }^{110}$, N. Skvorodnev ${ }^{22}$, M. Slater ${ }^{17}$,

T. Slavicek ${ }^{126}$, K. Sliwa ${ }^{160}$, J. Sloper ${ }^{29}$, V. Smakhtin ${ }^{170}$, B.H. Smart ${ }^{45}$, S.Yu. Smirnov ${ }^{95}$,

Y. Smirnov ${ }^{95}$, L.N. Smirnova ${ }^{96}$, O. Smirnova ${ }^{78}$, B.C. Smith ${ }^{57}$, D. Smith ${ }^{142}$,

K.M. Smith ${ }^{53}$, M. Smizanska ${ }^{70}$, K. Smolek ${ }^{126}$, A.A. Snesarev ${ }^{93}$, S.W. Snow ${ }^{81}$, J. Snow ${ }^{110}$, J. Snuverink ${ }^{104}$, S. Snyder ${ }^{24}$, M. Soares ${ }^{123 a}$, R. Sobie ${ }^{168, j}$, J. Sodomka ${ }^{126}$, A. Soffer ${ }^{152}$, C.A. Solans ${ }^{166}$, M. Solar ${ }^{126}$, J. Solc ${ }^{126}$, E. Soldatov ${ }^{95}$, U. Soldevila ${ }^{166}$,

E. Solfaroli Camillocci131a,131b, A.A. Solodkov ${ }^{127}$, O.V. Solovyanov ${ }^{127}$, N. Soni ${ }^{2}$,

V. Sopko ${ }^{126}$, B. Sopko ${ }^{126}$, M. Sosebee ${ }^{7}$, R. Soualah ${ }^{163 a, 163 c}$, A. Soukharev ${ }^{106}$,

S. Spagnolo ${ }^{71 a, 71 b}$, F. Spanò ${ }^{75}$, R. Spighi ${ }^{19 a}$, G. Spigo ${ }^{29}$, F. Spila ${ }^{131 a, 131 b}$, R. Spiwoks ${ }^{29}$,

M. Spousta ${ }^{125}$, T. Spreitzer ${ }^{157}$, B. Spurlock ${ }^{7}$, R.D. St. Denis ${ }^{53}$, J. Stahlman ${ }^{119}$,

R. Stamen ${ }^{58 a}$, E. Stanecka ${ }^{38}$, R.W. Stanek ${ }^{5}$, C. Stanescu ${ }^{133 a}$, S. Stapnes ${ }^{116}$,

E.A. Starchenko ${ }^{127}$, J. Stark ${ }^{55}$, P. Staroba ${ }^{124}$, P. Starovoitov ${ }^{90}$, A. Staude ${ }^{97}$,

P. Stavina ${ }^{143 a}$, G. Steele ${ }^{53}$, P. Steinbach ${ }^{43}$, P. Steinberg ${ }^{24}$, I. Stekl ${ }^{126}$, B. Stelzer ${ }^{141}$,

H.J. Stelzer ${ }^{87}$, O. Stelzer-Chilton ${ }^{158 a}$, H. Stenzel ${ }^{52}$, S. Stern ${ }^{98}$, K. Stevenson ${ }^{74}$,

G.A. Stewart ${ }^{29}$, J.A. Stillings ${ }^{20}$, M.C. Stockton ${ }^{84}$, K. Stoerig ${ }^{48}$, G. Stoicea ${ }^{25 a}$,

S. Stonjek ${ }^{98}$, P. Strachota ${ }^{125}$, A.R. Stradling ${ }^{7}$, A. Straessner ${ }^{43}$, J. Strandberg ${ }^{146}$,

S. Strandberg ${ }^{145 a, 145 b}$, A. Strandlie ${ }^{116}$, M. Strang ${ }^{108}$, E. Strauss ${ }^{142}$, M. Strauss ${ }^{110}$,

P. Strizenec ${ }^{143 b}$, R. Ströhmer ${ }^{172}$, D.M. Strom ${ }^{113}$, J.A. Strong ${ }^{75, *}$, R. Stroynowski ${ }^{39}$,

J. Strube ${ }^{128}$, B. Stugu ${ }^{13}$, I. Stumer ${ }^{24, *}$, J. Stupak ${ }^{147}$, P. Sturm ${ }^{173}$, N.A. Styles ${ }^{41}$,

D.A. Soh ${ }^{150, u}$, D. Su ${ }^{142}$, HS. Subramania ${ }^{2}$, A. Succurro ${ }^{11}$, Y. Sugaya ${ }^{115}$, T. Sugimoto ${ }^{100}$,

C. Suhr ${ }^{105}$, K. Suita ${ }^{66}$, M. Suk ${ }^{125}$, V.V. Sulin ${ }^{93}$, S. Sultansoy ${ }^{3 d}$, T. Sumida ${ }^{67}$, X. Sun ${ }^{55}$,

J.E. Sundermann ${ }^{48}$, K. Suruliz ${ }^{138}$, S. Sushkov ${ }^{11}$, G. Susinno ${ }^{36 a, 36 b}$, M.R. Sutton ${ }^{148}$,

Y. Suzuki ${ }^{65}$, Y. Suzuki ${ }^{66}$, M. Svatos ${ }^{124}$, Yu.M. Sviridov ${ }^{127}$, S. Swedish ${ }^{167}$, I. Sykora ${ }^{143 a}$,

T. Sykora ${ }^{125}$, B. Szeless ${ }^{29}$, J. Sánchez ${ }^{166}$, D. Ta ${ }^{104}$, K. Tackmann ${ }^{41}$, A. Taffard ${ }^{162}$, 
R. Tafirout ${ }^{158 a}$, N. Taiblum ${ }^{152}$, Y. Takahashi ${ }^{100}$, H. Takai ${ }^{24}$, R. Takashima ${ }^{68}$, H. Takeda ${ }^{66}$, T. Takeshita ${ }^{139}$, Y. Takubo ${ }^{65}$, M. Talby ${ }^{82}$, A. Talyshev ${ }^{106, f}$, M.C. Tamsett ${ }^{24}$, J. Tanaka ${ }^{154}$, R. Tanaka ${ }^{114}$, S. Tanaka ${ }^{130}$, S. Tanaka ${ }^{65}$, Y. Tanaka ${ }^{99}$, A.J. Tanasijczuk ${ }^{141}$, K. Tani ${ }^{66}$, N. Tannoury ${ }^{82}$, G.P. Tappern ${ }^{29}$, S. Tapprogge ${ }^{80}$,

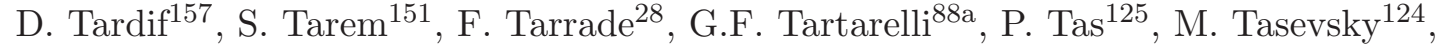
E. Tassi ${ }^{36 a, 36 b}$, M. Tatarkhanov ${ }^{14}$, Y. Tayalati ${ }^{134 d}$, C. Taylor ${ }^{76}$, F.E. Taylor ${ }^{91}$, G.N. Taylor ${ }^{85}$, W. Taylor ${ }^{158 b}$, M. Teinturier ${ }^{114}$, M. Teixeira Dias Castanheira ${ }^{74}$,

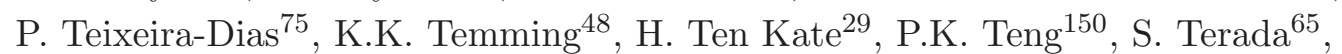
K. Terashi ${ }^{154}$, J. Terron ${ }^{79}$, M. Testa ${ }^{47}$, R.J. Teuscher ${ }^{157, j}$, J. Thadome ${ }^{173}$, J. Therhaag ${ }^{20}$, T. Theveneaux-Pelzer ${ }^{77}$, M. Thioye ${ }^{174}$, S. Thoma ${ }^{48}$, J.P. Thomas ${ }^{17}$, E.N. Thompson ${ }^{34}$, P.D. Thompson ${ }^{17}$, P.D. Thompson ${ }^{157}$, A.S. Thompson ${ }^{53}$, L.A. Thomsen ${ }^{35}$,

E. Thomson ${ }^{119}$, M. Thomson ${ }^{27}$, R.P. Thun ${ }^{86}$, F. $\operatorname{Tian}^{34}$, M.J. Tibbetts ${ }^{14}$, T. Tic ${ }^{124}$, V.O. Tikhomirov ${ }^{93}$, Y.A. Tikhonov ${ }^{106, f}$, S. Timoshenko ${ }^{95}$, P. Tipton ${ }^{174}$,

F.J. Tique Aires Viegas ${ }^{29}$, S. Tisserant ${ }^{82}$, B. Toczek ${ }^{37}$, T. Todorov ${ }^{4}$, S. Todorova-Nova ${ }^{160}$, B. Toggerson ${ }^{162}$, J. Tojo ${ }^{65}$, S. Tokár ${ }^{143 a}$, K. Tokunaga ${ }^{66}$, K. Tokushuku ${ }^{65}$, K. Tollefson ${ }^{87}$,

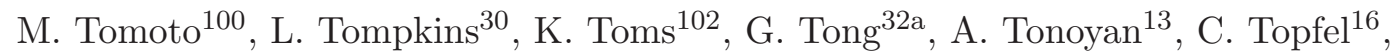
N.D. Topilin ${ }^{64}$, I. Torchiani ${ }^{29}$, E. Torrence ${ }^{113}$, H. Torres ${ }^{77}$, E. Torró Pastor ${ }^{166}$, J. Toth ${ }^{82, a a}$, F. Touchard ${ }^{82}$, D.R. Tovey ${ }^{138}$, T. Trefzger ${ }^{172}$, L. Tremblet ${ }^{29}$, A. Tricoli ${ }^{29}$, I.M. Trigger ${ }^{158 a}$, S. Trincaz-Duvoid ${ }^{77}$, T.N. Trinh ${ }^{77}$, M.F. Tripiana ${ }^{69}$, W. Trischuk ${ }^{157}$,

A. Trivedi ${ }^{24, z}$, B. Trocmé ${ }^{55}$, C. Troncon ${ }^{88 a}$, M. Trottier-McDonald ${ }^{141}$, M. Trzebinski ${ }^{38}$, A. Trzupek ${ }^{38}$, C. Tsarouchas ${ }^{29}$, J.C-L. Tseng ${ }^{117}$, M. Tsiakiris ${ }^{104}$, P.V. Tsiareshka ${ }^{89}$, D. Tsionou ${ }^{4, a e}$, G. Tsipolitis ${ }^{9}$, V. Tsiskaridze ${ }^{48}$, E.G. Tskhadadze ${ }^{51 a}$, I.I. Tsukerman ${ }^{94}$,

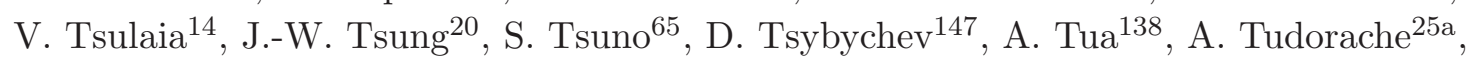
V. Tudorache ${ }^{25 a}$, J.M. Tuggle ${ }^{30}$, M. Turala ${ }^{38}$, D. Turecek ${ }^{126}$, I. Turk Cakir ${ }^{3 e}$,

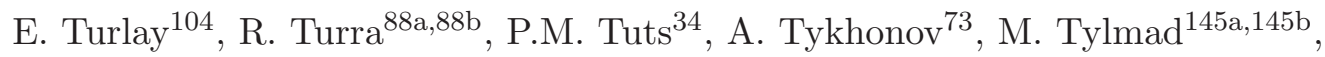

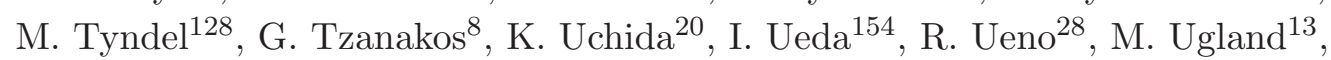
M. Uhlenbrock ${ }^{20}$, M. Uhrmacher ${ }^{54}$, F. Ukegawa ${ }^{159}$, G. Unal ${ }^{29}$, D.G. Underwood ${ }^{5}$, A. Undrus ${ }^{24}$, G. Unel ${ }^{162}$, Y. Unno ${ }^{65}$, D. Urbaniec ${ }^{34}$, G. Usai ${ }^{7}$, M. Uslenghi ${ }^{118 a, 118 b}$, L. Vacavant ${ }^{82}$, V. Vacek ${ }^{126}$, B. Vachon ${ }^{84}$, S. Vahsen ${ }^{14}$, J. Valenta ${ }^{124}$, P. Valente ${ }^{131 a}$, S. Valentinetti ${ }^{19 a, 19 b}$, S. Valkar ${ }^{125}$, E. Valladolid Gallego ${ }^{166}$, S. Vallecorsa ${ }^{151}$, J.A. Valls Ferrer ${ }^{166}$, H. van der Graaf ${ }^{104}$, E. van der Kraaij ${ }^{104}$, R. Van Der Leeuw ${ }^{104}$, E. van der Poel ${ }^{104}$, D. van der $\operatorname{Ster}^{29}$, N. van Eldik ${ }^{83}$, P. van Gemmeren ${ }^{5}$, Z. van Kesteren ${ }^{104}$, I. van Vulpen ${ }^{104}$, M. Vanadia ${ }^{98}$, W. Vandellii ${ }^{29}$, G. Vandoni ${ }^{29}$, A. Vaniachine ${ }^{5}$, P. Vankov ${ }^{41}$, F. Vannucci ${ }^{77}$, F. Varela Rodriguez ${ }^{29}$, R. Vari ${ }^{131 a}$, E.W. Varnes ${ }^{6}$, D. Varouchas $^{14}$, A. Vartapetian ${ }^{7}$, K.E. Varvell ${ }^{149}$, V.I. Vassilakopoulos ${ }^{56}$, F. Vazeille ${ }^{33}$, T. Vazquez Schroeder ${ }^{54}$, G. Vegni ${ }^{88 a, 88 b}$, J.J. Veillet ${ }^{114}$, C. Vellidis ${ }^{8}$, F. Veloso ${ }^{123 a}$, R. Veness ${ }^{29}$, S. Veneziano ${ }^{131 a}$, A. Ventura ${ }^{71 a, 71 b}$, D. Ventura ${ }^{137}$, M. Venturi ${ }^{48}$, N. Venturi ${ }^{157}$, V. Vercesi ${ }^{118 a}$, M. Verducci ${ }^{137}$, W. Verkerke ${ }^{104}$, J.C. Vermeulen ${ }^{104}$, A. Vest ${ }^{43}$, M.C. Vetterli ${ }^{141, d}$, I. Vichou ${ }^{164}$, T. Vickey ${ }^{144 b, a f}$, O.E. Vickey Boeriu ${ }^{144 b}$, G.H.A. Viehhauser ${ }^{117}$, S. Viel $^{167}$, M. Villa ${ }^{19 a}, 19 b$, M. Villaplana Perez ${ }^{166}$, E. Vilucchi ${ }^{47}$, M.G. Vincter ${ }^{28}$, E. Vinek ${ }^{29}$, V.B. Vinogradov ${ }^{64}$, M. Virchaux ${ }^{135, *}$, J. Virzi ${ }^{14}$, O. Vitells ${ }^{170}$, M. Viti ${ }^{41}$, I. Vivarelli ${ }^{48}$, F. Vives Vaque ${ }^{2}$,

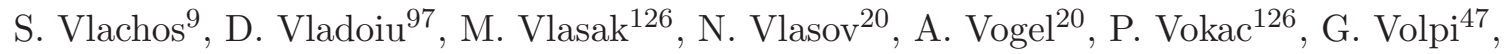


M. Volpi ${ }^{85}$, G. Volpini ${ }^{88 a}$, H. von der Schmitt $^{98}$, J. von Loeben ${ }^{98}$, H. von Radziewski ${ }^{48}$,

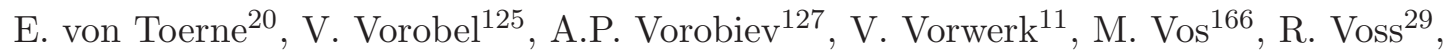
T.T. Voss ${ }^{173}$, J.H. Vossebeld ${ }^{72}$, N. Vranjes ${ }^{135}$, M. Vranjes Milosavljevic ${ }^{104}$, V. Vrba ${ }^{124}$, M. Vreeswijk ${ }^{104}$, T. Vu Anh ${ }^{48}$, R. Vuillermet ${ }^{29}$, I. Vukotic ${ }^{114}$, W. Wagner ${ }^{173}$, P. Wagner ${ }^{119}$, H. Wahlen ${ }^{173}$, J. Wakabayashi ${ }^{100}$, J. Walbersloh ${ }^{42}$, S. Walch ${ }^{86}$, J. Walder ${ }^{70}$, R. Walker ${ }^{97}$, W. Walkowiak ${ }^{140}$, R. Wall ${ }^{174}$, P. Waller ${ }^{72}$, C. Wang ${ }^{44}$, H. Wang ${ }^{171}$, H. Wang ${ }^{32 b, a g}$, J. Wang ${ }^{150}$, J. Wang ${ }^{55}$, J.C. Wang ${ }^{137}$, R. Wang ${ }^{102}$, S.M. Wang ${ }^{150}$, A. Warburton ${ }^{84}$, C.P. Ward ${ }^{27}$, M. Warsinsky ${ }^{48}$, P.M. Watkins ${ }^{17}$, A.T. Watson ${ }^{17}$, I.J. Watson ${ }^{149}$, M.F. Watson ${ }^{17}$, G. Watts ${ }^{137}$, S. Watts ${ }^{81}$, A.T. Waugh ${ }^{149}$, B.M. Waugh ${ }^{76}$, M. Weber ${ }^{128}$, M.S. Weber ${ }^{16}$, P. Weber ${ }^{54}$, A.R. Weidberg ${ }^{117}$, P. Weigell ${ }^{98}$, J. Weingarten ${ }^{54}$, C. Weiser ${ }^{48}$, H. Wellenstein ${ }^{22}$, P.S. Wells ${ }^{29}$, T. Wenaus ${ }^{24}$,

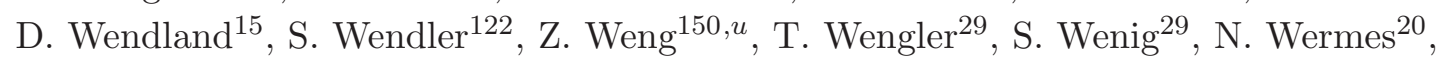
M. Werner ${ }^{48}$, P. Werner ${ }^{29}$, M. Werth ${ }^{162}$, M. Wessels ${ }^{58 a}$, C. Weydert ${ }^{55}$, K. Whalen ${ }^{28}$, S.J. Wheeler-Ellis ${ }^{162}$, S.P. Whitaker ${ }^{21}$, A. White ${ }^{7}$, M.J. White ${ }^{85}$, S.R. Whitehead ${ }^{117}$, D. Whiteson ${ }^{162}$, D. Whittington ${ }^{60}$, F. Wicek ${ }^{114}$, D. Wicke ${ }^{173}$, F.J. Wickens ${ }^{128}$, W. Wiedenmann ${ }^{171}$, M. Wielers ${ }^{128}$, P. Wienemann ${ }^{20}$, C. Wiglesworth ${ }^{74}$, L.A.M. Wiik-Fuchs ${ }^{48}$, P.A. Wijeratne ${ }^{76}$, A. Wildauer ${ }^{166}$, M.A. Wildt ${ }^{41, q}$, I. Wilhelm ${ }^{125}$, H.G. Wilkens ${ }^{29}$, J.Z. Will ${ }^{97}$, E. Williams ${ }^{34}$, H.H. Williams ${ }^{119}$, W. Willis ${ }^{34}$, S. Willocq ${ }^{83}$, J.A. Wilson ${ }^{17}$, M.G. Wilson ${ }^{142}$, A. Wilson ${ }^{86}$, I. Wingerter-Seez ${ }^{4}$, S. Winkelmann ${ }^{48}$, F. Winklmeier ${ }^{29}$, M. Wittgen ${ }^{142}$, M.W. Wolter ${ }^{38}$, H. Wolters ${ }^{123 a}, h$, W.C. Wong ${ }^{40}$, G. Wooden ${ }^{86}$, B.K. Wosiek ${ }^{38}$, J. Wotschack ${ }^{29}$, M.J. Woudstra ${ }^{83}$, K.W. Wozniak ${ }^{38}$, K. Wraight ${ }^{53}$, C. Wright ${ }^{53}$, M. Wright ${ }^{53}$, B. Wrona ${ }^{72}$, S.L. Wu ${ }^{171}$, X. $\mathrm{Wu}^{49}$, Y. Wu ${ }^{32 b, a h}$,

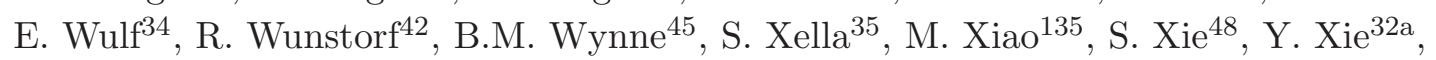

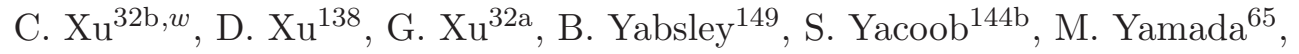
H. Yamaguchi ${ }^{154}$, A. Yamamoto ${ }^{65}$, K. Yamamoto ${ }^{63}$, S. Yamamoto $^{154}$, T. Yamamura $^{154}$, T. Yamanaka ${ }^{154}$, J. Yamaoka ${ }^{44}$, T. Yamazaki ${ }^{154}$, Y. Yamazaki ${ }^{66}$, Z. Yan ${ }^{21}$, H. Yang ${ }^{86}$, U.K. Yang ${ }^{81}$, Y. Yang ${ }^{60}$, Y. Yang ${ }^{32 a}$, Z. Yang ${ }^{145 a, 145 b}$, S. Yanush ${ }^{90}$, Y. Yao ${ }^{14}$, Y. Yasu ${ }^{65}$, G.V. Ybeles Smit ${ }^{129}$, J. Ye ${ }^{39}$, S. Ye ${ }^{24}$, M. Yilmaz ${ }^{3 c}$, R. Yoosoofmiya ${ }^{122}$, K. Yorita ${ }^{169}$, R. Yoshida ${ }^{5}$, C. Young ${ }^{142}$, S. Youssef ${ }^{21}$, D. Yu ${ }^{24}$, J. Yu$^{7}$, J. Yu ${ }^{111}$, L. Yuan ${ }^{32 a, a i}$, A. Yurkewicz ${ }^{105}$, B. Zabinski ${ }^{38}$, V.G. Zaets ${ }^{127}$, R. Zaidan ${ }^{62}$, A.M. Zaitsev ${ }^{127}$, Z. Zajacova ${ }^{29}$, L. Zanello ${ }^{131 a, 131 b}$, A. Zaytsev ${ }^{106}$, C. Zeitnitz ${ }^{173}$, M. Zeller ${ }^{174}$, M. Zeman ${ }^{124}$, A. Zemla ${ }^{38}$, C. Zendler ${ }^{20}$, O. Zenin ${ }^{127}$, T. Ženišs ${ }^{143 a}$, Z. Zinonos ${ }^{121 a, 121 b}$, S. Zenz ${ }^{14}$, D. Zerwas ${ }^{114}$, G. Zevi della Porta ${ }^{57}$, Z. Zhan ${ }^{32 d}$, D. Zhang ${ }^{32 b, a g}$, H. Zhang ${ }^{87}$, J. Zhang ${ }^{5}$, X. Zhang ${ }^{32 d}$, Z. Zhang ${ }^{114}$, L. Zhao ${ }^{107}$, T. Zhao ${ }^{137}$, Z. Zhao ${ }^{32 b}$,

A. Zhemchugov ${ }^{64}$, S. Zheng ${ }^{32 a}$, J. Zhong ${ }^{117}$, B. Zhou ${ }^{86}$, N. Zhou ${ }^{162}$, Y. Zhou ${ }^{150}$, C.G. Zhu ${ }^{32 \mathrm{~d}}$, H. Zhu ${ }^{41}$, J. Zhu ${ }^{86}$, Y. Zhu ${ }^{32 \mathrm{~b}}$, X. Zhuang ${ }^{97}$, V. Zhuravlov ${ }^{98}$, D. Zieminska ${ }^{60}$, R. Zimmermann ${ }^{20}$, S. Zimmermann ${ }^{20}$, S. Zimmermann ${ }^{48}$, M. Ziolkowski140, R. Zitoun ${ }^{4}$, L. Živkovic ${ }^{34}$, V.V. Zmouchko ${ }^{127, *}$, G. Zobernig ${ }^{171}$, A. Zoccoli ${ }^{19 a, 19 b}$, Y. Zolnierowski ${ }^{4}$, A. Zsenei ${ }^{29}$, M. zur Nedden ${ }^{15}$, V. Zutshi ${ }^{105}$, L. Zwalinski ${ }^{29}$ 
${ }^{1}$ University at Albany, Albany NY, U.S.A.

${ }^{2}$ Department of Physics, University of Alberta, Edmonton AB, Canada

$3{ }^{(a)}$ Department of Physics, Ankara University, Ankara, Turkey

${ }^{(b)}$ Department of Physics, Dumlupinar University, Kutahya, Turkey

(c) Department of Physics, Gazi University, Ankara, Turkey

(d) Division of Physics, TOBB University of Economics and Technology, Ankara, Turkey

${ }^{(e)}$ Turkish Atomic Energy Authority, Ankara, Turkey

${ }^{4}$ LAPP, CNRS/IN2P3 and Université de Savoie, Annecy-le-Vieux, France

${ }^{5}$ High Energy Physics Division, Argonne National Laboratory, Argonne IL, U.S.A.

${ }^{6}$ Department of Physics, University of Arizona, Tucson AZ, U.S.A.

${ }^{7}$ Department of Physics, The University of Texas at Arlington, Arlington TX, U.S.A.

8 Physics Department, University of Athens, Athens, Greece

${ }^{9}$ Physics Department, National Technical University of Athens, Zografou, Greece

${ }^{10}$ Institute of Physics, Azerbaijan Academy of Sciences, Baku, Azerbaijan

${ }^{11}$ Institut de Física d'Altes Energies and Departament de Física de la

Universitat Autònoma de Barcelona and ICREA, Barcelona, Spain

12 (a) Institute of Physics, University of Belgrade, Belgrade, Serbia

${ }^{(b)}$ Vinca Institute of Nuclear Sciences, University of Belgrade, Belgrade, Serbia

${ }^{13}$ Department for Physics and Technology, University of Bergen, Bergen, Norway

14 Physics Division, Lawrence Berkeley National Laboratory and University of California, Berkeley CA, U.S.A.

${ }^{15}$ Department of Physics, Humboldt University, Berlin, Germany

16 Albert Einstein Center for Fundamental Physics and Laboratory for High Energy Physics, University of Bern, Bern, Switzerland

17 School of Physics and Astronomy, University of Birmingham, Birmingham, U.K.

$18{ }^{(a)}$ Department of Physics, Bogazici University, Istanbul, Turkey

${ }^{(b)}$ Division of Physics, Dogus University, Istanbul, Turkey

${ }^{(c)}$ Department of Physics Engineering, Gaziantep University, Gaziantep, Turkey

${ }^{(d)}$ Department of Physics, Istanbul Technical University, Istanbul, Turkey

$19{ }^{(a)}$ INFN Sezione di Bologna, Italy

${ }^{(b)}$ Dipartimento di Fisica, Università di Bologna, Bologna, Italy

${ }^{20}$ Physikalisches Institut, University of Bonn, Bonn, Germany

${ }^{21}$ Department of Physics, Boston University, Boston MA, U.S.A.

22 Department of Physics, Brandeis University, Waltham MA, U.S.A.

$23{ }^{(a)}$ Universidade Federal do Rio De Janeiro COPPE/EE/IF, Rio de Janeiro, Brazil

${ }^{(b)}$ Federal University of Juiz de Fora (UFJF), Juiz de Fora, Brazil

${ }^{(c)}$ Federal University of Sao Joao del Rei (UFSJ), Sao Joao del Rei, Brazil

${ }^{(d)}$ Instituto de Fisica, Universidade de Sao Paulo, Sao Paulo, Brazil

${ }^{24}$ Physics Department, Brookhaven National Laboratory, Upton NY, U.S.A.

$25{ }^{(a)}$ National Institute of Physics and Nuclear Engineering, Bucharest, Romania

${ }^{(b)}$ University Politehnica Bucharest, Bucharest; ${ }^{(c)}$ West University in Timisoara, Timisoara, Romania

${ }^{26}$ Departamento de Física, Universidad de Buenos Aires, Buenos Aires, Argentina 
27 Cavendish Laboratory, University of Cambridge, Cambridge, U.K.

28 Department of Physics, Carleton University, Ottawa ON, Canada

${ }^{29}$ CERN, Geneva, Switzerland

${ }^{30}$ Enrico Fermi Institute, University of Chicago, Chicago IL, U.S.A.

$31{ }^{(a)}$ Departamento de Fisica, Pontificia Universidad Católica de Chile, Santiago, Chile

${ }^{(b)}$ Departamento de Física, Universidad Técnica Federico Santa María, Valparaíso, Chile

$32{ }^{(a)}$ Institute of High Energy Physics, Chinese Academy of Sciences, Beijing, China

${ }^{(b)}$ Department of Modern Physics, University of Science and Technology of China, Anhui, China

${ }^{(c)}$ Department of Physics, Nanjing University, Jiangsu, China

${ }^{(d)}$ School of Physics, Shandong University, Shandong, China

${ }^{33}$ Laboratoire de Physique Corpusculaire, Clermont Université and

Université Blaise Pascal and CNRS/IN2P3, Aubiere Cedex, France

${ }^{34}$ Nevis Laboratory, Columbia University, Irvington NY, U.S.A.

35 Niels Bohr Institute, University of Copenhagen, Kobenhavn, Denmark

$36{ }^{(a)}$ INFN Gruppo Collegato di Cosenza, Italy

${ }^{(b)}$ Dipartimento di Fisica, Università della Calabria, Arcavata di Rende, Italy

${ }^{37}$ AGH University of Science and Technology, Faculty of Physics and Applied Computer Science, Krakow, Poland

38 The Henryk Niewodniczanski Institute of Nuclear Physics,

Polish Academy of Sciences, Krakow, Poland

39 Physics Department, Southern Methodist University, Dallas TX, U.S.A.

${ }^{40}$ Physics Department, University of Texas at Dallas, Richardson TX, U.S.A.

${ }^{41}$ DESY, Hamburg and Zeuthen, Germany

${ }^{42}$ Institut für Experimentelle Physik IV, Technische Universität Dortmund, Dortmund, Germany

${ }^{43}$ Institut für Kern- und Teilchenphysik, Technical University Dresden, Dresden, Germany

${ }^{44}$ Department of Physics, Duke University, Durham NC, U.S.A.

${ }^{45}$ SUPA - School of Physics and Astronomy, University of Edinburgh, Edinburgh, U.K.

${ }^{46}$ Fachhochschule Wiener Neustadt, Johannes Gutenbergstrasse 32700 Wiener Neustadt, Austria

${ }^{47}$ INFN Laboratori Nazionali di Frascati, Frascati, Italy

${ }^{48}$ Fakultät für Mathematik und Physik, Albert-Ludwigs-Universität, Freiburg i.Br., Germany

49 Section de Physique, Université de Genève, Geneva, Switzerland

$50{ }^{(a)}$ INFN Sezione di Genova, Italy

${ }^{(b)}$ Dipartimento di Fisica, Università di Genova, Genova, Italy

$51{ }^{(a)}$ E.Andronikashvili Institute of Physics, Tbilisi State University, Tbilisi, Georgia

${ }^{(b)}$ High Energy Physics Institute, Tbilisi State University, Tbilisi, Georgia

${ }^{52}$ II Physikalisches Institut, Justus-Liebig-Universität Giessen, Giessen, Germany

53 SUPA - School of Physics and Astronomy, University of Glasgow, Glasgow, U.K. 
54 II Physikalisches Institut, Georg-August-Universität, Göttingen, Germany

${ }^{55}$ Laboratoire de Physique Subatomique et de Cosmologie, Université Joseph Fourier and CNRS/IN2P3 and Institut National Polytechnique de Grenoble, Grenoble, France

${ }^{56}$ Department of Physics, Hampton University, Hampton VA, U.S.A.

${ }^{57}$ Laboratory for Particle Physics and Cosmology, Harvard University, Cambridge MA, U.S.A.

58 (a) Kirchhoff-Institut für Physik, Ruprecht-Karls-Universität Heidelberg, Heidelberg, Germany

(b) Physikalisches Institut, Ruprecht-Karls-Universität Heidelberg, Heidelberg, Germany

${ }^{(c)}$ ZITI Institut für technische Informatik, Ruprecht-Karls-Universität Heidelberg, Mannheim, Germany

${ }^{59}$ Faculty of Applied Information Science, Hiroshima Institute of Technology, Hiroshima, Japan

${ }^{60}$ Department of Physics, Indiana University, Bloomington IN, U.S.A.

${ }^{61}$ Institut für Astro- und Teilchenphysik, Leopold-Franzens-Universität, Innsbruck, Austria

${ }^{62}$ University of Iowa, Iowa City IA, U.S.A.

63 Department of Physics and Astronomy, Iowa State University, Ames IA, U.S.A.

${ }^{64}$ Joint Institute for Nuclear Research, JINR Dubna, Dubna, Russia

${ }^{65}$ KEK, High Energy Accelerator Research Organization, Tsukuba, Japan

${ }^{66}$ Graduate School of Science, Kobe University, Kobe, Japan

${ }^{67}$ Faculty of Science, Kyoto University, Kyoto, Japan

${ }^{68}$ Kyoto University of Education, Kyoto, Japan

${ }^{69}$ Instituto de Física La Plata, Universidad Nacional de La Plata and CONICET, La Plata, Argentina

70 Physics Department, Lancaster University, Lancaster, U.K.

$71{ }^{(a)}$ INFN Sezione di Lecce, Italy

${ }^{(b)}$ Dipartimento di Fisica, Università del Salento, Lecce, Italy

72 Oliver Lodge Laboratory, University of Liverpool, Liverpool, U.K.

${ }^{73}$ Department of Physics, Jožef Stefan Institute and University of Ljubljana, Ljubljana, Slovenia

${ }^{74}$ School of Physics and Astronomy, Queen Mary University of London, London,U.K.

75 Department of Physics, Royal Holloway University of London, Surrey, U.K.

${ }^{76}$ Department of Physics and Astronomy, University College London, London, U.K.

77 Laboratoire de Physique Nucléaire et de Hautes Energies, UPMC and Université Paris-Diderot and CNRS/IN2P3, Paris, France

78 Fysiska institutionen, Lunds universitet, Lund, Sweden

${ }^{79}$ Departamento de Fisica Teorica C-15, Universidad Autonoma de Madrid, Madrid, Spain

${ }^{80}$ Institut für Physik, Universität Mainz, Mainz, Germany

81 School of Physics and Astronomy, University of Manchester, Manchester, U.K.

${ }^{82}$ CPPM, Aix-Marseille Université and CNRS/IN2P3, Marseille, France

83 Department of Physics, University of Massachusetts, Amherst MA, U.S.A. 
84 Department of Physics, McGill University, Montreal QC, Canada

${ }^{85}$ School of Physics, University of Melbourne, Victoria, Australia

${ }^{86}$ Department of Physics, The University of Michigan, Ann Arbor MI, U.S.A.

${ }^{87}$ Department of Physics and Astronomy, Michigan State University, East Lansing MI, U.S.A.

88 (a) $^{(b N F N ~ S e z i o n e ~ d i ~ M i l a n o, ~ I t a l y ~}$

(b) Dipartimento di Fisica, Università di Milano, Milano, Italy

89 B.I. Stepanov Institute of Physics, National Academy of Sciences of Belarus, Minsk, Republic of Belarus

90 National Scientific and Educational Centre for Particle and High Energy Physics, Minsk, Republic of Belarus

${ }^{91}$ Department of Physics, Massachusetts Institute of Technology, Cambridge MA, U.S.A.

92 Group of Particle Physics, University of Montreal, Montreal QC, Canada

${ }^{93}$ P.N. Lebedev Institute of Physics, Academy of Sciences, Moscow, Russia

${ }^{94}$ Institute for Theoretical and Experimental Physics (ITEP), Moscow, Russia

95 Moscow Engineering and Physics Institute (MEPhI), Moscow, Russia

96 Skobeltsyn Institute of Nuclear Physics, Lomonosov Moscow State University, Moscow, Russia

${ }^{97}$ Fakultät für Physik, Ludwig-Maximilians-Universität München, München, Germany

98 Max-Planck-Institut für Physik (Werner-Heisenberg-Institut), München, Germany

99 Nagasaki Institute of Applied Science, Nagasaki, Japan

100 Graduate School of Science, Nagoya University, Nagoya, Japan

$101{ }^{(a)}$ INFN Sezione di Napoli, Italy

${ }^{(b)}$ Dipartimento di Scienze Fisiche, Università di Napoli, Napoli, Italy

102 Department of Physics and Astronomy, University of New Mexico, Albuquerque NM, U.S.A.

103 Institute for Mathematics, Astrophysics and Particle Physics, Radboud University Nijmegen/Nikhef, Nijmegen, Netherlands

104 Nikhef National Institute for Subatomic Physics and University of Amsterdam, Amsterdam, Netherlands

105 Department of Physics, Northern Illinois University, DeKalb IL, U.S.A.

106 Budker Institute of Nuclear Physics, SB RAS, Novosibirsk, Russia

107 Department of Physics, New York University, New York NY, U.S.A.

108 Ohio State University, Columbus OH, U.S.A.

109 Faculty of Science, Okayama University, Okayama, Japan

110 Homer L. Dodge Department of Physics and Astronomy, University of Oklahoma, Norman OK, U.S.A.

111 Department of Physics, Oklahoma State University, Stillwater OK, U.S.A.

112 Palacký University, RCPTM, Olomouc, Czech Republic

113 Center for High Energy Physics, University of Oregon, Eugene OR, U.S.A.

${ }^{114}$ LAL, Univ. Paris-Sud and CNRS/IN2P3, Orsay, France

115 Graduate School of Science, Osaka University, Osaka, Japan

116 Department of Physics, University of Oslo, Oslo, Norway

117 Department of Physics, Oxford University, Oxford, U.K. 
$118{ }^{(a)}$ INFN Sezione di Pavia, Italy

${ }^{(b)}$ Dipartimento di Fisica, Università di Pavia, Pavia, Italy

119 Department of Physics, University of Pennsylvania, Philadelphia PA, U.S.A.

120 Petersburg Nuclear Physics Institute, Gatchina, Russia

$121{ }^{(a)}$ INFN Sezione di Pisa, Italy

${ }^{(b)}$ Dipartimento di Fisica E. Fermi, Università di Pisa, Pisa, Italy

${ }^{122}$ Department of Physics and Astronomy, University of Pittsburgh, Pittsburgh PA, U.S.A.

$123{ }^{(a)}$ Laboratorio de Instrumentacao e Fisica Experimental de Particulas - LIP, Lisboa, Portugal

${ }^{(b)}$ Departamento de Fisica Teorica y del Cosmos and CAFPE, Universidad de Granada, Granada, Spain

124 Institute of Physics, Academy of Sciences of the Czech Republic, Praha,

Czech Republic

${ }^{125}$ Faculty of Mathematics and Physics, Charles University in Prague, Praha, Czech Republic

${ }^{126}$ Czech Technical University in Prague, Praha, Czech Republic

127 State Research Center Institute for High Energy Physics, Protvino, Russia

128 Particle Physics Department, Rutherford Appleton Laboratory, Didcot, U.K.

129 Physics Department, University of Regina, Regina SK, Canada

130 Ritsumeikan University, Kusatsu, Shiga, Japan

$131{ }^{(a)}$ INFN Sezione di Roma I, Italy

${ }^{(b)}$ Dipartimento di Fisica, Università La Sapienza, Roma, Italy

$132{ }^{(a)}$ INFN Sezione di Roma Tor Vergata, Italy

${ }^{(b)}$ Dipartimento di Fisica, Università di Roma Tor Vergata, Roma, Italy

$133{ }^{(a)}$ INFN Sezione di Roma Tre, Italy

(b) Dipartimento di Fisica, Università Roma Tre, Roma, Italy

$134{ }^{(a)}$ Faculté des Sciences Ain Chock, Réseau Universitaire de Physique des Hautes Energies - Université Hassan II, Casablanca, Morocco

${ }^{(b)}$ Centre National de l'Energie des Sciences Techniques Nucleaires, Rabat, Morocco

${ }^{(c)}$ Faculté des Sciences Semlalia, Université Cadi Ayyad, LPHEA-Marrakech , Morocco

${ }^{(d)}$ Faculté des Sciences, Université Mohamed Premier and LPTPM, Oujda, Morocco

${ }^{(e)}$ Faculté des Sciences, Université Mohammed V- Agdal, Rabat, Morocco

135 DSM/IRFU (Institut de Recherches sur les Lois Fondamentales de l'Univers),

CEA Saclay (Commissariat a l'Energie Atomique), Gif-sur-Yvette, France

136 Santa Cruz Institute for Particle Physics, University of California Santa Cruz, Santa Cruz CA, U.S.A.

137 Department of Physics, University of Washington, Seattle WA, U.S.A.

138 Department of Physics and Astronomy, University of Sheffield, Sheffield, U.K.

139 Department of Physics, Shinshu University, Nagano, Japan

140 Fachbereich Physik, Universität Siegen, Siegen, Germany

141 Department of Physics, Simon Fraser University, Burnaby BC, Canada 
142 SLAC National Accelerator Laboratory, Stanford CA, U.S.A.

$143{ }^{(a)}$ Faculty of Mathematics, Physics \& Informatics, Comenius University, Bratislava, Slovak Republic

${ }^{(b)}$ Department of Subnuclear Physics, Institute of Experimental Physics of the Slovak Academy of Sciences, Kosice, Slovak Republic

144 (a) Department of Physics, University of Johannesburg, Johannesburg, South Africa

${ }^{(b)}$ School of Physics, University of the Witwatersrand, Johannesburg, South Africa

$145{ }^{(a)}$ Department of Physics, Stockholm University, Sweden

(b) The Oskar Klein Centre, Stockholm, Sweden

146 Physics Department, Royal Institute of Technology, Stockholm, Sweden

147 Departments of Physics \& Astronomy and Chemistry, Stony Brook University, Stony Brook NY, U.S.A.

148 Department of Physics and Astronomy, University of Sussex, Brighton, U.K.

149 School of Physics, University of Sydney, Sydney, Australia

${ }^{150}$ Institute of Physics, Academia Sinica, Taipei, Taiwan

${ }^{151}$ Department of Physics, Technion: Israel Inst. of Technology, Haifa, Israel

152 Raymond and Beverly Sackler School of Physics and Astronomy, Tel Aviv University, Tel Aviv, Israel

153 Department of Physics, Aristotle University of Thessaloniki, Thessaloniki, Greece

${ }^{154}$ International Center for Elementary Particle Physics and Department of Physics, The University of Tokyo, Tokyo, Japan

155 Graduate School of Science and Technology, Tokyo Metropolitan University, Tokyo, Japan

156 Department of Physics, Tokyo Institute of Technology, Tokyo, Japan

157 Department of Physics, University of Toronto, Toronto ON, Canada

$158{ }^{(a)}$ TRIUMF, Vancouver BC, Canada

(b) Department of Physics and Astronomy, York University, Toronto ON, Canada

${ }^{159}$ Institute of Pure and Applied Sciences, University of Tsukuba,1-1-1 Tennodai, Tsukuba, Ibaraki 305-8571, Japan

160 Science and Technology Center, Tufts University, Medford MA, U.S.A.

161 Centro de Investigaciones, Universidad Antonio Narino, Bogota, Colombia

162 Department of Physics and Astronomy, University of California Irvine, Irvine CA, U.S.A.

$163{ }^{(a)}$ INFN Gruppo Collegato di Udine, Italy

(b) ICTP, Trieste, Italy

${ }^{(c)}$ Dipartimento di Chimica, Fisica e Ambiente, Università di Udine, Udine, Italy

164 Department of Physics, University of Illinois, Urbana IL, U.S.A.

165 Department of Physics and Astronomy, University of Uppsala, Uppsala, Sweden

166 Instituto de Física Corpuscular (IFIC) and Departamento de Física Atómica, Molecular y Nuclear and Departamento de Ingeniería Electrónica and

Instituto de Microelectrónica de Barcelona (IMB-CNM), University of Valencia and CSIC, Valencia, Spain

167 Department of Physics, University of British Columbia, Vancouver BC, Canada 
168 Department of Physics and Astronomy, University of Victoria, Victoria BC, Canada 169 Waseda University, Tokyo, Japan

170 Department of Particle Physics, The Weizmann Institute of Science, Rehovot, Israel

171 Department of Physics, University of Wisconsin, Madison WI, U.S.A.

172 Fakultät für Physik und Astronomie, Julius-Maximilians-Universität, Würzburg, Germany

${ }^{173}$ Fachbereich C Physik, Bergische Universität Wuppertal, Wuppertal, Germany

174 Department of Physics, Yale University, New Haven CT, U.S.A.

175 Yerevan Physics Institute, Yerevan, Armenia

176 Domaine scientifique de la Doua, Centre de Calcul CNRS/IN2P3, Villeurbanne Cedex, France

177 Faculty of Science, Hiroshima University, Hiroshima, Japan

${ }^{a}$ Also at Laboratorio de Instrumentacao e Fisica Experimental de Particulas - LIP, Lisboa, Portugal

${ }^{b}$ Also at Faculdade de Ciencias and CFNUL, Universidade de Lisboa, Lisboa, Portugal

${ }^{c}$ Also at Particle Physics Department, Rutherford Appleton Laboratory, Didcot, U.K.

${ }^{d}$ Also at TRIUMF, Vancouver BC, Canada

${ }^{e}$ Also at Department of Physics, California State University, Fresno CA, U.S.A.

${ }^{f}$ Also at Novosibirsk State University, Novosibirsk, Russia

${ }^{g}$ Also at Fermilab, Batavia IL, U.S.A.

${ }^{h}$ Also at Department of Physics, University of Coimbra, Coimbra, Portugal

${ }^{i}$ Also at Università di Napoli Parthenope, Napoli, Italy

$j$ Also at Institute of Particle Physics (IPP), Canada

${ }^{k}$ Also at Department of Physics, Middle East Technical University, Ankara, Turkey

${ }^{l}$ Also at Louisiana Tech University, Ruston LA, U.S.A.

$m$ Also at Department of Physics and Astronomy, University College London, London, U.K.

${ }^{n}$ Also at Group of Particle Physics, University of Montreal, Montreal QC, Canada

${ }^{o}$ Also at Department of Physics, University of Cape Town, Cape Town, South Africa

${ }^{p}$ Also at Institute of Physics, Azerbaijan Academy of Sciences, Baku, Azerbaijan

${ }^{q}$ Also at Institut für Experimentalphysik, Universität Hamburg, Hamburg, Germany

${ }^{r}$ Also at Manhattan College, New York NY, U.S.A.

$s$ Also at School of Physics, Shandong University, Shandong, China

${ }^{t}$ Also at CPPM, Aix-Marseille Université and CNRS/IN2P3, Marseille, France

${ }^{u}$ Also at School of Physics and Engineering, Sun Yat-sen University, Guanzhou, China

$v$ Also at Academia Sinica Grid Computing, Institute of Physics, Academia Sinica, Taipei, Taiwan

${ }^{w}$ Also at DSM/IRFU (Institut de Recherches sur les Lois Fondamentales de l'Univers), CEA Saclay (Commissariat a l'Energie Atomique), Gif-sur-Yvette, France

${ }^{x}$ Also at Section de Physique, Université de Genève, Geneva, Switzerland

${ }^{y}$ Also at Departamento de Fisica, Universidade de Minho, Braga, Portugal 
${ }^{z}$ Also at Department of Physics and Astronomy, University of South Carolina, Columbia SC, U.S.A.

aa Also at Institute for Particle and Nuclear Physics, Wigner Research Centre for Physics, Budapest, Hungary

${ }^{a b}$ Also at California Institute of Technology, Pasadena CA, U.S.A.

ac Also at Institute of Physics, Jagiellonian University, Krakow, Poland

ad Also at LAL, Univ. Paris-Sud and CNRS/IN2P3, Orsay, France

ae Also at Department of Physics and Astronomy, University of Sheffield, Sheffield, U.K.

af Also at Department of Physics, Oxford University, Oxford, U.K.

ag Also at Institute of Physics, Academia Sinica, Taipei, Taiwan

ah Also at Department of Physics, The University of Michigan, Ann Arbor MI, U.S.A.

${ }^{a i}$ Also at Laboratoire de Physique Nucléaire et de Hautes Energies, UPMC and Université Paris-Diderot and CNRS/IN2P3, Paris, France

* Deceased 